\title{
Hostility and vital exhaustion as risk indicators of myocardial infarction
}

\author{
Citation for published version (APA):
}

Meesters, C. M. G. (1995). Hostility and vital exhaustion as risk indicators of myocardial infarction. [Doctoral Thesis, Maastricht University]. Datawyse / Universitaire Pers Maastricht. https://doi.org/10.26481/dis. $19951214 \mathrm{~cm}$

Document status and date:

Published: 01/01/1995

DOI:

$10.26481 /$ dis. $19951214 \mathrm{~cm}$

Document Version:

Publisher's PDF, also known as Version of record

\section{Please check the document version of this publication:}

- A submitted manuscript is the version of the article upon submission and before peer-review. There can be important differences between the submitted version and the official published version of record.

People interested in the research are advised to contact the author for the final version of the publication, or visit the DOI to the publisher's website.

- The final author version and the galley proof are versions of the publication after peer review.

- The final published version features the final layout of the paper including the volume, issue and page numbers.

Link to publication

\footnotetext{
General rights rights.

- You may freely distribute the URL identifying the publication in the public portal. please follow below link for the End User Agreement:

www.umlib.nl/taverne-license

Take down policy

If you believe that this document breaches copyright please contact us at:

repository@maastrichtuniversity.nl

providing details and we will investigate your claim.
}

Copyright and moral rights for the publications made accessible in the public portal are retained by the authors and/or other copyright owners and it is a condition of accessing publications that users recognise and abide by the legal requirements associated with these

- Users may download and print one copy of any publication from the public portal for the purpose of private study or research.

- You may not further distribute the material or use it for any profit-making activity or commercial gain

If the publication is distributed under the terms of Article $25 \mathrm{fa}$ of the Dutch Copyright Act, indicated by the "Taverne" license above, 


\section{HOSTILITY AND VITAL EXHAUSTION AS RISK INDICATORS OF MYOCARDIAL INFARCTION}


Lay-out:

Isel van Noppen

Druk:

Datawyse / Universitaire Pers Maastricht

CIP-DATA KONINKLIJKE BIBLIOTHEEK, DEN HAAG

Meesters, Cornelis Martinus Gerardus

Hostilicy and vital exhaustion as risk indicators of myocandial infarction / Conelis Martinus Gerardus Meesters. - Madstricht : Universitaire Pers Maastricht Thesis Rijksuniversiteit Limburg Mastricht. - With ref. With a summary in Dutch.

ISBN 90-5278-203-2.

Subject headings: hostility and myocardial infarction / vital exhaustion and myocardial infarction. 


\section{HOSTILITY AND VITAL EXHAUSTION AS RISK INDICATORS OF MYOCARDIAL INFARCTION}

\section{PROEFSCHRIFT}

ter verkrijging van de graad van doctor aan de Rijksuniversiteit Limburg te Maastricht, op gezag van de Rector Magnificus, Prof.Mr. M.J. Cohen, ingevolge het besluit van het College van Dekanen, in het openbaar te verdedigen op donderdag 14 december 1995 om 16.00 uur

door

Cornelis Martinus Gerardus Meesters 


\section{Promotor:}

Prof.dr. A.P.W.M. Appels

\section{Beoordelingscommissie:}

Prof.dr. M.A. wan den Hout (voorzitter)

Dr. F.W.H.M. Bär

Dr. J.P.M. Diederiks

Prof.dr. P.G. Heymans (Universiteit Utrecht)

Prof.dr. J.W. van Ree

Financial support by the Netherlands Heart Foundation for the publication of this thesis is gratefully acknowledged. 


\section{CONTENTS}

Chapter 1. Introduction 9

1.1 Hostility and health: an overview 9

1.1.1 From Type A to hostility: the story of coronary prone behavior 10

$\begin{array}{ll}1.1 .2 \text { Conceptual definitions. } & 12\end{array}$

$\begin{array}{ll}1.1 .3 \text { The assessment of hostility } & 14\end{array}$

1.1.4 Hostility and health: evidence of an association 20

1.1.5 Potential mechanisms linking hostility and health 38

1.1.6 Developmental antecedents of hostility 41

1.2 Vital exhaustion and coronary heart disease 43

1.2.1 Vital exhaustion as a precursor of myocardial infarction 44

1.2.2 Evidence from prospective studies 44

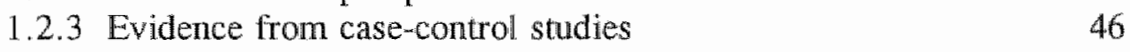

1.2.4 Vital exhaustion and depression 47

1.2.5 Vital exhaustion: a marker of subclinical heart disease? 48

$\begin{array}{ll}1.2 .6 \text { Conclusion } & 48\end{array}$

1.3 Design of the thesis 49

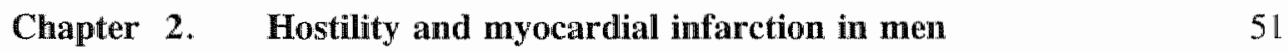

2.1 Summary 51

2.2 Introduction 51

2.3 Method 53

2.4 Results 55

2.5 Discussion $\quad 57$

Chapter 3. Dimensions of hostility and myocardial infarction in adult males

3.1 Summary 59

3.2 Introduction $\quad 59$

3.3 Method 61

3.4 Results 62

3.5 Discussion $\quad 64$ 
Chapter 4. Hostility and perceived parental rearing behaviour

4.1 Summary 67

4.2 Introduction 67

4.3 Method 68

4.4 Results and discussion $\quad 69$

Chapter 5. The relationship between hostility and perceived parental rearing behaviour: a study of male myocardial infarction patients and healthy controls

5.1 Surmmary

$\begin{array}{lll}5.2 & \text { Introduction } & 75\end{array}$

$\begin{array}{lll}5.3 \text { Method } & 77\end{array}$

5.4 Results $\quad 80$

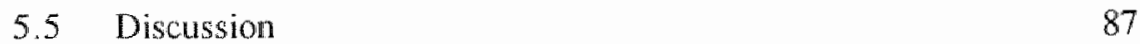

Chapter 6. An interview to measure vital exhaustion.

I. Development and comparison with the Maastricht Questionnaire

6.1 Summary $\quad 89$

6.2 Introduction $\quad 89$

6.3 Construction of the interview, format and scoring 94

6.4 The occurrence of exhaustion before myocardial infarction: empirical data 95

6.5 Correspondence between the interview and the questionnaire 97

6.6 Discriminating power of the questionnaire and the interview 98

$\begin{array}{lll}6.7 & \text { Discussion } & 98\end{array}$

Chapter 7. An interview to measure vital exhaustion.

II. Reliability and validity of the interview and correlations of vital exhaustion with personality characteristics

7.1 Summary 107

$\begin{array}{lll}7.2 & \text { Introduction } & 107\end{array}$

$\begin{array}{lll}7.3 \text { Method } & 108\end{array}$

$\begin{array}{lll}7.4 & \text { Results } & 110\end{array}$

$\begin{array}{ll}7.5 \text { Discussion } & 114\end{array}$ 
Chapter 8. Hostility, vital exhaustion and myocardial infarction: discriminating power and interrelationships

8.1 Summary

8.2 Introduction

8.3 Method

8.4 Results

8.5 Discussion

Chapter 9. General discussion

9.1 Relationship between hostility and myocardial infarction

9.2 Developmental aspects of hostility

9.3 Vital exhaustion and myocardial infarction: the development of an interview

9.4 Hostility, vital exhaustion and myocardial infarction

9.5 Methodological limitations

9.6 Conclusions

9.7 Implications for future research and suggestions for prevention and intervention

References

Summary

Samenvatting

Appendix

List of publications

Dankwoord 
But, at the last, what do we know? Arthur Machen (1863-1947) 


\section{Chapter 1}

\section{INTRODUCTION}

This introductory chapter starts with an extensive essay on the association between hostility and health (section 1.1), and in particular on the relationship between hostility and coronary heart disease. The second section provides an overview of the premonitory symptoms of myocardial infarction (MI) and sudden cardiac death (SCD) (section 1.2). Finally, section 1.3 gives a brief outline of the present thesis.

\subsection{HOSTILITY AND HEALTH: AN OVERVIEW}

Recent developments in the fields of behavioral medicine and health psychology have led to a revival of the old psychosomatic hypothesis that hostility and anger play a role in the etiology of coronary heart disease (CHD) and other serious diseases. The past fifteen years have witnessed the publication of a wealth of studies of hostility and anger as predictors of CHD. Despite the impressive accumulation of evidence with respect to these psychosocial predictors of CHD, inconsistencies also emerged, as is usual with research on the relationship between personality and health (Holroyd \& Coyne, 1987).

The aim of the present chapter is to provide an overview of the findings regarding the association between hostility and health. In the first section an attempt is made to place the current interest in the role of hostility and anger in the development of CHD in a historical context.

Next, several important conceptual issues are discussed. This section includes definitions of the related concepts of anger, hostility, and aggression.

The third section deals with the procedures available for assessing hostility and anger. Various instruments that have been employed to measure hostility will be discussed; self-report "paper-and-pencil" tests as well as interview-based methods.

In the fourth section, a review of the evidence of a relationship between hostility and anger, and CHD is presented. A distinction is made between findings of prospective and those of cross-sectional studies.

The fifth section addresses the question of potential mechanisms linking hostility to CHD. An inventory is made of the proposed models of plausible underlying mechanisms.

Lastly, the literature on the developmental antecedents of hostility is summarized. Attention is paid to findings from socialization as well as behavioral genetics research. 


\subsubsection{From Type A to hostility: the story of coronary-prone behavior}

In the past few decades, the role of psychological and behavioral factors in the development of CHD has received much scientific and popular attention. The idea that psychological characteristics or behaviors may have a serious impact on the development of heart disease is not a new one, however. For instance, the wellknown 17 th century physician William Harvey described one of his patients with an acute bout of coronary ischemia as having "received an injury and affront from one more powerful than himself, and upon whom he could not have his revenge, was overcome with anger and indignation which he yet communicated to no-one..." (cited in Leibowitz, 1970). In $1673 \mathrm{~J}$. Archer used words of similar import: "The observations I have made in the practice of physicks these several years have confirmed me in this opinion, that the origin or cause of most men and women's sickness, disease and death, is first some great discontent which brings a habit of sadness of mind" (cited in Siegman, 1994a).

Until the beginning of the twentieth century knowledge of the potential influence of psychosocial factors on the etiology of heart disease was primarily anecdotal. Systematic investigation of psychological or behavioral risk factors of coronary disease started with the emergence of the field of psychosomatic medicine. This new field of interest originated from the ideas and clinical work of Sigmund Freud and his followers (e.g. Alexander, 1939; Arlow, 1945; Dunbar, 1943; Kemple, 1945; Menninger \& Menninger, 1936). On the basis of their clinical experience, these psychodynamically-oriented writers postulated that psychological conflicts influence pathological processes. This line of research consisted mainly of descriptions of constellations of behaviors and emotions, reported by groups of coronary patients during interviews, and interpretations of protocols of projective teclaniques like the Rorschach test. According to this psychoanalytic version of psychosomatic medicine, coronary patients were best characterized as having aggressive tendencies (often repressed), an achievement-oriented disposition, and the need to dominate. Unfortunately, the psychosomatic studies mentioned above all suffered from serious methodological flaws and have had little effect on research and the development of theories concerning the risk for CHD.

Direct systematic observation of personality and/or behavioral risk factors of CHD is to be found in the work of the two cardiologists. Friedman and Rosenman. Their search for "coronary-prone behavior" was primarily started off by the fact that the traditional risk factors of CHD, such as smoking, hypertension, family history of CHD, hyperlipidemia, and obesity, could explain only about $50 \%$ of CHD cases (Jenkins, Zyzanski, \& Rosenman, 1979; Keys, 1970; Rosenman, 1983). In the late $1950 \mathrm{~s}$, they identified a common configuration of behaviors that they considered to be risk factors of heart disease in many of the coronary patients in their practice. This cluster of behaviors consisted of impatience, time urgency, extreme ambition, and aggressive and hostile behavior and was labeled the "Type A coronary-prone behavior pattern" (TABP). Friedman and Rosenman defined this behavior pattern as 
"an action-emotion complex that can be observed in any person who is aggressively involved in a chronic, incessant struggle to achieve more and more in less and less time and, if required to do so, against the opposing efforts of other things or other persons" (Friedman \& Rosenman, 1974, Rosemman \& Friedman, 1974). "The opposite of Type A, labeled "Type B behavior" was initially defined as the relative absence of most of the Type A behaviors. Now and then, however, it was suggested that Type B behavior is not just the absence of Type A characteristics but, in fact, a different coping style in its own right (e.g. Rosenman \& Chesney, 1982; Siegman \& Dembroski, 1989).

The assessment method that was developed by Rosenman and Friedman to measure the TABP included a structured interview (SI) and rating of Type A features, focusing particularly on overt manifestations of this behavior pattern. These manifestations are explosive, accelerated speech, impatience, tense facial musculature, and an inclination to challenge and compete with other persons even in non-competitive situations (Rosenman, 1978).

In order to examine the predictive validity of the TABP for CHD, the Western Collaborative Group Study (WCGS) was launched. This large-scale prospective study provided the first evidence that the TABP was associated with an increased risk for clinically manifest CHD. This risk was found to be independent of the classic risk factors of CHD, hypertension, smoking, and high cholesterol (Rosenman et al., 1975). A similar finding was obtained in the Framingham Study, a longitudinal study of cardiovascular disease with a short self-report measure for the TABP (Haynes, Feinleib, \& Kannel, 1980). Further evidence of an association between the TABP and heart disease outcomes has been provided by numerous other studies (e.g. Blumenthal, Williams, Kong, Schanberg, \& Thompson, 1978; Frank, Heller, Kornfeld, Sporn, \& Weiss, 1978; Friedman, Rosenman, Straus, Wurm, \& Kositchek, 1968).

It was mainly on the basis of the convincing epidemiologic evidence generated by the prospective investigations of the WCGS and the Framingham Study that a panel of distinguished researchers, who met in 1978, concluded that the TABP should be considered an independent risk factor of CHD (Cooper, Detre, \& Weiss, 1981). Shortly after this conference, the tide started to turn as a number of studies, both prospective and retrospective, reported a lack of association between the TABP and coronary disease outcomes. For instance, prospective investigations in Great Britain and the Netherlands failed to find significant associations between the TABP and the incidence of major CHD events (Appels et al., 1987; Johnstor, Cook, \& Shaper, 1987). But even more important in this regard were the negative results of the Multiple Risk Factor Intervention Trial (MRFIT), a prospective study of individuals at high risk for CHD (Shekelle et al., 1985). In this study, the TABP falled to predict CHD and was found to be unrelated to recurrent coronary events among subjects with previous myocardial infarction (Case, Heller, Case, \& Moss, 1985; Shekelle, Gale, \& Norusis, 1985). Besides, analysis of 22-year follow-up data of the WCGS showed that the TABP was not predictive of CHD incidence. Neither was the TABP predictive of recurrent $C H D$ among patients who survived a myocardial infarction 
during the initial 8/2-year period of the study (Ragland \& Brand, 1988a, 1988b). In addition, a number of other studies failed to replicate earlier results regarding the association between the TABP and CHD (e.g. Arrowood, Uhrich, Gomillion, Popio, \& Raft, 1982; Dimsdale, Hackett, \& Hutter, 1979; Krantz, Sanmarco, Selvester, \& Mathews, 1979).

These findings have called into question the risk factor status of the TABP with regard to coronary disease. However, even before the first negative findings were published, investigators had begun to look for components of the global TABP that might have special coronary-prone relevance. Reanalysis of the $81 / 2$-year follow-up WCGS data demonstrated that several components of the global TABP, such as "anger directed outward", "experience of anger more than once a week", "vigorous answers", "competitiveness", "explosive voice", "irritability", and, in particular, "potential for hostility" discriminated between future cases and non-cases (Matthews, Glass, Rosenman, \& Bortner, 1977). Using the same data base, Jenkins, Zyzanski, and Rosenman (1978) proved that different sets of components of the global TABP were the best predictors of different manifestations of $\mathrm{CHD}$, like angina pectoris, silent MI, and symptomatic MI.

The search for the really "toxic" elements of the overall TABP was further stimulated by the findings of a study of a group of patients referred for diagnostic coronary angiography. In these patients, both SI-defined TABP and self-reports of hostility, as measured by the Cook-Medley Hostility subscale from the MMPI, were significantly associated with severity of coronary artery disease (CAD). Of the two factors, however , hostility was found to be most strongly related to severity of CAD (Williams et al., 1980). Subsequent research on the important elements of the broadly defined TABP has focused predominantly on hostility and anger and related concepts (Dembroski \& Costa, 1987).

Before reviewing the results concerning hostility and health that have been collected in recent years, we will first discuss some important issues with respect to the definition of central concepts and measurement.

\subsubsection{Conceptual definitions}

It has frequently been pointed out in the literature that the study of hostility as a potential risk factor of disease suffers from a lack of clearly defined central concepts. Tescing theories and models of psychosocial effects on health is of great importance to health psychology. However, an unambiguous and comprehensive framework of constructs is fundamental to such tests, because the inadequate definition of concepts threatens the validity of research outcomes (Campbell \& Stanley, 1963).

The concepts of anger, hostillity, and aggression, although closely related, are not identical. Nevertheless, the three constructs are frequently differentiated insufficiently from each other or even used interchangeably. Previous discussions of the differences between emotion, cognition, and behavior are useful in this respect (Buss, 1961; Izard, 1979; Spielberger et al., 1985; Zillmann, 1979). 
Anger is defined as an emotional state composed of feelings ranging in intensity from mild irritation or annoyance to fury and rage. This emotional state is usually evoked when a person is blocked in the attaimment of a goal or in the fullilment of need or when a subject feels mistreated (Diamond, 1982). Anger can be conceived as an emotional state and as a permanent personality trait. Anger as a personality trait implies individual differences in the frequency and intensity of this emotion (Spielberw ger et al. 1985). A related concept is anger expression, which refers to the way in which feelings of anger are communicated, in particular whether angry feelings are kept in (anger-in) or acted out (anger-out).

Aggression, though frequently related to anger, refers to overt behavior. It generally consists in destructive or punitive behavior directed at other people or objects (Spielberger, Jacobs, Russell, \& Crane, 1983). In the past, several investigators (Averill, 1982; Bandura, 1973; Megargee, 1985) have distinguished a number of distinct types of aggressive behavior, varying from direct (e.g. assault), indirect (e.g. making aggressive noises), and passive physical aggression (e.g. failure to keep a promise) to verbal aggression (e.g. sarcasm, rudeness, argumentativeness, and insults).

Finally, as for hostility, several researchers have given different definitions of this concept, thereby clouding the distinction between emotion, cognition, and behavior. One well-known and influential approach considers hostility to be a complex set of negative attitudes, beliefs, and appraisals which arises from an absence of trust in the fundamental goodness of other people and is focused on the belief that other people are generally mean, selfish, and not to be trusted (Williams, Barefoot, \& Shekelle, 1985). In a recent elaboration of this matter, Barefoot (1992) postulated that hostility should be divided into affective, behavioral, and cognitive components, and that these components doubtless covary, but do not necessarily always occur together. Therefore, Barefoot argues, these elements of hostility should be defined and measured separately. According to this approach, the affective component of hostility consists of several emotional states, such as anger, annoyance, resentment, disgust, and contempt. The cognitive component of hostility includes negative beliefs about other people (i.e. cynicism). Hostile persons typically consider their fellowmen to be immoral, untrustworthy, and dishonest. Consequently, hostile individuals are prone to perceive the behavior of others as threatening or antagonistic (i.e. hostile attributions), so that, from the hostile person's point of view, other people's behavior justifies his/her own antagonistic behavior. Finally, the behavioral component of hostility comprises aggression and a range of more or less subtle forms of antagonistic behavior.

In conclusion, this survey of conceptual definitions clearly shows that it is not easy to disentangle the closely related concepts of anger, hostility, and aggression, which show considerable overlap. This overlap should not come as a surprise, as it is known from the literature that personality traits can be defined in terms of affective, cognitive, and behavioral characteristics. However, it has also been argued that individual aspects of global traits may be differentially associated with health (Car- 
ver, 1989). Therefore, clear definitions of key concepts are prerequisite for real and substantial progress in the field of behavioral medicine.

\subsubsection{The assessment of hostility}

In addition to the issue of conceptual definitions dealt with in the previous section, measurement issues are also essential to the study of the relationship between hostility and health. As was indicated before, hostility, anger, and aggression are considered to be separate entities. However, at the same time it is obvious that these constructs are interrelated. There are many different assessment devices for measuring hostility (Matthews, Jamison, \& Cottington, 1985). These measures of hostility appear to be only moderately intercorrelated (Musante, MacDougall, Dembroski, \& Costa, 1989). The moderate associations may be explained by the fact that different measures reflect different elements of hostility (affects, cognitions, and behavior). If it is assumed that all instruments assess the same broad concept of hostility, this may easily lead to inconsistent research findings, thus lowering the status of the hypothesis that there is a relationship between hostility and health.

Research on hostility as a psychosocial risk factor for CHD can be classified into two general approaches: 1) hostility or anger as assessed by the SI or by later modified versions of this method, with the rating focusing on overt behavioral expressions indicative of hostile tendencies or anger; and 2) hostility or anger as measured by self-report questionnaires. More recently, peer reports have been proposed as an additional method for the measurement of hostility and anger (Barefoot \& Lipkus, 1994; Buss \& Perry, 1992; Kneip et al., 1993). However, because peer reports have, so far, seldom been employed in hostility studies, the present discussion will focus on self-report and interview-based hostility assessments. The following sections do not purport to be a comprehensive inventory of the available instruments; only measures that have been used in CHD research will be discussed.

\subsubsection{Interview-based assessments of hostility and anger}

Since the end of the 1970 several systems bave been developed on the basis of clinical judgments of behavior. These procedures all rate behavior during the SI, which was originally designed for the measurement of the TABP in the WCGS (Rosenman, 1978). Although they seem quite similar at first sight, they differ to such an extent that separate discussion seems appropriate.

The earliest procedure was originally dleveloped by Bortner and used in the study by Mathews et al. (1977). This component scoring system consisted of separate ratings of 40 features, including several hostility-related characteristics, in structured interview responses. In spite of positive results, the Bortner system has not been employed in subsequent studies on psychosocial risk factors of CHD, which is probably due to the untimely death of the author. 


\section{Hostility Facet Scoring System (HFSS)}

Component scoring of the SI was further refined by Dembroski and his co-workers (Dembroski \& Costa, 1987). For the sake of convenience this scoring system is labeled the HFSS (Barefoot, 1992). The HFSS makes a distinction between Toral Potential for Hostility (PH) and three hostility components: Content, Intensity, and Style. Potential for hostility is conceptually defined as "the relatively stable tendency (a) to experience varying degrees and combinations of anger, irritability, resentment, and related negative affects in response to common, everyday events that are likely to arouse them in individuals who are prone to react in such ways, and/or (b) to react with expressions of antagonism, disagreeableness, rudeness, surliness, criticalness, and uncooperativeness" (Dembroski \& Costa, 1987, p. 224). In the initial versions of the HFSS, only PH was rated on a 5-point scale after listening to an entire interview (Dembroski \& MacDougall, 1983; MacDougall, Dembroski, Dimsdale, \& Hackett, 1985). However, recently the system has been extended to include separate assessments of content, intensity, and style, which are also based on the entire interview (Dembroski, MacDougall, Costa, \& Grandits, 1989). Hostile content refers to the degree to which the interviewee reports anger and hostility. The rating of Intensity is based on the intensity of the expression of hostile and negative feelings. Hostile style denotes uncooperative, disagreeable, and rude behavior exhibited during the interview.

Interrater reliability of ratings of $\mathrm{PH}$ and its three components have been found to be satisfactory, ranging from 0.70 to 0.85 (e.g. Dembroski, MacDougall, Williams, Haney, \& Blumenthal, 1985; Dembroski et al., 1989; Musante et al., 1989). As yet, there is very little evidence with respect to the construct validity of HFSS ratings. Significant (but modest) relationships between PH ratings and scores on the Cook and Medley (1954) Hostility (Ho) Scale have been reported, with correllations ranging between 0.29 and 0.37 (Dembroski et al., 1985; Swan, Carmelli, \& Rosenman, 1990). In a study of male and female children and adolescents PH proved to be significantly associated with anger expression (self-report) in boys, but not with scores on an adapted version of the Ho scale for either group (Woodall \& Matthews, 1989). Furthermore, results of a study of college students revealed that PH was related to self-reported antagonistic interactions with other people but not to peer reports of agreeableness versus antagonism (Costa, McCrae, \& Dembroski, 1989). Generally, PH ratings seem to be significantly related to the expressive facets of hostility (manifest behaviors such as assaultiveness, verbal aggression, and rudeness). Experiential hostility (cognitive and emotional processes like resentment and suspicion), on the other hand, appears not to be associated with ratings of PH.

In sum, these results lend some support to the construct validity of SI-defined hostility. However, further examination of validity issues with respect to the HFSS will be necessary. 


\section{Component Scoring System (CSS)}

The CSS is a rating method of taped SI responses, related to the HFSS (Chesney, Hecker, \& Black, 1988; Hecker, Chesney, Black, \& Frautschi, 1988). The CSS is a more elaborate scoring system than the HFSS. It was constructed to code 14 dimensions of the TABP, including hostility. The operational definition of hostility is very similar to the construct of Potential for Hostility of the HFSS. One important difference is that in the CSS the recorded structured interviews are divided into 20 segments, whereas researchers employing the HFSS base their rating of PH on a global impression of the entire interview. Each unit of the interview is rated with respect to hostility, using a 4-point scale. This innovation facilitates the achievement of good interrater reliability. However, to date scarcely any evidence has been produced. to support the construct validity of this assessment procedure.

\section{Interpersonal Hostility Assessment Technique (IHAT)}

The IHAT is the most recently developed rating system for hostility (Barefoot, 1992; Barefoot \& Lipkus, 1994). It is based on both the HFSS and the CSS and incorporates the best elements of these two earlier procedures. The way of scoring is very similar to that of the CSS, while, in accordance with the HFSS, a distinction is made between Hostile Content and Stylistic Hostility. Each interview question is rated on four specific hostility categories; Hostile Evasions, Indirect Challenges, and Direct Challenges refer to the behavioral aspects of hostility, whereas Irritation refers to the affective component. These four categories of hostile behavior may also be summed to yield a broad Hostile Behavior index. It is characteristic of the IHAT that more emphasis is placed on the respondent's style of interaction with the interviewer than on the content of replies. Thus far, high levels $(r=0.90)$ of interrater reliability have been reported (Barefoot \& Lipkus, 1994), but, as is the case with the CSS, studies on the construct validity still need to be done.

\subsubsection{Self-report measures of hostility and anger}

In addition to a large number of interview-based assessment systems, there are also numerous self-report instruments to measure hostillity and anger (Matthews et al., 1985). The review presented here only concerns measures that have been used in empirical studies of hostility and CHD.

\section{Cook-Medley Ho Scale}

The Ho scale, which has been derived empirically from the MMPI, is the measure of hostility used most frequently in research in the field of behavioral medicine. It was originally constructed to discriminate between teachers with good and those with bad relations with their students (Cook \& Medley, 1954). The widespread ase of the Ho 
scale is attributable to the fact that in both cross-sectional and prospective studies Ho scores have been found to be associated with various health outcomes, such as myocardial infarction, coronary artery disease, and total premature mortality. Negative findings have also been reported, but the bulk of the evidence suggests that the Ho scale measures something that is connected with health.

Though the Ho scale is considered to be an index of general hostility in most epidemiological studies, its construct validity has been questioned more than once (e.g. Matthews, 1985; Megargee, 1985; Rosenman, Swan, \& Carmelli, 1988; Smith, 1992). Comparatively high levels of internall consistency have been reported in the literature, with average Cronbach's $\alpha$ "s of roughly 0.80 (Smith \& Frohm, 1985). Notwithstanding this high internal consistency, several studies which applied factor analysis to the Ho scale yielded different outcomes, indicating that its internal structure seems not to be invariant across studies. Two studies observed one stable factor labeled cynical mistrust (Greenglass \& Julkunen, 1989; Smith \& Frohm, 1985), whereas another study suggested the presence of two factors, defined as cynicism and paranoid alienation (Costa, Zonderman, McCrae, \& Williams, 1986). In addition, Barefoot, Dodge, Peterson, Dahlstrom, and Williams (1989) introduced a conceptual content analysis. On the basis of the face validity of the individual items, they distinguished several clusters of items. Three subsets of items apparently referred to the affective, cognitive, and behavioral dimensions of hostility (Cynicism. Hostile Affect, and Aggressive Responding). A fourth cluster appeared to reflect the inclination to interpret other people's behavior as hostile and threatening (Hostile Attribution). A fifth subset of items indicated a subject's tendency to avoid other people or to shum social interactions (Social Avoidance). Finally, several items were considered to assess something else than hostility (Other). Very recently, Lipkus, Barefoot, Beckham, and Haney (1993) identified three dimensions by means of multidimensional scaling procedures, viz. social withdrawal/paranoia versus proactive aggression, rigidity of morai standards, and aggression versus cynicism.

The heterogeneity of Ho scale items may be attributed to the origins of the scale, which was initially an empirically derived MMPI scale to assess teachers' aptitude for classroom teaching. At the same time, however, this item heterogeneity may contribute to the inconsistency of findings regarding the association between hostility and health.

Evidence concerning the stability of Ho scores is mixed. Two studies, which used samples of medical students and middle-aged adult subjects, observed 1-year and 4year test-retest reliability coefficients greater than 0.80 (Barefoot, Dahlstrom, \& Williams, 1983; Shekelle, Gale, Ostfeld, \& Paul, 1983). However, the results of a study of college students - with a 22-year gap in testing - found Ho scores to be considerably less stable over time, the test-retest correlation coefficient being 0.39 (Siegler et al., 1990). More recently, Woodall and Matthews (1993) reported a 4year test-retest correlation of 0.56 , using an adjusted version of the Ho scale in a study of 10-18-year-old children and adolescents. It is interesting to note that Ho scores proved to be more stable for girls $(0.65)$ than for boys $(0.29)$. 
Several studies have revealed significant relationships between the Ho scale, other self-report measures of anger and hostility (Barefoot et al., 1989; Smith \& Frohm, 1985; Smith, Pope, Sanders, Allred, \& O'Keeffe, 1988; Woodall \& Mathews, 1989), and indices of hostility in role playing of conflict (Hardy \& Smith, 1988), which indicates that the scale has convergent validity. Additionally, the study by Smith and Frohm (1985) suggests that the Ho scale is more strongly correlated with experiential aspects of hostility (e.g. resentment or suspicion) than with more overt forms of hostility, such as assaultiveness or verbal aggression. Evidence with respect to the discriminant validity of the Ho scale varies. Smith and Frohm (1985) demonstrated that Ho scores correlated more strongly with self-report measures of anger proneness than with measures of anxiety and depression. In line with this finding, Barefoot et al. (1989; Study 1) found the Ho scale to be more closely correlated with the dimension of antagonism versus agreeableness than with the dimensions of neuroticism, openness to experience, extraversion, or conscientiousness of the NEO Personality Inventory (Costa \& McCrae, 1985a). However, results from another sample of the same study (Barefoot et al., 1989; Studly 2) showed that Ho scores were strongly related to neuroticism and general emotional distress. As several studies have suggested that measures of neuroticism are not associated with disease (Costa \& McCrae, 1985b, 1987; Schroeder \& Costa, 1984; Watson \& Pennebaker, 1989), the correlation between the Ho scale and this global trait may result in diminished predictive power of the scale.

As yet, very little evidence is avallable regarding the overt behavioral correlates of the Ho scale. As was remarked before, Ho scores have been shown to be significantly related to behavioral ratings of Potential for Hostility (Dembroski et al., 1985; Swan et al., 1990). High scores on the Ho scale proved to be associated with significant increases in self-reported anger and overt hostile behavior during discussions of conflictual topics in the marital context, although this association applies to males in particular (Smith, Sanders, \& Alexander, 1990).

Several investigations have provided support for the cognitive underpinning of Hodefined hostility. It was shown that Ho scores were significantly more closely related to daily accounts of suspicious and angry thoughts than to depressed or anxious thoughts (Pope, Smith, \& Rhodewalt, 1990). Furthermore, individuals with high Ho scores were found to be more inclined to interpret other people"s and their spouse's behavior as intentionally provoking conflicts. The latter, however, only applies to husbands (Pope et al., 1990; Smith et al., 1990). Finally, a study using an information-processing model, found that highly hostile subjects had increased recall of hostile trait adjectives (e.g. unfriendly, rude) following hostile social interaction (Allred \& Smith, 1991).

The present discussion of reliability and validity issues illustrates the psychometric problems concerning the Ho scale. Yet, there is general consensus that the bulk of the Ho items primarily reftect the cognitive aspects of hostility, i.e. cynical beliefs and basic mistrust of other people. 


\section{Buss-Durkee Hostility Inventory (BDHI)}

The BDHI (Buss \& Durkee, 1957) is a comprehensive self-report questionnaire, designed to assess both an individual's level of general hostility and separate aspects of hostility. The scale consists of seven subscales: Assault, Indirect Hostility, Irritability, Negativism, Resentment, Suspicion, and Verbal Hostility. The subscale Suspicion predominantly measures the cognitive dimension of hostility, while aspects of the affective component are reflected by the Irritability and Resentment subscales. The behavioral aspects of hostility are represented by the subscales Assault, Indirect Hostility, Negativism, and Verbal Hostility, which constitute different styles of expressing hostility. The original study by Buss and Durkee (1957) factor analyzed scales, which yielded two factors. Factor 1 was called Aggressiveness (consisting of the subscales Assault, Indirect Hostility, Irritability, and Verbal Hostility). The second factor was called Hostility (composed of the subscales Resentment and Suspicion). Subsequent factor-analytic studies have produced different findings. Bendig (1962) found two factors; one, consisting mainly of Irritability items, was labeled Covert Hostility, whereas the other, composed of items of the Assault and Verbal Hostility subscales in particular, was labeled Overt Hostility. However, although results of various factor-analytic studies (e.g. Bendig, 1962; Bushman, Cooper, \& Lemke, 1991; Buss \& Durkee, 1957; Musante et al., 1989) only show partial correspondence, they all provide substantial support for a distinction between experiential and expressive hostility. Experiential hostility is primarily represented by the Resentment and Suspicion subscales, and expressive hostility is mainly defined by the subscales Assault and Verbal Hostility. Further evidence of the presence of these two hostility dimensions as measured by the BDHI was provided by a recent study on the psychometric characteristics of a Dutch version of the BDHI. Two factors, called Overt and Covert Aggression, which appeared to be very similar to the factors experiential and expressive hostility, were distinguished (Lange et al., 1995).

Evidence with regard to the internal consistency and the temporal consistency of the BDHI is limited. Bendig (1962) reported Kuder-Richardson-20 coefficients for the Overt and Covert Hostility scales of 0.76 and 0.72 , respectively. Lange et al. (1995) found Cronbach's $\alpha$ 's of $0.79,0.77$, and 0.80 for Covert Aggression, Overt Aggression, and total BDHI, respectively. Two-week test-retest reliability coefficients have been reported to vary from 0.64 to 0.78 for the BDHI subscales, and appeared to be 0.82 for the BDHI total score (Biaggio, Supplee, \& Curtis, 1981).

The work of Costa and his colleagues (1989) lends support to the construct validity of the distinction between experiential and expressive hostility. In their study, the association between experiential and expressive hostility on the one hand, and selfreports and peer ratings of the personality traits of neuroticism and agreeableness versus antagonism on the other were investigated. Neuroticism is a broad personality trait which refers to the inclination to experience negative emotions, such as depression, anxiety, and anger. Agreeableness stands for a polite, sympathetic, trusting, and cooperative style of interacting with other people. Antagonism, the other end of this 
dimension, is characterized by a cynical, mistrusting, insensitive, and uncooperative interpersonal style. Experiential hostility was found to be related to both neuroticism and antagonism, the association with neuroticism being more robust. Expressive hostility, on the other hand, proved to be correlated with antagonism, but no relationship was observed with neuroticism. So far, the scanty evidence available on the construct validity of the $\mathrm{BDHI}$ corroborates the distinction between the experiential hostility factor and the expressive hostility factor.

\subsubsection{Conclusion}

The above discussion of issues connected with the measurement of anger, hostility, and aggression clearly shows that a lot of work still needs to be done. The phenomena under scrutiny are complex, multidimensional, and conceptualized in various ways (Smith, 1994). Although there is good reason to assume that ratings of Potential for Hostility, the Ho scale, and the BDHI tap the concept of hostility, it is at the same time obvious that these measures should not be considered to be interchangeable; they assess related but different aspects of the multifaceted concept of hostility. These distinct components may not be equally toxic in the etiology of CHD or may work via different underlying mechanisms. This may hamper comparison of results across studies.

Compared to some ten years ago, considerable progress has been made in the field of hostility assessment. However, the findings of the hostility studies thus far, confront the field of behavioral medicine with a serious conflict between known health relevance and psychometric shortcomings which is very difficult to solve. This applies especially to the Ho scale. Its value as a measurement device for hostility may be reduced due to its strong correlation with neuroticism and the heterogeneity of item content, but the Ho scale may still be preferable to other, psychometrically more solid measures for research purposes on account of previous demonstrations of its relationship with adverse health outcomes in prospective investigations. In order to find a satisfactory solution to this major problem, additional prospective research is essential.

\subsubsection{Hostility and health: evidence of an association}

The association between individual differences in hostility and the coronary disease endpoints of myocardial infarction (MI), sudden cardiac death, and angiographically documented evidence of coronary artery disease (CAD) has been investigated extensively. This research can be divided into cross-sectional and prospective studies. Because these two kinds of studies are quite different in nature, they will here be discussed in separate sections. Furthermore, the studies discussed here are grouped into studies using self-report measures of hostility and SI-based assessments of hostility. The majority of studies concerning the hostility-CHD hypothesis employ either 
the Ho scale or the SI derived hostility subcomponent. In cases where other measure ment devices are used this is indicated.

\subsubsection{Cross-sectional studies}

For the sake of clarity, it should be noted that the following survey of cross-sectional studies will present the results yielded by two types of cross-sectional studies. Firstly, case-control studies comparing CHD patients with a control group of persons without CHD will be discussed. Secondly, coronary angiography studies, examining subjects at high risk for CHD by means of cardiac catheterization, will be reviewed. A concise summary of case-control studies is presented in table 1.1.

\section{Case-control studies}

In total, 14 cross-sectional studies evaluating the association between hostility and CHD have been published. Six of them will only be mentioned briefly here, because of their substantial methodological drawbacks, which are e.g. the use of only one item to assess hostility (Theorell, 1973; Wardwell, Bahnson, \& Caron, 1963), raters not being blind to group status (Miles, Waldfoger, Barrabee, \& Cobb, 1954; Miller, 1965), the inclusion of an unsuitable reference group, such as spouses of coronary patients (Croog, Koslowski, \& Levine, 1976; Theorell, DeFaire, Schalling, Adamson, \& Askevold, 1979), and significant loss of subjects from the original study sample (Miles et al., 1954; Theorell et al., 1979). Eight cross-sectional studies appear to be methodologically sound and will be dealt with in more detail.

In a Swedish study (Bengtsson, Hallstrom, \& Tibblin, 1973), 42 post MI (6-36 months) females and a subsample of 68 women free from coronary disease were compared. Results indicated that scores on the Aggression subscale of the CesarecMarke Personality Schedule significantly discriminated cases from controls.

Findings of a study by van Dijl (1982), who compared male MI cases with a control group matched for age, sex, and eclucational level, all from the Netherlands, in three independent samples consisting of 204, 196, and 126 subjects respectively, showed that MI cases exhibited higher levels of hostility than control subjects, as measured by a nime-item Dutch aggressiveness/hostility scale.

In a random sample of 4,462 young male Vietnam veterans (age range 31 to 46 years), Joesoef, Wetterhall, DeStefano, Stroup, and Fronek (1989), demonstrated a significant association between Ho scores and the prevalence of peripheral artery disease, after adjustment for standard risk factors in multivariate analyses.

Two other case-control studies have also used the Ho scale. One study (Friedman \& Booth-Kewley, 1987) reported no differences in average scores on the Ho scale between $50 \mathrm{CHD}$ patients and 50 control subjects. Fontana et al. (1989) did not find any significant differences in mean Ho scores between a group of 23 CHD patients and a control group consisting of 41 medical and surgical patients either. 
Table 1.1. Cross-sectional studies of hostility and CAD

\begin{tabular}{|c|c|c|c|}
\hline Study & Subjects & Hostillity measure & Result \\
\hline $\begin{array}{l}\text { Barefoot et al., } \\
1994\end{array}$ & $\begin{array}{l}24 \text { males with some evidence } \\
\text { of CAD, } 25 \text { male controls free } \\
\text { from CAD. Samplle from } \\
\text { Air Force population. }\end{array}$ & $\begin{array}{l}\text { Cook-Medley Ho scale. } \\
\text { SI hostility. }\end{array}$ & $\begin{array}{c}\text { ns } \\
+\end{array}$ \\
\hline $\begin{array}{l}\text { Bengtsson et al. } \\
1.973\end{array}$ & $\begin{array}{l}42 \text { female MI patients and } \\
68 \text { randomly selected control } \\
\text { females. }\end{array}$ & $\begin{array}{l}\text { Aggression trait from } \\
\text { Cesarec-Marke personality } \\
\text { schedulle. }\end{array}$ & + \\
\hline Croog et al., 1976 & $\begin{array}{l}283 \text { male MI patients, "non- } \\
\text { cardiac" wives as controls. }\end{array}$ & $\begin{array}{l}\text { Self-report on how } \\
\text { easily angered. }\end{array}$ & + \\
\hline van Dijl, 1982 & $\begin{array}{l}\text { Male MI patients and "healthy" } \\
\text { male controls, matched for age } \\
\text { and educational level. } \\
\text { Three independent samples: } \\
204,196 \text {, and } 126 \text { subjects. }\end{array}$ & $\begin{array}{l}\text { Dutch Aggressiveness/ } \\
\text { Hostility scale. }\end{array}$ & + \\
\hline Fontana et al., 1989 & $\begin{array}{l}64 \text { male medicat and surgical } \\
\text { patients; } 23 \text { with CHD and } 41 \\
\text { without history of CHD. } \\
\text { Matched for SES and race. }\end{array}$ & $\begin{array}{l}\text { Cook-Medley Ho scale. } \\
\text { Full scalle score. } \\
\text { Dichotomized Ho } \\
\text { scores. }\end{array}$ & $\begin{array}{l}\text { ns } \\
+\end{array}$ \\
\hline $\begin{array}{l}\text { Friedman \& } \\
\text { Booth-Kewley, } \\
1987\end{array}$ & $\begin{array}{l}50 \text { male MI patients and } 50 \\
\text { healthy controls. Matched for age, } \\
\text { ethnicity, income, and education. }\end{array}$ & Cook-Medley Ho scale. & ns \\
\hline Joesoef et al, 1989 & 4,462 young male veterans. & Cook-Medley Ho scale. & + \\
\hline $\begin{array}{l}\text { Koskenvuo et al. } \\
1988\end{array}$ & 3.750 Finnish male twins. & $\begin{array}{l}\text { 3-item hostility } \\
\text { questionnaire. }\end{array}$ & + \\
\hline $\begin{array}{l}\text { Mendes de Leon, } \\
1992\end{array}$ & $\begin{array}{l}31 \text { male MI patients, } 26 \text { male } \\
\text { patients with unstable angina } \\
\text { and } 26 \text { male hospital controls. }\end{array}$ & $\begin{array}{l}\text { State-Trait Anger Scale } \\
\text { trait wersion. } \\
\text { Anger Expression Scale. } \\
\text { Impatience/Irritability } \\
\text { scale from the Jenkins } \\
\text { Actiwity Survey (JAS). }\end{array}$ & $\begin{array}{l}\mathrm{ns} \\
+ \\
+\end{array}$ \\
\hline Miles et. al., 1954 & $\begin{array}{l}46 \text { young male } M I \text { patients } \\
\text { and } 49 \text { controls, who were } \\
\text { different in SES and education. }\end{array}$ & $\begin{array}{l}\text { Aggression derived from } \\
\text { psychiatric interview. }\end{array}$ & + \\
\hline
\end{tabular}


Table 1.I. (Contimued)

\begin{tabular}{llll} 
Study & Subjects & Hostility measure & Result \\
\hline Miller, 1965 & $\begin{array}{l}34 \text { MI patients and 34 controls. } \\
\text { Matched for age, sex, education, } \\
\text { and IQ. }\end{array}$ & $\begin{array}{l}\text { Hostility assessment } \\
\text { based on andlysis of } \\
\text { interview. }\end{array}$ & + \\
Theorell, 1973 & $\begin{array}{l}62 \text { male MI patients and 109 } \\
\text { randomly selected male controls. }\end{array}$ & One hostility item. \\
Theorell et al., 1979 & $\begin{array}{l}30 \text { male twin pairs. Divided into } \\
\text { a "healthier" and a "less } \\
\text { healthy" group. }\end{array}$ & $\begin{array}{l}\text { Buss Aggression } \\
\text { Inventory. }\end{array}$ & ns \\
Wardwell et al., 1963 & $\begin{array}{l}\text { 32 male MI patients and } 32 \text { age- } \\
\text { matched men hospitalized for } \\
\text { acute serious conditions. }\end{array}$ & One hostility item. \\
\hline
\end{tabular}

It is interesting, however, that when Ho scores were dichotomized, high hostility proved to be significantly associated with CHD.

In a sample of 3,750 Finnish male twins aged 40 to 59 , self-reported irritability, ease of anger arousal, and argumentativeness were related to heightened prevalence of angina pectoris (Koskenvuo et al., 1988).

Mendes de Leon (1992) compared 31 MI patients and 26 patients with unstable angina pectoris (AP) with 26 hospital control subjects, mainly orthopedic patients. It was observed that scores on the impatience/irritability scale of the Jenkins Activity Survey (JAS; Jenkins et al., 1979) and the Anger Expression Scale (Spielberger et al., 1985) were related positively to CHD, with a clear propensity toward outward expression of anger. Trait-Anger, as defined by the State-Trait Anger Scale (TSTAS; Spielberger et al., 1983), was found to be unrelated to CHD.

Recently, Barefoot et al. (1994) conducted an unusual case-control study of United States Air Force staff who had been referred for coronary angiography on account of the outcome of a standard noninvasive check-up. Hostility was measured by means of both behavioral ratings (SI) and self-report (Ho scale). This asymptomatic male sample consisted of 24 cases with some angiographic evidence of CAD and 25 controls without detectable evidence of CAD. Interview ratings of hostility were found to be significantly associated with the presence of occlusion, with cases displaying more hostile behaviors, whereas Ho scores failed to be significantly related with CAD. Further data-analysis demonstrated that interview-assessed hostility and history of smoking interacted significantly in such a way that hostility discriminated between diseased and healthy persons among nonsmokers, but not among smokers. 
These results support the theory that hostility has an effect on the early stages of CAD and suggest the presence of interactive effects of hostility and other risk factors.

As can be seen in table 1.1, eleven out of 14 case-control studies produced significant positive associations between hostility and coronary heart disease. As was noted before, the results of six studies must be interpreted with great caution, because of methodological weaknesses. However, eight studies appear to have been carried out properly, 7 of which suggest the existence of an association between hostility and cardiovascular disease.

\section{Coronary angiography studies}

Studies of patients undergoing coronary angiography can be divided into studies using self-report measures of hostility (especially the Ho scale) and studies employing behavioral ratings of hostility (in particular the SI). The studies in question are described briefly in table 1.2 .

\section{Self-report instruments}

In one of the first studies in this field, Williams et al. (1980) demonstrated a significant association between the Ho scale and coronary atherosclerosis in a sample of 424 patients (307 men and 117 women) who underwent diagnostic coronary arteriography for suspected CHD. Only $48 \%$ of patients with low Ho scores ( $\leq 10$ ) exhibited significant occlusion, whereas $70 \%$ of patients with high Ho scores $(>10)$ had significant coronary artery occlusion. Patients with Ho scores above 10 (ranging from 11 to a possible maximum of 50 ) were roughly at equal risk for occlusion, suggesting the presence of a threshold effect. The results of this study may have been affected by gender, as males and females were included in the same analysis. Because several studies indicated that women are less hostile than men (Barefoot et al., 1991; Blumenthal, Barefoot, Burg, \& Williams, 1987) and develop cardiovascular disease later in their life-span (Kannel \& Thom, 1994; Stoney \& Engebretson, 1994), the findings in Williams et al. may be explained by sex differences.

Two subsequent studies evaluating patients referred for coronary angiography failed to replicate the association between Ho scores and CAD. In a subset of 80 subjects from a sample of 131 patients (98 males and 33 females), Ho scores were found not to be significantly correlated with either the number of vessels with more than $75 \%$ occlusion or a total coronary index indicating CAD severity (Dembroski et al., 1985). Similarly, in a study of 158 hospitalized patients ( 118 men and 40 women) scheduled for cardiac catheterization. Helmer, Ragland, and Syme (1991) tested the association between the Ho scale and $\mathrm{CAD}$. Ho scores were analyzed in several ways: as a continuous measure, as a dichotomous measure, and using several cut-off points used in three previous studies (Barefoot et al., 1983; Shekelle et al., 1983; Williams et al., 1980). No significant positive associations were observed between 
any of the Ho scale measures and significant coronary occlusion $(\geq 75 \%)$ or mean occlusion in the four major coronary arteries. In addition, possible age and sex interactions of Ho scores with both significant occlusion and mean occlusion were examined. No significant interactions were found.

Table 1.2. Cross-sectional studies of hostility and CAD in coronary angiography patients

Study Subjects Hostility measture Result

Arrowood et al., Random sample of 76 patients. 1982

Dembroski et al., 98 men and 33 women referred 1985

Helmer et al., 1991

Helmers et al. 1993

Kneip et al., $1993 \quad 112$ men and 73 women referred

MacDougall et al, $\quad 126$ male patients referred 1985

Siegman et all., 1987

Stevens et âl., 1984

Williams et al.. 1980 for thallium stress testing. for coronary angiography.

51 men and 21 women referred for coronary angiography.

21 men and 23 women referred

for carotid artery angiography.

for coronary angiography.

Subsample of 80 patients.

118 men and 40 women referred for coronary angiograplyy.

63 male CAD patients and 17 female CAD patients referred for exercise tomographic thallium testing

307 men and 117 women referred for coronary angiography.
SI hostillity.

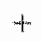

SI hostility.

Sl defined Anger-In.

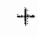

Cook-Medley Ho scale. ns

Cook-Medley Ho scale. ns

SI hostility. ns

Cook-Medley Ho scale. ns Cook-Medley Composite + Hostility score.

Multidimensional Anger

Inventory.

Self-rating.

Spouse-rating.

SI hostility.

SI defined Anger-In.

$+$

$+$

Buss-Durkee Hostility Inventory.

experiential hostility factor expressive hostility factor +

Jenkins Activity Survey + (JAS) hostility (3 items). curvi-

linear

Cook-Medley Ho scale. +

The relationship between different dimensions of hostility, as measured by the $\mathrm{BDHI}$, and CAD was investigated in a study of 72 subjects, 51 men and 21 women, 
undergoing coronary angiography (Siegman, Dembroski, \& Ringel, 1987). The number of vessels displaying more than $75 \%$ stenosis and an index of severity of atherosclerosis, consisting of both the extent of occlusion and the anatomic location of a lesion, were employed as the dependent variables. The results of this study revealed that experiential hostility - which the authors called "neurotic hostility" was inversely associated with CAD severity. Expressive hostility, on the other hand, showed a significant positive relationship with the extent of atherosclerosis. However, these associations were restricted to subjects younger than 60 ; among the older patients no significant relationships were observed.

Stevens, Turner, Rhodewalt, and Talbot (1984) carried out a somewhat umusual study in that they extended the study of the association between hostility and CHD to carotid artery atherosclerosis, which is associated with cerebral vascular disease rather than CHD. As atherosclerosis is supposed to be a generalized process occurring throughout the arterial system, it was assumed that the underlying biological mechanisms of coronary atherosclerosis and carotid atherosclerosis would be the same. Forty four consecutive patients ( 21 males and 23 females) were classified into three distinct groups. Classification was based on the estimation of obstruction. The first group showed no stenosis (less than $25 \% ; \mathrm{n}=17$ ), the second group had mild stenosis (between $25 \%$ and $75 \% ; \mathrm{n}=17$ ), and the third group displayed severe stenosis (more than $75 \% ; n=10$ ). Hostility, as defined by three items from the Jenkins Activity Survey (JAS; Jenkins et al., 1979), appeared to be marginally significantly associated with the severity of stenosis classes. Further exploration of the data revealed a curvilinear relationship: subjects in the severe stenosis group had the lowest hostility scores, patients with mild stenosis had the highest hostility scores, and subjects without stenosis exhibited intermediate hostility scores. Because gender was not included in the analyses, the results of this study may be biased if female subjects were affected by atherosclerosis to a lesser degree. Furthermore, a rather weak hostility measure (JAS) was employed.

Recently, Kneip et al. (1993) examined both self-ratings and spouse ratings of anger and hostility as predictors of CHD in 112 men and 73 women, who had been referred for thallium scintigraphy to assess chest pain or to check an abnormal electrocardiogram. Both cardiac patients and their spouses completed the Multidimensional Anger Inventory (MAI; Siegel, 1986) to measure multiple dimensions of anger and hostility. The MAI, originally a self-report questionnaire, was rewritten in the third person for spouses so that they could fill in the MAI with regard to their spouse. Self-rated MAI scores did not discriminate between patients with positive and patients with negative thallium scans. However, spouse ratings of Hostile Outlook and AngerIn were significantly associated with CHD, as evidenced by abnormal thallium scans. After adjusting for traditional CHD risk factors, this association still proved to be significant. The null findings with respect to patient-rated MAI subscales may be attributed to the significant inverse correlations of each MAI subscale with social desirability, which suggests that self-rated MAI scores are attenuated as social desirability increases. These findings further suggest the potential utility of spouse ratings 
of patient hostility, because of their presumed lower sensitivity to social desirability influences.

Another recent study investigated the relationship between hostility and the extent and severity of exercise-induced myocardial ischemia in 63 male patients and $17 \mathrm{fe}$ male patients with either documented $\mathrm{CAD}$ or a high probability of $\mathrm{CAD}$ (Helmers et al., 1993). To assess hostility, patients completed the Ho scale. Besides the full-scale score (Ho), a Composite Hostility (Chost) score (see Barefoot et al., 1989) was employed. Chost was found to be positively related to exercise-induced cardiac ischemia, whereas Ho was marginally significantly associated with exercise-induced cardiac ischemia. Repeated analyses for men and women separately, revealed that Ho failed to predict myocardial ischemia in the total sample of males $(n=63)$, the total sample of females $(n=17)$, and in middle-aged males (below 60 years of age) $(n=17)$. In contrast, Chost appeared to be significantly related to severity of exercise-induced ischemia in the complete study sample of 80 patients, in the subsample of women $(n=17)$, and in a subsample of middle-aged men $(n=17)$. The association between Chost and exercise thallium ischemia in the male sample $(n=63)$ failed to be significant, however. The findings of this study indicate that, as far as men are concerned, the relationship between hostility and exercise-induced ischemia may be limited to younger individuals. This means that the process of aging may have blurred this relationship in the total subsample of men. As it is well known that women develop CHD later in life than men do (e.g. Kannel \& Thom, 1994), it is quite possible that aging affected men and women differently in this study sample. The outcomes of this study underline the importance of Composite Hostility as a sensitive hostility measure. Furthermore, these results clearly indicate that it is better to study men and women as well as older and younger patient samples separately.

\section{Behavioral ratings}

Four studies of coronary angiography patients used behavioral ratings of hostility derived from the structured interview (SI). Using the early version of the Dembroski method for voice stylistics (Dembroski \& MacDougall, 1983), Arrowood at al. (1982) found the hostility component significantly related to degree of CAD in a random sample of 76 patients admitted for coronary angiography. The findings of this study should be treated with caution, as data on sociodemographic characteristics of the sample (such as age and sex) are lacking.

In two separate studies carried out by Dembroski, MacDougall, and co-workers, interview ratings of Potential for Hostility (PH) and Anger-In coping style were investigated in patients who had undergone diagnostic coronary catheterization (Dembroski et al., 1985; MacDougall et al., 1985). The first study included 131 coronary angiography patients (98 men and 33 women) from Duke University Medical Center (Dembroski et al., 1985). Patients had been selected from a larger sample and classified as having either no (0-vessel) CAD or severe (2- or 3-vessel) CAD. The disease endpoints in this study were the number of vessels displaying more than $75 \%$ occlu- 
sion, and a total coronary index, which was based on the sum of the extent of stenosis in all four cardiac vessels. Both $\mathrm{PH}$ and Anger-In proved to be significantly and positively associated with severity of CAD, i.e. patients characterized by high levels of PH and Anger-In had more diseased arteries and showed more unfavourable total coronary indices. Additional analyses demonstrated the presence of a significant interaction between PH and Anger-In for all disease endpoints, implying that high levels of $\mathrm{PH}$ are related to CAD only in those patients who had high Anger-In coping style scores, that is, who suppressed their anger. As was said before, Ho scale scores were avalable for 80 patients of this sample. However, in contrast with the positive findings regarding PH and Anger-In, Ho scores were not significantly correlated with either the number of obstructed arteries or the index of total CAD severity.

The data for the second study were obtained from 126 male patients who had been referred to Massachusetts General Hospital for diagnostic cardiac catheterization (MacDougall et al., 1985). The main purpose of this study was to replicate the findings of a previous study by Dembroski and colleagues employing a different population of coronary angiography patients. The results supported the previous findings: PH and Anger-In showed significant associations with the number of occluded coronary vessels. However, the significant interaction between PH and Anger-In found in the study by Dembroski et al. was not replicated.

The final study that is reviewed here has already been discussed in the section on self-report instruments (Helmer et a]., 1991). The Component Scoring System (CSS), developed by Hecker et al. (1988), was used to analyze interviews. The results revealed no significant positive association between SI-defined hostility and coronary occlusion. However, reanalysis of a subset of 124 structured interviews with the IHAT (Barefoot, 1992; Barefoot \& Lipkus, 1994) indicated that hostility ratings were strongly associated with severity of CAD (Barefoot, 1992; Barefoot et al., 1992). According to Barefoot and his colleagues, the hostility ratings reported in the article by Helmer et al. were relatively low, and it is quite possible that raters were inclined to register only the most striking examples of hostile behavior. As the more subtle demonstrations of hostility may be the strongest predictors of disease (Barefoot, 1993, personal communication), this may have led to the mull findings of Helmer and her co-workers. It should be noted, however, that this explanation is largely speculative and additional research will be necessary to be able to draw definite conciusions.

In sum, nine studies have been reviewed in this section. Eight studies included high-risk patients and one study used patients with either documented CAD and/or a high CAD probability. Seven studies produced positive relationships between hostility and several endpoints of CAD. Four studies made use of self-report assessments of hostility: Williams et al. (1980) demonstrated that hostility was significantly related to the presence of clinically significant atherosclerosis; Siegman et al. (1987) found a positive association between hostility and CAD in young patients, but not in older patients; Kneip et al. (1993) proved that spouse ratings of hostility were associated with thallium scans indicative of CHD; Helmers et al. (1993) showed that hostility was significantly correlated with CAD in female and middle-aged male patients, but 
not in older male patients. Four studies used a measure of hostility derived from the SI. Three studies revealed positive associations (Arrowood et al , 1982; Dembroski et al., 1985; MacDougall et al., 1985), whereas Helmer et al. (1991) failed to find a relationship. Two studies employed a self-report measure as well as behavional ratings of hostility: in the first study, only behavioral ratings of hostility were found to be related to CAD (Dembroski et al., 1985), whereas the second study failed to find any association at all (Helmer et al. 199l). Finally, Stevens et al. (1984) found a curvilinear association between hostility and carotid artery atherosclerosis.

\section{Cross-sectional studies: Pros and cons}

In this section on cross-sectional studies linking hostility and coronary heart disease, a total of twenty-three studies are reviewed. Generally, the findings of these studies lend some support to the existence of a relationship between hostility and CHD. Evidence supplied by studies using behavioral ratings of hostility appears to be more consistent than evidence yielded by studies employing self-ratings of hostility, in particular the Ho scale. However, one should be cautious about drawing conclusions, because of the relatively small number of studies using self-report measures of hostility other than the Ho scale.

Cross-sectional research has several attractive qualities. It can usually be done quickly, at relatively low cost, and frequently with large numbers of subjects. Crosssectional designs may also prove very useful for the investigation of possible associations between hostility and coronary disease to be tested in subsequent prospective studies, and for the study of diseases with low frequency. At the same time, however, these designs have several major limitations.

Due to the fact that the risk factor and the disease outcome are measured simultaneously in cross-sectional studies, these studies do not permit the testing of causal hypotheses. In this connection, Schlesselman already stated: "Thus, most observathonal studies end with an opinion or judgment about causality, not a claim of proof" (Schlesselman, 1982, p. 25). Furthermore, it is not clear whether behavior or a psychological state causes disease or whether it is the disease which causes behavior or a psychological state. In addition, cross-sectional designs also suffer from several other drawbacks that may lead to biased study results (e.g. Matthews, 1988). For instance, studies of patients referred for coronary angiography are vulnerable to patient selection bias. Selection bias may occur if only high-risk patients or patients with documented CAD are referred for cardiac catheterization. Thus, the association between hostility and cardiovascular disease may be weakened due to the restriction of range of variance in the severity of atherosclerosis. Besides, it is also quite possible that the range of hostility is restricted because patients at high risk for CAD will probably exhibit higher levels of hostility. As a result of this potential restriction of range of severity of CAD as well as hostility the strength of the association between hostility and disease may decrease (for reviews of this issue see Miller, Tumer. Tindale, Posavac, \& Dugoni, 1991; Pickering, 1985). 
"The study by Siegman et al. (1987) poins out another potential drawback of crosssectional studies using coronary angiography patients. Using the BDHI, Siegman and his colleagues found expression of hostility to be positively associated with severity of CAD. However, a significant negative correlation was observed between experience of hostility and CAD severity. Scores on a self-report measure of anxiety or neuroticism (Bendig, 1956) were also found to be inversely related to CAD severity in this study. Significant negative associations between neuroticism and CAD have been reported by several studies (Smith \& Williams, 1992). Costa et al. (1986) showed that neurotic indiwiduals are inclined to report chest pain in the absence of any evidence of coronary disease. If they continue complaining these persons will probably eventually be referred for diagnostic coronary angiography. This may result in the inclusion of patients exhibiting high levels of trait anxiety or neuroticism but free of $\mathrm{CAD}$ in catheterization samples. This means that the inverse association between neuroticism and $\mathrm{CAD}$ may be an artefact. Thus, dimensions of hostility that are highly correlated with neuroticism, such as the experiential hostility in the study by Siegman et al. (1987), may also show a spurious inverse association with CAD. Besides, hostility instruments measuring both expressive and experiential aspects of hostility may fail to find significant relationships with CAD in cardiac catheterization studies because of the opposite relationships of neuroticism and hostility with $\mathrm{CAD}$.

Retrospective bias or "the search for meaning" is another major threat to the validity of cross-sectional studies. This type of bias may occur when patients and their relatives are engaged in a search for the cause of the disease, which may result in an overrating of hostile characteristics.

In conclusion, cross-sectional studies of hostility and cardiovascular disease have yielded some evidence, but no consistent pattern of results. Therefore, it is too eariy to draw definite conclusions. Future cross-sectional research in this field will have to be especially wary of selection and measurement issues in order to avoid the abovementioned pitfalls.

\subsubsection{Prospective studies}

In the following section, studies of initially healthy individuals are distinguished from studies of coronary patients and high-risk patients. Furthermore, as in the previous section, studies using self-report measures of hostility and studies employing behavioral ratings of hostility are discussed separately. Table 1.3 gives a summary of the studies that are reviewed. 


\begin{tabular}{|c|c|c|c|}
\hline Study & Sulbjects & Hostility measure & Result \\
\hline Almada et al., 1991. & $\begin{array}{l}\text { Reanalysis of data from the } \\
\text { Western Electric Sudy (WES): } \\
1,871 \text { middle-aged men. } \\
25 \text {-year follow-up. }\end{array}$ & $\begin{array}{l}\text { Cynicism factor of the } \\
\text { MMPI. }\end{array}$ & + \\
\hline Barefoot et al., 1983 & $\begin{array}{l}255 \text { male medical students from } \\
\text { the University of North } \\
\text { Carolina. } \\
\text { 25-year follow-up. }\end{array}$ & $\begin{array}{l}\text { Cook-Medley Ho scale. } \\
\text { Low ws. high scores } \\
\text { (median). }\end{array}$ & + \\
\hline Barefoot et al., 1989 & $\begin{array}{l}118 \text { lawyers. } \\
28 \text {-year follow-up. }\end{array}$ & $\begin{array}{l}\text { Cook-Medley Ho scale. } \\
\text { Full Ho scale scores. } \\
\text { Composite Hostility score. }\end{array}$ & $\begin{array}{l}+ \\
+\end{array}$ \\
\hline Barefoot et al., 1987 & $\begin{array}{l}500 \text { older adults ( } 260 \text { men } \\
\text { and } 240 \text { women). } \\
15 \text {-year follow-up. }\end{array}$ & $\begin{array}{l}\text { Factor L of the } 16 \mathrm{PF} \\
\text { (suspiciousness). }\end{array}$ & + \\
\hline $\begin{array}{l}\text { Dembroski et al., } \\
1989\end{array}$ & $\begin{array}{l}\text { MRFIT study: } 192 \text { male high- } \\
\text { risk patients, who developed } \\
\text { CAD, and } 384 \text { matched high-risk } \\
\text { controls. } \\
\text { 7-year follow-up. }\end{array}$ & $\begin{array}{l}\text { SI hostility. } \\
\text { Low vs. high total } \\
\text { Potential for Hostility (PH). } \\
\text { Overall rating of PH. }\end{array}$ & ns \\
\hline $\begin{array}{l}\text { Hällström et al., } \\
1986\end{array}$ & $\begin{array}{l}795 \text { women. } \\
\text { 12-year follow-up. }\end{array}$ & $\begin{array}{l}\text { Aggression factor from } \\
\text { the Cesarec-Marke } \\
\text { Personality Schedule. }\end{array}$ & $\mathrm{ns}$ \\
\hline Haynes et al., 1980 & $\begin{array}{l}1,674 \text { men and women from the } \\
\text { Framingham Heart Study. } \\
\text { 8-year follow-up. }\end{array}$ & $\begin{array}{l}\text { Comprehensive psychosocial } \\
\text { questionnaite. } \\
\text { Males ( } 55 \text {-64 years old): } \\
\text { anger-out } \\
\text { anger-in } \\
\text { anger-discuss } \\
\text { Females ( } 45 \text {-64 years old): } \\
\text { anger-out } \\
\text { angerwin } \\
\text { anger-discuss }\end{array}$ & $\begin{array}{l}\mathrm{ns} \\
+ \\
\mathrm{ns} \\
\mathrm{ns} \\
+ \\
+\end{array}$ \\
\hline Hearn et al., 1989 & $\begin{array}{l}1,399 \text { male freshman students } \\
\text { of the University of Mimnesota. } \\
\text { 33-year follow-up. }\end{array}$ & Cook-Medley Ho scale. & $\mathrm{ns}$ \\
\hline
\end{tabular}


Chapter 1

Table 1.3. (Continued)

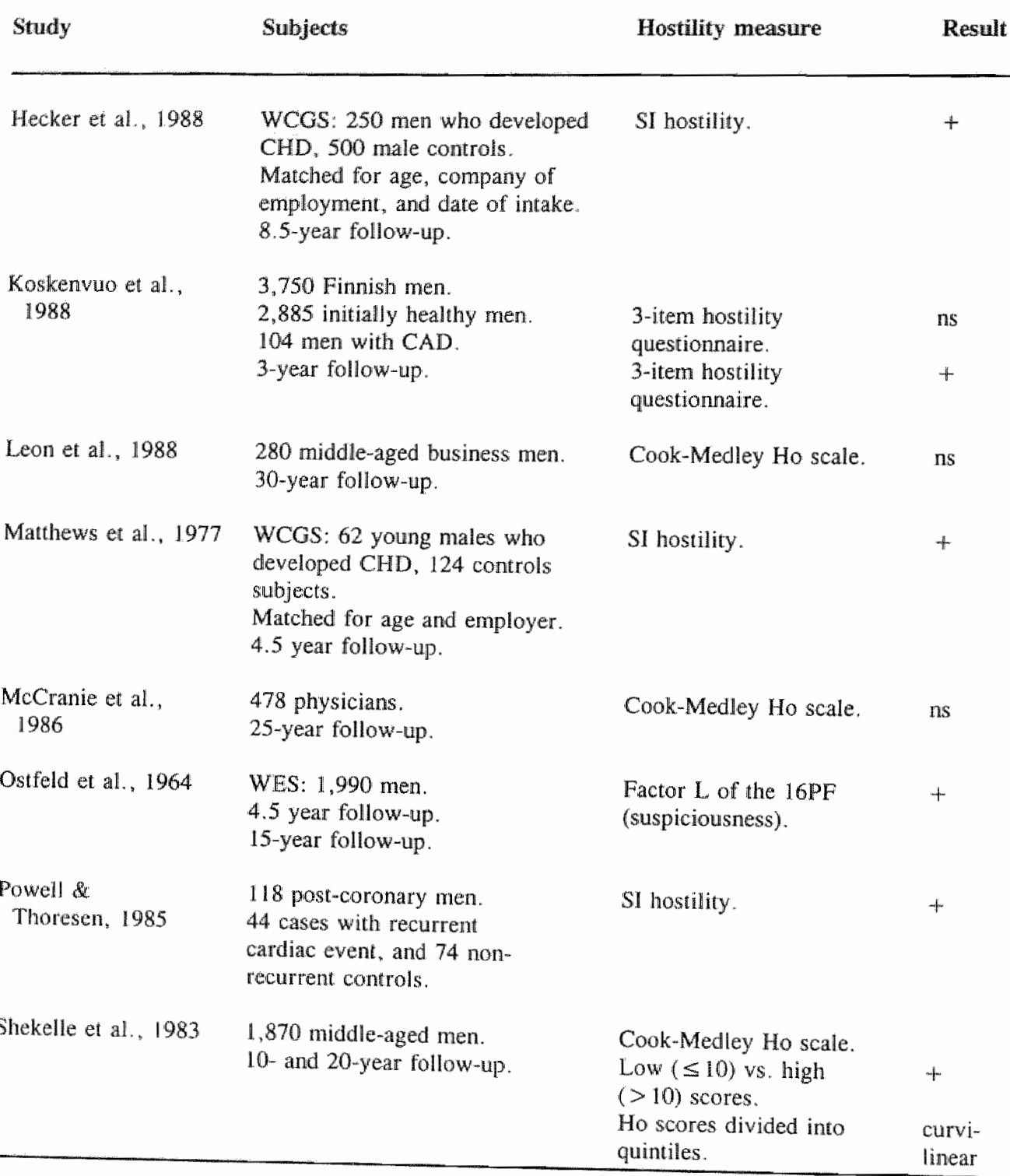




\section{Studies of initially healthy subjects}

\section{Self-report instruments}

To date, six prospective studies of the association between hostility and CHD have used the Ho scale. One of the first studies was published by Barefoot et al. (1983). who examined the relationship between hostility and health outcomes in a 25-year follow-up of 255 male medical students from the University of North Carolina who had taken the Minnesota Multiphasic Personality Inventory (MMPI) as part of their medical training. Subjects scoring above the median $(>13)$ showed a significantly higher incidence of coronary events (i.e. MI or CHD mortality) and total mortality than subjects with. Ho scores at or below the median ( $\leq 13)$. In a similar longitudinal study of 118 lawyers, Barefoot et al. (1989) found that high Ho scores were associated with poorer survival over a 28 -year follow-up period. A Composite Hostility (Chost, see section 1.1.4.1) score was found to be a better predictor of death than the full-scale Ho score. Increased risk of mortality was related to Cynicism, Hostile Affect, and Aggressive Responding, but not to Hostile Attribution, Social Avoidance, or Other. No information on gender was provided, so it is not clear whether gender differences influenced these results. Unlike the earlier study by Barefoot et al. (1983), which used both self-reported MI and angina pectoris and CHD mortality as endpoints, this study was based on the objective endpoint of mortality. Both studies used relatively small numbers of subjects, which resulted in small numbers of CHD patients during follow-up.

A large sample of 1,877 employed middle-aged men who participated in the Western Electric Study (WES) was evaluated by Shekelle et al. (1983). It was found that low Ho scores $(\leq 10)$ were associated with decreased incidence of major coronary events (i.e. MI or cardiac death) over a 10-year follow-up, even after adjustment for important CHD risk factors. In addition, analyses of 20 -year follow-up data showed that Ho scores were associated with increased risk of CHD mortality, malignant neoplasm mortality, and death from all other causes, again after controlling for standard risk factors. Note that in the analyses of both 10 -year and 20 -year follow-up data Ho scores were divided into quintiles. The results revealed a non-linear association, that is, incidence of CHD morbidity and mortality was highest in the middle quintile, which suggests a curvilinear relationship between hostility and cononary disease. More recently, Almada et al. (1991) reanalyzed data from the WES. Results of multivariate analyses indicated that scores on the Cynicism factor of the MMPI, which shows substantial overlap with the Ho scale, were independently related to coronary death and total mortality. MMPI-defined Neuroticism scores, by contrast, turned out to be unrelated to eicher endpoint.

Three studies have reported nonsignificant prospective associations between Ho scores and cardiovascular disease. A 25 -year follow-up study of 478 physicians with an average age of 47 at follow-up was conducted by McCranie, Watkins, Brandsma, and Sisson (1986). These physicians completed the MMPI during interviews for 
admission to the Medical College of Georgia. At follow-up, self-report information was collected with respect to history of angina pectoris and myocardial infarction. No significant association was found between Ho scores and 25-year CHD incidence (i.e. MI and CHD mortality) and total mortality. However, the fact that the MMPI was part of the selection procedure may have influenced the MMPI responses by social desirability and evaluation apprehension, which may explain the discrepancy between these findings and the results of two previous studies (Barefoot et al., 1983; Shekelle et al., 1983) at least partially. This is confirmed by the significantly lower Ho scores among students applying for admission to medical school than among students who were already enrolled, as in the study by Barefoot et all. (1983).

Leon, Finn, Murray, and Bailey (1988) prospectively evaluated the relationship between hostility and cardiovascular disease in a sample of 280 businessmen from Minnesota (mean age 45 years at baseline), who were followed for 30 years. Ho scores were not found to be predictive of the development of $\mathrm{CHD}$. However, the mean Ho score in this sample was low compared to average Ho scores reported in other studies (Barefoot et al., 1983; Shekelle et al., 1983). Therefore, social desirability bias as a possible explanation of these nonsignificant findings cannot be ruled out completely.

Bias due to social desirability influences does not seem to account for the negative findings reported by Hearn, Murray, and Luepker (1989). In a 33-year follow-up study of 1,399 male students (average age at follow-up 52) who entered the University of Minnesota in 1953, Ho scores failed to predict CHD morbidity, CHD mortality, and total mortality, either before or after adjustment for standard baseline risk factors. Additional analyses, based on the positive findings reported in other studies (Barefoot et al., 1983; Barefoot et al., 1987; Barefoot et al., 1989; Shekelle et al., 1983; Williams et al., 1980), did not reveal any significant relationships. Since the MMPI was taken as part of the freshman orientation and did not serve evaluative purposes, neither social desirability nor evaluation apprehension bringing about artificially low hostility scores are likely explanations of the lack of association. It should be noted that the subjects of this sample were roughly aged 19 when they completed the MMPI, which is considerably lower than the mean ages of subjects in several other studies. At this age, adult career and intimate relationships usually are not definitely established and personality characteristics may not be fully moulded yet. Thus, it is possible that the construct measured by the Ho scale at age 19 , is not quite comparable to the construct assessed 33 years later. This may have resulted in a nonsignificant association between hostility and CHD.

Three prospective studies employed other self-report measures to evaluate the association between hostility and cardiovascular disease. Haynes et al. (1980) investigated the relationship between a number of psychosocial variables and the development of $\mathrm{CHD}$ in 1,674 men and women (aged $45-77$ years) participating in the Framingham Heart Study between 1965 and 1967. These subjects were followed over an eight-year period. Clinical manifestations of CHD included in this study were $\mathrm{MI}$, sudden or nonsudden death, and angina pectoris with or without ECG deviations. An 
extensive psychosocial questionnaire, containing 20 psychosocial scales, was administered. Three of these scales are relevant to this review: Anger-in (suppression of anger), Anger-out (expression of anger), and Anger-discuss (a way of coping with feelings of anger). Analyses were done for males and fernales separately. Besides, both men and women were divided into three age strata: 45-54, 55-64, and $65+$. Female cases (aged 45-64) exhibited higher levels of Anger-in and Anger-discuss than females who remained free of CHD. Coronary males, especially in the 55-64 age group, showed significantly higher scores on Anger-in. Generally, the results of this large-scale longitudinal study suggest that the suppression of anger/hostility plays a role in the development of CHD in both men and women. It should be noticed that an extensive set of psychosocial variables was included in the statistical analyses in this study; only a few psychosocial aspects were found to be predictive of future CHD.

A Swedish sample of 795 middle-aged women, who were followed for 12 years, was studied by Hällström, Lapidus, Bengtsson, and Edström (1986). These women completed the Cesarec-Marke Personality Schedule, which also contains an aggression factor. The number of MI cases in the twelve-year period was 11 and that of coronary deaths 33 . The results of separate analyses of MI patients and coronary death patients indicated that aggression was unrelated to either MI or cardiac death. These findings do not correspond with the previous retrospective results of this population study, which demonstrated a positive association between aggression and MI (Bengtsson et al., 1973). These contrasting findings suggest that going through major life events, such as a serious disease (in this case, myocardial infarction), may affect personality traits like aggression.

Koskenvuo et al. (1988) evaluated the association between hostility and the incidence of CHD in the Finnish Twin Cohort, a three-year follow-up of 3,750 Finnish men aged 40-59. Hostility was assessed with a three-item self-report questionnaire reflecting irritability, anger proneness, and argumentativeness. In initially healthy men $(n=2,885)$, hostility did not predict CHD incidence. A follow-up period of three years may be too short to detect significant associations between hostility and cardiovascular disease.

As was already remarked in the section on conceptual issues (1.1.2), definitions of the hostility construct refer to negative attitudes toward and opinions about other people. Following this line of thought, two studies investigated the relationship between self-reported suspiciousness and adverse health outcomes. On the basis of data from the WES, Ostfeld, Lebovits, Shekelle, and Paul (1964) reported a positive association between suspiciousness and $4 \frac{1 / 2}{2}$ year incidence of CHD. Suspiciousness was measured by means of Factor L of Cattell's Sixteen Personality Factor Questionnaire (16PF), which is closely related to the Ho scale. A more recent study of 500 older adults ( 260 men and 240 women) also used Factor $L$ of the $16 \mathrm{PF}$ as a measure of suspiciousness (Barefoot et al., 1987). Subjects were 46-71 years of age at the outset of the study. Suspiciousness proved to be related to a higher mortality risk over a 15-year follow-up period. 
In sum, prospective studies of previously healthy subjects using paper-and-pencil measures of hostility have produced mixed results. Three studies found positive relationships (Barefoot et al., 1983, 1989; Haynes et al., 1980), one study reported an inverted U-shaped association (Shekelle et al., 1983), and five studies failed to find significant relationships (Hällström et al., 1986; Hearn et al., 1989; Koskenvuo et al., 1988; Leon et al., 1988; McCranie et al., 1986). Additionally, two studies which examined the closely related concept of suspiciousness yielded positive findings (Barefoot et al., 1987; Ostfeld et al., 1964). The outcomes of these studies may be biased due to social desirability factors (McCranie et al., 1986; Leon et al., 1988), low stability of hostility (Hearn et al., 1989), and small study samples (Barefoot et al., 1983, 1989).

\section{Behavioral ratings}

Two prospective CHD studies of initially healthy subjects used behavioral ratings of hostility. One of the first studies to carry out component analyses of structured interviews was conducted by Matthews et al. (1977). In this further analysis of data from the WCGS, which was already discussed above, a sample of 62 initially healthy men (aged 39-49 at intake) who developed clinical CHD (49 males with MI, 11 males with angina pectoris, and 2 males without specific diagnosis) during a $4 \frac{1}{2}$ year period of follow-up were compared with 124 age-matched healthy male controls on 44 separate ratings of interview responses. All behavioral measures were obtained at intake, when all participants were free of coronary disease. Among the variables significantly discriminating between cases and controls there were four hostility. related ratings: "potential for hostility" $(\mathrm{PH})$, "irritation at waiting in lines", "anger directed outward", and "experience of anger more than once a week". These results should be interpreted with caution, however, because data were analyzed on an individual item basis without any adaptation of levels of significance.

Also reanalyzing data from the WCGS, Hecker et al. (1988) examined 12 operationally defined components of the SI in 250 men who developed CHD (coronary death, MI, or angina pectoris) and 500 male control subjects, who were followed for $81 / 2$ years. Cases and controls were matched for age, company of employment, and date of intake. At intake, the mean age of the total sample was 48.5 years. Multivariate analyses, including all 12 interview components simultaneously in the model and adjusting for stanclard risk factors, indicated that hostility was the only component associated with increased risk of $\mathrm{CHD}$.

Altogether, the study by Matthews et. al (1977) as well as the study by Hecker and colleagues (1988) reported positive relationships between behavioral ratings of hostility and incidence of CHD. Note that these two studies should actually be considered as one study, as they both analyzed data from the WCGS. Furthermore, these positive findings are the opposite of the results of a number of previously reviewed longitudinal studies using self-report questionnaires of hostility that found no relation- 
ships (Hällström et al., 1986; Heam et al., 1989; Koskenvuo et al., 1988; Leon et al. 1988; McCranie et al., 1986).

\section{Studies of coronary patients and high-risk patients}

\section{Self-report instruments}

Koskenvuo et al. (1988) evaluated a subsample of 104 men from the previously discussed Finnish Twin Cohort with both self-reported hypertension and previous ischemic heart disease. It was found that a 3 -item hostility scale was associated positively with three-year incidence of CHD (cardiovascular mortality, death from all natural causes, and hospitalization).

\section{Behavioral ratings}

Powell and Thoresen (1985) studied the long-term prognosis after MI in a sample of 118 men with previously diagnosed infarction. These men were participants in the Recurrent Coronary Prevention Project, a 5-year clinical trial, aimed at the modification of Type A behavior in post-MI patients, and were followed for two years. By the end of this two-year follow-up, 44 men had suffered a recurrent cardiac event (MI or cardiovascular death). SI-defined hostility proved to be significantly associated with increased risk for recurrent MI. At the same time, however, patients with recurrent cardiac events were rated as having experienced more serious prior heart attacks.

Lastly, utilizing the Hostility Facet Scoring System for the rating of distinct Type A components, Dembroski et al. (1989) reanalyzed audiotaped interviews of participants in the MRFIT study. One hundred and ninety-two high-risk men with subsequent non-fatal MI or sudden death and 384 matched controls were compared with respect to $\mathrm{PH}$ and its three subcomponents (hostile content, intenstity of hostility, and hostile style) and various other Type A fearures. Only overall ratings of PH, when dichotomized into "low" and "high" categories, and stylistic hostility proved to be significantly predictive of CHD (coronary death or MI) after adjustment for traditional risk factors. Additional analyses indicated, however, that this association was limited to the younger subjects ( $\leq 47$ years) in the sample.

In conclusion, three studies of coronary patients or patients at high risk for CHD found positive predictive associations between self-reported hostility (Koskenvuo et al., 1988), or Sl-derived hostility (Dembroski et al., 1989; Powell \& Thoresen, 1985) and recurrent CHD.

\section{Prospective studies: Summary and conclusions}

In all, 16 prospective studies concerning the association between hostility and CHD have been reviewed. Twelve studies reported positive findings and four studies failed 
to find significant relationships. Although there are a number of studies with negative findings, the majority of studies clearly demonstrated a strong and consistent association between hostility and subsequent development of CHD. The inconsistency of results may partly be due to differences in methods used for the assessment of hostility. All longitudinal studies that used the SI to measure hostility yielded positive associations. In contrast, studies employing the Ho scale or other self-report questionnaires obtained mixed results. Nevertheless, empirical evidence so far lends support to the basic assumption that hostile people are at increased risk for the development of cardiovascular disease.

\subsubsection{Potential mechanisms linking hostility and health}

Evidence of an association between the personality traits of anger and hostility and subsequent cardiovascular disease and other adverse health outcomes raises the question of potential mechanisms: which processes might provide explanations for the connection between these psychological concepts and disease? Several models have been proposed, which will only be outlined here in view of the objectives of this thesis (for an extensive review see Suls \& Rittenhouse, 1990).

\section{Psychophysiological reactivity model}

This model represents, perhaps, the most popular explanation of the association between anger/hostility and CHD. It is hypothesized that individuals with a disposition to be hostile or angry exhibit exaggerated levels of neuroendocrine and hemodynamic responsiveness to stressors in comparison with individuals low in hostility and anger (Williams et al., 1985). Hostile persons are supposed to experience anger more frequently and intensely than nonhostile people. Owing to their distrust, hostile people may be watchful in interpersonal interactions more frequently, scanning their social environment for signs of possible ill-treatment. This inclination for anger experience and watchful observation of other people's behavior is assumed to bring about increased psychophysiological reactivity. This psychophysiological reactivity (e.g. blood pressure, heart rate, and catecholamines) is considered to promote the development of cardiovascular disease and to accelerate the symptoms of CHD (e.g. MI and angina pectoris) by increasing endothelial injury and subsequent accumulation of atheroma (Krantz \& Manuck, 1984). Recent reviews of reactivity studies indicate that evidence of a relationship between hostility and cardiovascular reactivity is mixed and does not allow of firm conclusions (Houston, 1994; Manuck, Kaplan, Muldoon, Adams, \& Clarkson, 1991; Smith, 1992; Smith \& Christensen, 1992; Suls \& Wan, 1993). Suls and Wan (1993) performed a series of meta-analyses which indicated that hostility, as assessed by various measures (primarily the Ho scale, the $\mathrm{BDHI}$, and the $\mathrm{SI}$ ), was not consistently associated with blood pressure and heart rate reactivity in studies using traditional (nonsocial) stressors (i.e. mental arithmetic, cold pressor, Stroop color-word task). However, a distinction between provocative stress- 
ors and nonprovocative stressors, revealed that Potential for Hostility was related to increased systolic and diastolic blood pressure responses and Ho scores were associated with diastolic blood pressure responses to stressors of a provocative nature. These results clearly suggest that the use of adequate interpersonal stressors may be a fruitful avenue for future studies of the psychophysiological reactivity hypothesis.

\section{Psychosocial wulnerability model}

This view of the connection between hostility and disease emphasizes the importance of psychosocial factors. On the basis of the description of hostile and angry people given above, it is supposed that the social environment of these persons is relatively unfavorable. Several studies have provided empirical evidence of this hypothesis. For instance, it was found that high Ho scores were associated with low levels of social support, high levels of interpersonal conflict, experience of more frequent and serious everyday quarrels, and reports of more negative life events (Barefoot et al., 1983; Hardy \& Smith, 1988; Houston \& Kelly, 1989; Scherwitz, Perkins, Chesney, \& Hughes, 1991; Smith \& Frohm, 1985; Smith et al., 1988). Together, these results suggest the presence of a constellation of negative psychosocial characteristics which may lead to an increased risk of development of disease (Krantz, Contrada, Hill, \& Friedler, 1988; Syme, 1987).

These findings are in line with the basic psychosocial vulnerability hypothesis. Nevertheless, firm conclusions should be avoided because this line of research has several shortcomings. Firstly, studies of the psychosocial correlates of hostility have mainly utilized the Ho scale, so that knowledge regarding the psychosocial correlates of other hostility measures is still lacking. Secondly, there may be a relationship between these psychosocial correlates of hostility and the personality trait of neuroticism; they may overlap. Thirdly, because item content of hostility measures and psychosocial scales are possibly to some degree similar, reported correlations may have been heightened artificially. These drawbacks call for further research including relatives" ratings of everyday stresses for hostile persons.

\section{Transactional model}

The transactional approach is an extension and a blend of the two previously described models of underlying mechanisms (Smith \& Pope, 1990; Suls \& Sanders, 1989). The psychophysiological model focuses mainly on reactions associated with hostility and anger, and the psychosocial model concentrates particularly on the psychosocial correlates of these concepts. The transactional perspective, however, also emphasizes the effects of hostility and anger on the individual's social environment. From this point of view, anger and hostility are considered stress-provoking, i.e. angry and hostile individuals are considered active creators of an antagonistic, unfavorable social environment. As was indicated above, hostile persons are inclined to expect the worst from other people. This, in turn, may lead them to behave in an 
antagonistic way. Such antagonistic behavior probably evokes unpleasant, unfriendly responses from their social environment, which supports or even reinforces initial. negative expectations and beliefs. This may lead to a pattern of escalating, reciprocal antagonism. In the transactional approach, personal characteristics and the social context are taken to be interdependent (Bandura, 1977). Research in the domain of social psychology has produced evidence of such expectancy confirmation processes occurring in social interactions (Darley \& Fazio, 1980; Snyder \& Swann, 1978). Therefore, it should not come as a surprise that hostile persons are more involved in interpersonal conflicts and perceive less social support than non-hostile persons. Moreover, besides supporting a negative, mistrustful, and cynical outlook, this social context would also contribute to chronic physiological (hyper)reactivity.

The findings of several studies discussed earlier with respect to the psychophysiological reactivity and psychosocial vulnerability approaches, are in line with this transactional perspective. To date, however, direct empirical tests of the separate elements of the transactional model have not been carried out.

\section{Health behavior model}

A fourth model concerning the association between hostility and disease is based on health behavior associated with hostility. The three previous models of underlying. mechanisms all suppose that physiological reactivity due to stressors may explain the link between hostility and health and disease. Leiker and Hailey (1988) suggested that the poor health habits of highly hostile individuals may be an alternative explanation of the association between hostility and disease. In a sample of 202 undergraduate students, they found that high Ho scores were related to less self-reported fitness training (e.g, aerobics), less self-care (e.g. sufficient sleep and dental hygiene), and a higher alcohol intake. In a related study, Houston \& Vavak (1991) showed that persons with high Ho scores tended to drink more alcohol, to drive more frequently after drinking, and also to have greater body mass index values. Several other studies also reported significant associations between hostility and smoking and alcohol consumption (e.g. Koskenvuo et al., 1988; Scherwitz et al., 1992; Siegler, Peterson, Barefoot, \& Williams, 1992). In addition to reported evidence of an association between hostility and standard risk factors such as smoking, alcohol intake, and lack of physical exercise, it has recently been suggested that hostility might be related to other thigh-risk behaviors. For instance, Suls and Sanders (1989) reported that hostile people may postpone seeking medical treatment, and Lee et al. (1992) demonstrated that hostile persons are less inclined to adhere to prescribed medical treatment, thereby contributing to increased health risk. Note that these behaviors may be lifethreatening in the case of a person with an impending heart attack. 


\section{Biological vulnerability model}

Several authors have proposed a model which is quite different from all previously discussed mechanisms (Krantz \& Durel, 1983; Suls \& Sanders, 1989). It is postulated that it is an underlying structural or constitutional weakness which is causally related to health and disease, rather than the personality dimensions of hostility and anger themselves. From this biological vulnerability perspective, individual manifestations of anger, hostility, and aggression are considered to be caused by fundamental individual biological differences. These individual biological differences may also confer risk of disease. Both personality characteristics, such as hostility and anger, and disease are considered to be consequences of underlying biological factors (e.g. physiological reactivity) (see Kaplan, Botchin, \& Manuck, 1994; Williams, 1994).

\section{Conclusion}

The above overview of potential mechanisms linking hostility and disease clearly indicates that the models that have been described are not mutually exclusive. On the contrary, the psychophysiological reactivity, psychosocial vulnerability, and transactional approaches have much in common. Therefore, they may be considered complementary rather than contradictory. Furthermore, although it provides an alternative explanation for the connection between hostility and disease, the health behavior model is not incompatible with the other mechanisms. Lastly, even the biological vulnerability model is not entirely irreconcilable with the preceding models. For instance, given that hostility and anger are biologically determined, people exhibiting high levels of these characteristics probably produce negative social environments. Consequently, hostile individuals are more likely to be exposed to social contexts that are taxing, particularly for them.

\subsubsection{Developmental antecedents of hostility}

In recent years, research in the field of hostility and health has also paid attention to developmental factors that may foster hostility. At present, evidence with respect to early determinants of hostility is limited. However, studies providing insight into the process of the development of individual differences in hostility would be important from the perspective of prevention and intervention. In this section on the childhood origins of hostility, studies focusing on genetic factors will be reviewed first, followed by studies addressing the possible influence of socialization processes.

\subsubsection{Familial aggregation of hostility}

In the past few years, several twin studies have sought to assess the potential genetic foundations of hostility. Reanalysis of a sample of 80 monozygotic (MZ) and 80 dizygotic (DZ) male twins from the California Type A Twin Study showed that $\mathrm{PH}$ 
ratings were heritable (Matthews, Rosenman, Dembroski, Harris, \& MacDougall, 1984). In another sample, significant evidence of a hereditary component for Ho scores was found (Camelli, Rosenman, \& Swan, 1988). Smith, McGonigle, Turner, Ford, and Slattery (1991) performed a similar twin study, which provided significant heritability estimates for the total Ho scale. In addition, results of a study by Rose (1988) suggested the presence of a genetic influence on the subset of Ho scale items constituting the Cynicism factor of the MMPI. On the other hand, a study of about 600 Swedish twin pairs found that individual differences in scores on the Ho scalle were mainly due to nonshared environmental effects (Pedersen et al. , 1989). Besides, a recent study carried out by Matthews and colleagues (1992) revealed little similarity in hostility between parents and offspring in a sample of 142 upper middle-class families.

In summary, evidence produced by research in the field of behavioral genetics is not particular strong nor quite consistent. Nonetheless, the findings that are available suggest that genetic factors do, at least to some extent, contribute to the development of hostility. It should be noted that these results certainly do not imply that psychosocial factors are less influential in the development of hostility. On the contrary, current views in the domain of developmental psychology underline the reciprocity between genetically based psychological characteristics on the one hand and environmental factors on the other (Plomin \& Bergeman, 1991; Scarr \& McCartney, 1983). For instance, a child's inclination to react in a mistrustful, irritable, angry, or aggressive manner is likely to generate negative or even more antagonistic and rejecting responses from its parents. Such parental behavior will, in turn, be conducive to the further development of hostility.

\subsubsection{The impact of parental behaviors on hostility}

As was indicated before, the early family environment is likely to play an important role in the expression of hostility, even if this psychosocial characteristic should have a genetic basis (see e.g. Matthews \& Woodall, 1988). To date, a limited number of studies examining the influence of parental behaviors and attitudes on the etiology of hostile characteristics have appeared. In a study of male and female undergraduate students, high Ho scores were found to be associated with ratings of families of origin as high in conflict and low in cohesion and support (Smith et al., 1988). In a similar study of undergraduates by Houston and Vavak (1991), subjects were requested to give their childhood recollections of their parents' behaviors. Results indicated that high Ho scores were associated with descriptions of parents as having been less sincerely accepting, more interfering in the subject's desires and wishes as a child, and more punitive. In addition to these findings, Woodall and Matthews (1989) observed that children with high scores on an adapted version of the Ho scale were more likely to come from families described by their parents as low in supportiveness and interpersonal involvement. 
Although the number of relevant studies is small, the available evidence supports the hypothesis that the development of hostility is facilitated in family contexts that are characterized by low degrees of positive inwolvement and support and high degrees of antagonistic interaction. However, caution is warranted when interpreting. these studies of potential parental influences on hostility, as several twin studies have demonstrated that genetic factors may affect retrospective reports of parental behavior. For example, the findings of a study by Plomin, McClearn, Pedersen, Nesselroade, and Bergeman (1988) suggest that the influence of genetic factors on the recollection and perception of former as well as current parental conduct is affected by the reciprocal processes discussed earlier. However, a recent study of monozygotic (MZ) twins suggests that the association between hostility and perceived parental rearing behavior is not entirely determined by genetic factors. In a sample of 25 adult male $\mathrm{MZ}$ twin pairs, high levels of hostile control and low levels of positive involvement of parents were found to be associated with high levels of self-reported hostility (as defined by the Ho scale). In order to rule out potentia] genetic influences on this observed covariation between retrospectively reported parental behavior and hostility, co-twin difference scores for hostility and perceived parental behavior were correlated. The results showed that the more hostile $\mathrm{MZ}$ co-twin described his parents as displaying a greater degree of hostile control (McGonigle, Smith, Benjamin, \& Turner, 1993), which means that nongenetic variance in hostility was associated with perceived differences in early environments.

Available research results indicate that individual differences in hostility may be influenced by genetic and family environment factors. This conclusion is tentative, however. There is an obvious need for additional prospective investigation of the childhood antecedents of hostility to gain a better insight into the determinants of early risk for disease. In this respect, a first promising effort has been made by Woodall and Matthews (1993). In a sample of 108 boys and girls (aged 10-18) who were followed for 4 years, hostility was significantly associated with family environment (i.e. family support). These results show a striking correspondence with findings obtained by studies using adult samples and make out a strong case for further study of the etiological antecedents of hostility.

\subsection{VITAL EXHAUSTION AND CORONARY HEART DISEASE}

During the last decade, research on the association between feelings of exhaustion and coronary heart disease (CHD) has accumulated. The present section begins with a concise discussion of the origins of the concept of "vital exhaustion" (VE). The two following sections present a review of both prospective and cross-sectional evidence concerning the association between VE and CHD. Next, two important issues will be scrutinized; the relationship between $\mathrm{VE}$ and depression and the possibillity that VE is a marker of subclinical heart disease. In the final section, the need for the development of a new instrument to measure $V E$ is described briefly. 


\subsubsection{Vital exhaustion as a precursor of myocardial infarction}

Feelings of excess fatigue and general malaise have repeatedly been found to be among the most prevalent premonitory symptoms of myocardial infarction (MI) and sudden cardiac death (SCD). Percentages of people who experienced extraordinary tiredness or lack of energy prior to $\mathrm{MI}$ or SCD vary from $13 \%$ to $70 \%$ (Alonzo, Simon, \& Feinleib, 1975; Fraser, 1978; Gillum, Feinleib, Margolis, Fabsitz, \& Brasch, 1976; Kinlen, 1973; Klaeboe, Otterstad, Winsnes, \& Espeland, 1987; Kuller, 1978; Kuller, Cooper, \& Perper, 1972; Rissanen, Romo, \& Siltanen, 1978; Simon, Feinleib, \& Thompson, 1972; Stowers \& Short, 1970; Thiele, Simon, \& Thiele, 1985). One of the explanations for this wide range is that there is disagreement about the symptoms that should be covered by the questions asked to coronary patients.

Guided by the model of the General Adaptation Syndrome described by Selye (1977), Appels and his colleagues assumed that prolonged stress may result in a state of exhaustion preceding MI. Because of the above-mentioned absence of a clear picture of the mental precursors of MI, it was decided to interview a large number of coronary patients and their spouses, asking them about the feelings and behavioral alterations experienced in the year prior to the coronary event. On the basis of these interviews, the pre-coronary mental state was labeled "vital exhaustion" (VE). VE has three core characteristics: 1) unusual fatigue and lack of energy, 2) increased irritability and 3) feelings of demoralization (Appels et al., 1994).

The responses of the coronary patients during the interviews were used to construct an item pool, comprising sixty-three questions. Next, this initial scale was used in two case-control studies (Appels, 1980; Verhagen, Nass, Appels, van Bastelaer, \& Winnubst, 1980). Thirty-seven items discriminated between coronary patients and healthy control subjects. These items were given the prefix "form $A$ " of the Maastricht Questionnaire (MQ-A).

Subsequently, several prospective and case-control studies were carried out to investigate the strength of the association between VE and MI. The results of these studies will be presented below.

\subsubsection{Evidence from prospective studies}

A prospective sudy was launched in 1979, when the Municipal Health Authority of Rotterdam (The Netherlands), initiated a health check-up of city employees. This study was called the Rotterdam Civil Servants Study (RCSS). Between January 1979 and December $1980,3,877$ male employees, aged between 39 and 65 , were screened. The physical examination consisted of measurements of blood pressure, serum cholesterol, glucose tolerance, relative weight, smoking and angina pectoris as measured by the Rose Questionnaire (Rose, Blackburn, Gillum, \& Prineas, 1982). A resting electrocardiogram was also included in the cardiovascular screening. All participants completed form A of the MQ (37 items) as well as 21 new items, derived from a new series of clinical interviews. 
The average period of follow-up was 4.2 years. During this interval, 21 males who were free of CHD at screening, died of $\mathrm{MI}$, and 38 subjects suffered a welldocumented non-fatal MI. Subjects were classified "exhausted" if the MQ-A score was above the median. The relative risk of exhaustion for a fatal or non-fatal Ml among those free of CHD at screening proved to be $2.67(\mathrm{p}<0.01)$, adjusted for age, cholesterol, smoking and blood pressure. Further examination of this association, taking length of time interval into account, revealed a sharp decrease in the relative risk of events occurring in the first $(10.05)$, second (2.23), third (3.04) or fourth year of follow-up (0.68). This finding strongly suggests that VE is a short-term risk indicator of MI. No relationship emerged between elevated MQ-A scores at screening and future cancer or gastrointestinal illness, which suggests that $V E$ is specifically related to future CHD (Appels \& Mulder, 1988; Appels \& Schouten, 1991).

To design a definitive questionnaire, item analysis was performed. Sixteen out of the 37 items of the MQ-A contributed to the predictive power of the questionnaire. Furthermore, 8 out of the additional 21 items were predictive. By selecting the predictive items the final form of the MQ (form B) was constructed. Because of their dubious content validity, three items were removed, resulting in a final form (form B) consisting of 21 items (Appels, Höppener, \& Mulder, 1987).

Since there is very little prospective evidence of an association between VE and coronary disease, it was worth investigating whether the database of the Kaunas Rotterdam Intervention Study (KRIS), a 9.5 year follow-up study (Appels \& Otten, 1992), could provide evidence to support such an association. In the KRIS, 3,365 men took part in a cardiovasular screening program between 1972 and 1974 . All subjects filled out the Reeder Stress Scale at the beginning of the program. Although this scale is outdated now, it contains one item which resembles the concept of VE quite closely: "I am completely exhausted mentally and physically at the end of the day". Of the participants who were free of CHD at screening 69 died of ML. It was found that the cumulative incidence of cardiac death was $45 / 1000$ in the exhausted group, $30 / 1000$ in the intermediate group, and 26/1000 in the non-exhausted group. Subjects who felt exhausted at screening appeared to be most at risk cluring the initial phase of the follow-up period. The hazard ratios for exhaustion were $8.96,6.33$, 4.47 and 3.16 for the first $10,20,30$ and 40 months of follow-up, respectively. After this, the association was no longer statistically significant (Appels \& Otten, 1992). These findings again support the hypothesis that feelings of exhaustion are associated with an increased risk of cardiac death in men who are free of CHD.

The results of a study by Siegrist, Peter, Junge, Cremer, and Seidel (1990), who followed 416 blue-collar workers, aged between 25 and 55 , for 6.5 years, are in agreement with the other prospective studies discussed above. They found that a state of exhaustive coping, reflected by frustrated but persistent efforts and associated negative affects like disproportionate irritability and the incapacity to withdraw from work obligations, was strongly associated with the incidence of $\mathrm{MI}$, even after controlling for all standard risk factors (Siegrist et al., 1990). 
In conclusion, these prospective studies all lend support to the notion that feelings of exhaustion precede manifest heart disease in apparenly healthy males. However. these sudies did not rule out the possibility that a state of VE is predictive of MI because in reflects subclinical CHD. Particularly the fact that feelings of exhaustion seem to be a relatively short-term risk indicator raises the question whether VE reflects subclinical heart disease. This issue will be addressed in more detail in a later section.

\subsubsection{Evidence from case-control studies}

The most comprehensive case-control study of the association between VE and MI was carried out by Falger (1989). In this study, 133 male MI-cases were compared with 133 neighborhood controls and 192 hospital controls. The latter reference group was included to control for any possible effects of hospitalization on the recall of feelings of exhaustion. Cases and controls were divided into exhausted and nonexhausted subjects on the basis of a score above or below the median of the MQ (that is, eight affirmative replies or more to the 21 questions). Employing this cut-off point, Falger found an estimated relative risk of first MI associated with VE of 7.35 (95\% CI 4.3 - 13.3) in the neighborhood control group and 2.90 (95\% CI $1.7-4.9$ ) in the hospital control group. These results do not only indicate that feelings of exhaustion constitute a risk indicator of $\mathrm{MI}$, but that these feelings can also be observed in other patient groups. This finding may be interpreted as follows: (1) VE is (also) a precursor of diseases other than CHD; (2) other diseases are related to one or more elements of VE (e.g. sleep problems or pain); or (3) recent hospitalization may have affected the findings due to retrospective bias (Falger, 1989). The findings of a study performed by Nelissen-de Vos suggest that the second explanation of Falger's study is the most plausible. Painful ailments may cause somewhat elevated MQ scores (Nelissen-de Vos, 1994).

The question whether the association between VE and first MI reported by Falger can also be observed in women, was investigated in a study comparing 79 women hospitalized because of a first MI with 90 women hospitalized in the General Surgery and Ortopedic Surgery units of two regional hospitals. It was found that MI-cases reported significantly more symptoms of VE than control subjects. The estimated relative risk of exhaustion associated with $\mathrm{MI}$, controlled for age, hypertension, smoking, and non-amginal pain, was $2.75(95 \%$ CI 1.3 - 5.9) (Appels, Falger, \& Schouten, 1993). Therefore, the conclusion that VE is a risk indicator of MI in both genders is warranted.

Because initially all research on VE was carried out in the Netherlands, Mendes de Leon (1988) conducted a case-control study of a group of patients admitted to John Sealy Hospital, Galweston, Texas (USA), in order to cross-validate the Dutch findings. Twenty-two male patients with unstable angina pectoris (cases) were compared with 44 hospital controls, admitted for relatively acute conditions (mainly orthopedic patients). The observed odds ratio, adjusted for age, education, marital status, and 
smoking proved to be 2.36 (95\% $\mathrm{CI} 0.95-5.88 ; \mathrm{p}<0.07$ ), and thus lent support to the relationship between VE and manifest coronary artery disease (Appeis \& Mendes de Leon, 1989).

In summary, all case-control studies reviewed here clearly suggest that a state of $\mathrm{VE}$ is a precursor of CHD. Given the retrospective nature of the case-control design, the possibility of retrospective bias and some confounding due to somatic non-cardiac complaints, camnot be ruled out completely. However, it is unlikely that these factors sufficiently account for the relationship between feelings of exhaustion and future manifestations of CHD.

From the outset of the investigations into the mental precursors of MI two questions have been of major importance: (1) Does the constellation of symptoms preceding MI reflect a depression? and (2) Are these feelings of exhaustion a subclinical marker of CHD itself?

\subsubsection{Vital exhaustion and depression}

Many symptoms reported by cardiac patients describing their mental state prior to MI (for example, "I already felt tired already on waking up" or "I had a feeling of hopelessness") are also used as criteria for the diagnosis of depression. However, there is reason to believe that VE should be distinguished from depression. The core feeling of a state of exhaustion is constituted by feelings of depletion of energy, represented by items such as: "I feel weak all over" or "My body is like a battery that is losing its power". Such feelings may lead to feelings of depression because of their debilitating effects. VE and depression share a number of symptoms like fatigue, waking up exhausted and irritability. However, mood disturbances, loss of selfesteem and feelings of guilt are usually absent from the reports of exhausted subjects.

Empirical evidence of a distinction between VE and depression was provided by a study by van Diest and Appels (1991). They employed the Profile of Moods State (POMS) to monitor feelings of a depressed mood in 12 exhausted and 10 non-exhausted males. Excess fatigue and loss of vigor discriminated exhausted subjects from non-exhausted subjects. Depressed mood, however, the key symptom of depressive disorders, was hardly ever mentioned by the exhausted individuals. In addition, current affective, cognitive, motivational and somatic symptoms of depression were measured with the Beck Depression Inventory (BDI), which yielded similar results. The symptoms occurring most frequent in the exhausted group appeared to be: fatigability, work inhibition, sleep disturbances, and loss of libido. Again, a depressed mood was hardly ever mentioned. All participants in this study were allso interviewed by two skilled psychiatrists. None of them was diagnosed depressed (van Diest \& Appels, 1991). These findings strongly suggest that the variance shared by the MQ, used to measure VE, and questionnaires assessing depression is attributable to common somatic and vegetative symptoms, whereas the unique variance of the depression inventories can be attributed to questions asking about a depressed affect 
and cognition. Therefore, it is likely that most depressive individuals are exhausted but that only a minority of exhausted persons are clinically depressed.

\subsubsection{Vital exhaustion: a marker of subclinical heart disease?}

To test the hypothesis that VE reflects subclinical heart disease, Kop performed a prospective study of 127 consecutive Percutaneous Transluminal Coronary Angioplasty (PTCA) patients. To be more specific, he investigated the association between VE (as measured by form B of the MQ) and the extent of atherosclerosis, the changes in MQ scores before and after PTCA, and the clinical course after successful angioplasty among both exhausted and non-exhausted patients. The incidence of new cardiac events was registered during a 1.5 year follow-up. Results showed a significant relationship between the number of diseased vessels and MQ scores both before and after treatment. The explained variance, however, was of low magnitude $(4 \%)$. VE and left ventricular ejection fraction appeared to be unrelated (Kop, Appels, Mendes de Leon, de Swart, \& Bär, 1994). Furthermore, it was found that VE was predictive of new coronary events occurring within the 18 months of follow-up, controlling for the extent of coronary artery disease and left ventricular ejection fraction, smoking, diabetes mellitus, and previous MI (Kop et al., 1994). These findings strengthened the idea that VE might be considered to be an independent risk indicator of CHD. Definitive conclusions will have to be postponed, however, because, as yet, little is known about the possible biological mechanisms underlying the association between VE and CHD. Kop (1994) found that the fibrinolytic system is impaired in exhausted men, which was indicated by elevated levels of plasminogen activator inhibitor, (PAI-1). Furthermore, a decreased amount of slow-wave sleep was observed in exhausted males by van Diest and Appels (1994). Current views on the pathogenesis of coronary artery disease and the acute coronary syndromes, as presented by Ross (1993) and Fuster, Badimon, Badimon, and Chesebro (1992), raise the question whether psychoneuroimmunological factors may play a role in the pathogenesis of $\mathrm{CAD}$. Guided by these ideas it could be theorized that $\mathrm{VE}$ is related to functional processes in the coronary vessels, especially the release of cytokines induced by inflammation of coronary arteries in susceptible persons.

\subsubsection{Conclusion}

Although some questions still remain to be solved, the present-day body of evidence clearly indicates that VE is a risk indicator of manifest heart disease. To measure the state of exhaustion, all of the studies reviewed for this thesis used the Maastricht Questionnaire. The MQ is shown to have predictive validity in two prospective studies (Appels \& Mulder, 1988; Kop et al., 1994) and discriminates well between coronary cases and control subjects (Appels et al., 1993; Falger, 1989). At the same time, however, it also has some major drawbacks. For example, elevated scores on self-report questionnaires asking about somatic and psychological symptoms are often 
due to a general tendency to complain (Costa et al., 1985). This may have resulted in an unknown number of false positives. Furthermore, the assessment cannot be corrected if a subject misinterprets a question, nor does it give the opportunity to record idiosyncratic expressions. Lastly, a questionnaire is not really the appropriate instrument for recording important qualitative information. Hence its use as a screening device for the selection of subjects for laboratory and clinical investigations is disputable. This was further underscored by van Diest in the preparation of an experimental. study of the sleep physiological characteristics of exhausted and non-exhausted men. Carefully selected samples were needed for this study. For this reason, several experts were requested to interview his questionnaire-based samples of 15 exhausted and 10 non-exhausted males. Two out of the 15 exhausted men turned out to have a serious somatic disease, three were considered not representative due to the chronicity of their symptoms, and one subject proved to be non-exhausted (van Diest \& Appels, 1994). Mainly on the basis of van Diest's experiences, it was decided to construct an interview to measure the mental precursors of $\mathrm{NI}$ in order to overcome the disadvantages inherent in the questionnaire method of assessment. One of the aims of the study presented in this thesis was to develop such an interview. Chapters 6 and 7 describe the development of this interview and discuss data on its reliability and validity.

\subsection{DESIGN OF THE THESIS}

The main aim of the present thesis is to investigate the association between hostility, vital exhaustion and myocardial infarction. A case-control study was carried out to explore these (inter) relationships. Cases were 81 males (mean age 55.6 years; sd $6.8)$, hospitalized because of a first clinically documented myocardial infarction (MI), who were compared with 168 neighborhood controls (mean age 54.9 years; sd 6.9).

Chapters 2 and 3 examine the relationship between hostility and myocardial infarction. In Chapter 2, the risk of cynical hostility, measured with the Cook and Medley Hostility (Ho) Scale, is investigated. The risk of hostility, indexed by the Buss-Durkee Hostility Inventory (BDHI), is explored in chapter 3.

Chapters 4 and 5 deal with studies exploring the developmental antecedents of hostility. In chapter 4 the relationship between perceived parental rearing behavior and individual differences in hostility in a sample of young adults (both males and females) is addressed, while the results of a test concerning the relationship between perceived parental rearing behavior and hostility using the case-control database are discussed in chapter 5 .

Chapters 6 and 7 deal with the development of a new instrument to assess the mental precursors of MI. The development of an interview to measure feelings of vital exhaustion and a comparison with the Maastricht Questionnaire (MQ) is described in chapter 6 . Chapter 7 presents data on the reliability and validity of the interview. 
Chapter 1

Chapter 8 describes the association between hostility and vital exhaustion and the risk of MI associated with each of these factors when included simultaneously in a multivariate analysis.

Finally, in chapter 9, the findings of the study and implications for future research are discussed. 


\section{Chapter 2}

\section{HOSTILITY AND MYOCARDIAL INFARCTION IN MEN $^{1}$}

\subsection{SUMMARY}

This case-control study examines the association of hostility (as measured by the CookMedley Hostility Scale) with myocardial infarction in adult males from The Netherlands. Subjects included patients with first myocardial infarction (MI; $N=81$ ), who were compared with a neighborhood control $(\mathrm{NC})$ group $(\mathrm{N}=168)$. Cases had somewhat higher scores on the Cook-Medley Hostility Scale, although the differences between cases and controls were not statistically significant. Multivariate analysis revealed the presence of an interaction between hostility and age, suggesting that the association of hostility with MI is age-dependent. The results indicate that hostility only constitutes a risk indicator for first $\mathrm{MI}$ in men who are younger than 50 years of age.

\subsection{INTRODUCTION}

The last three decades have yielded numerous investigations on possible psychosocial risk factors of coronary heart disease (CHD) (Williams \& Barefoot, 1988). More recently, studies have increasingly directed their attention to hostility as a potential predictor of CHD (Smith, 1992). The relationship between hostility and CHD has been examined extensively in both cross-sectional and prospective studies, using ratings based on the Type A structured interview (Dembroski \& Costa, 1987; Hecker, Chesney, Black, \& Frautschi, 1988) and/or self-report "paper-and-pencil" tests, in particular the Cook-Medley (Cook \& Medley, 1954) Hostility Scale, which was originally derived from the MMPI.

One of the first cross-sectional studies was carried out by Williams and his colleagues (Williams et al., 1980). Both Type A behavior and Cook-Medley scores were found to be significantly related to the severity of coronary atherosclerosis. This initial study was followed by several studies of angiography patients that failed to replicate the association between Cook-Medley scores and coronary artery disease (CAD) (Dembroski, MacDougall, Williams, Haney, \& Blumenthal, 1985; Helmer,

\footnotetext{
${ }^{1}$ Meesters, C.M.G., \& Smulders, J. (1994). Hostility and myocardial infarction in men. Jownal of Psychosomatic Research, 38, 727-734.
} 
Ragland, \& Syme, 1991). Two other case-control studies found no differences in the average Cook-Medley scores beween CHD patients and controls having no history of CHD (Friedman \& Booth-Kewley, 1987; Fontana et al., 1989).

Structured interview (SI) hostility ratings were employed in two studies by Dembroski and his colleagues (Dembroski et al., 1985; MacDougall, Dembroski, Dimsdale, \& Hackett, 1985), which indicated that hostility was significantly associated with CAD. In the study by Helmer et al. (1991), no association was reported between SI-defined hostility and severity of $\mathrm{CAD}$, whereas the recent work of Barefoot et al. suggests that hostility ratings from their interview rating system are associated with severity of CAD (Barefoot, 1992).

An important finding was reported by Siegman and colleagues, who used the BussDurkee Hostility Inventory (BDHI) to assess hostility in a study of 72 angiography patients (Siegman, Dembroski, \& Ringel, 1987). They factor analyzed the BDHI, yielding an experiential and an expressive factor. Only the expressive factor proved to be significantly associated with severity of coronary atherosclerosis, and this effect was limited to patients below 60 years of age. In the same age group, the experiential factor was negatively correlated with atherosclerosis. Neither factor showed any effect among patients above 60 years of age.

Two prospective studies of CHD have made use of interview ratings of hostility. In the Western Collaborative Group Study (WCGS) as well as in the Multiple Risk Factor Intervention Trial (MRFIT), interview-defined hostility was found to be a significant predictor of CHD after adjustment for other risk factors (Dembroski, MacDougall, Costa, \& Grandits, 1989; Hecker et al., 1988; Matthews, Glass, Rosenman, \& Bortner, 1977). This association was most strong in younger subjects (below 48 years of age).

Several prospective studies on the association between hostility (as measured by the Cook-Medley Hostility Scale) and CHD have been published. Cook-Medley scores were found to be associated with both CHD incidence and all-cause mortality (Barefoot, Dahlstrom, \& Williams, 1983; Barefoot, Dodge, Peterson, Dahlstrom, \& Williams, 1989; Shekelle, Gale, Ostfeld, \& Paul, 1983). These positive results contrast with three studies that failed to demonstrate a prospective association between CookMedley scores and CHD incidence (Hearn, Murray, \& Luepker, 1989; Leon, Finn, Murray, \& Bailey, 1988; McCranie, Watkins, Brandsma, \& Sisson, 1986).

Other prospective evidence of the possible role of hostility in the incidence of CHD comes from a Finnish study. In this study, a three-item scale measuring hostility, was strongly associated with an increased risk of ischemic heart disease (IHD) (Koskenvuo et al., 1988).

In conclusion, the results of most cross-sectional and prospective studies to date support the hypothesis that people with hostile tendencies are at an increased risk of future CHD. However, the vast majority of studies on the possible role of hostility in the etiology of CHD have been carried out in the United States. Research of the association between hostility - especially as measured by the Cook Medley Hostility Scale - and CHD in European countries is lacking. Therefore, the main purpose of the 
present case-control study was to test the association between hostility and clinically manifest CHD in adult male patients in a Dutch sample. It was hypothesized that: (a) myocardial infarction (MI) cases would report higher levels of hostility than controls, and that (b) the association between hostility and MI would be stronger for younger men than for older men.

\subsection{METHOD}

\section{Subjects and procedure}

The subjects in this case-control study consisted of 249 men between 35 and 65 years of age from the South Limburg region of the Netherlands. The cases were 81 patients hospitalized for a first documented MI. They were admitted to two regional hospitals during the period of January 1990 - December 1990 and constituted a consecutive series. The definite diagnosis of first MI was based on the clinical history taken by a cardiologist, standard ECG readings, and plasma enzyme elevations. Patients with another major illness (e.g. cancer) were excluded from the study.

Once a week the cardiological departments of both hospitals were visited by the first author. All patients with a first MI were invited to participate in the study after they had been clischarged from the coronary care unit. After the purpose and the procedures of the study had been explained, a written informed consent form was signed and a set of questionnaires left behind. Besides the Cook and Medley Hostility Scale, this set consisted of the Buss-Durkee Hostility Inventory (BDHI) (Buss \& Durkee, 1957), the Maastricht Questionnaire $(M Q)$, a 21 -item checklist to assess mental precursors of myocardial infarction (Appels, Höppener, \& Mulder, 1987), and the London School of Hygiene Cardiovascular Questionnaire for the assessment of angina pectoris within the last six months (prior to MI) (Rose, Blackburn, Gillum, \& Prineas, 1982). Also included were variables such as marital status, educational level, smoking. coffee consumption, high blood pressure, diabetes mellitus, and family history of CHD. Patients were requested to complete these questionnaires as soon as possible and to return them not later than two weeks after discharge from the hospital. Finally, a tentative appointment was made for an interview at home about four weeks after discharge from the hospital. During this home visit the Type A behavior pattern (TABP) was assessed by means of the Structured Interview (SI) (Rosenman, 1978); the Maastricht Interview for Vital Exhaustion (MIVE) was employed to measure mental precursors of MI (Meesters \& Appels, 1995a). In all, 94 MI cases were invited to participate, 13 of whom refused. The final response rate was $86 \%$.

The control group consisted of 168 age-matched neighborhood controls (NCs) who were living on the same or adjacent street as cases at the time of the latter's myocardial infarction. The selection of persons who reside in the same neighborhood as the patients yields a reference group representing the source population (Miettinen, 
1985. People with previously documented MI or those having a major illness (e.g. cancer) at the time were excluded from the study. NCs were randomly selected from the telephone directory and were subsequently mailed the same set of questionnaires between January 1990 and May 1991. On receipt of the questionnaires an appointment was made for an interview at home. The interview, which was identical to the interview with the cases, took place roughly four weeks after mailing the questionnaires. In all, 204 eligible controls were contacted, 168 of whom eventually agreed to participate in the present study.

\section{Instruments}

Both cases and controls completed the Dutch version of the Cook and Medley Hostility Scale. This scale was empirically derived from the MMPI and was originally constructed to determine teacher aptitudes for classroom teaching (Cook \& Medley, 1954). It is a paper-and-pencil test consisting of 50 true-false items. Cook-Medley scores range from 0 to 50 . The scale shows high levels of internal consistency, with an average Cronbach's $\alpha$ of about 0.80 (Smith \& Frohm, 1985). Empirical evidence with respect to the stability of Cook-Medley scores is mixed. Two studies - with a 4-year gap in testing - report test-retest reliability coefficients greater than 0.80 (Barefoot et al., 1983; Shekelle et al., 1983), whereas in a more recent study Cook-Medley scores proved to be substantially less stable over a 22-year period, with a reliability coefficient of 0.39 (Siegler et al., 1990).

Although a couple of studies (Barefoot et al., 1989; Costa, Zonderman, McCrae, $\&$ Williams, 1986) suggest the presence of several factors, there is a general consensus that the Cook-Medley Hostility Scale largely measures cynical hostility (Smith \& Pope, 1990). In general, people with high scores on this scale are described as having a cynical, mistrustful attitude toward others.

To date, little is known about the psychometric characteristics of this questionnaire with respect to the Dutch population. Therefore, we have looked at the reliability and validity of the scale in more detail. The Cook-Medley Hostility Scale has good internal consistency with a Cronbach's $\alpha$ of 0.85 in the present sample. Principal component factor analysis reveals the presence of only one factor, which suggests that the CookMedley Hostility Scale is a general index of cynical mistrust (Williams \& Barefoot, 1988).

\section{Data analysis}

Univariate differences between the case group and the control group were tested by means of t-tests. Subsequently, univariate $t$-tests were used to test possible differences on the Cook-Medley Hostility Scale for three different age strata: 35-49 years, 50-59 years, and 60-65 years. Multiple logistic regressions were computed in order to examine differences between cases and controls after adjusting for several control 
variables using the SPSS/PC 4.0 (Norusis, 1990). Patient status (MI or NC) was employed as the dependent wariable, the controls serving as the referent group.

Control variables in the multivariate analyses consisted of age, current smoking, and high blood pressure. Age was included as a continuous variable, high blood pressure as a dichotomous variable based on self-report, and current smoking, defined as a positive answer to the question "Did you smoke during the last six months prior to MI or filling out the questionnaire?", as a dichotomous variable. Moreover, an interaction between hostility and age was included.

\section{$2.4 \quad$ RESULTS}

Characteristics of cases and controls with regard to age, current smoking, and high blood pressure are presented in table 2.1. Cases and controls were well matched for age, their combined mean age being about 55 years. Current smoking occurred significantly more often in cases. Fifty-five cases $(68 \%)$ reported that they had been smoking during the last half year prior to their MIs, as did 66 controls (39\%). There proved to be no significant difference between cases and controls in the prevalence of self-reported high blood pressure.

Table 2.1. Characteristics of cases and controls with respect to age, current smoking, and high blood pressure

\begin{tabular}{|c|c|c|c|c|}
\hline $\mathbf{N}$ & $\begin{array}{l}\text { MI } \\
(\mathbf{8 1})\end{array}$ & $\begin{array}{l}\mathrm{NC} \\
(168)\end{array}$ & & \\
\hline Age & $\begin{array}{l}55.6 \\
(\mathrm{sd}=6.8)\end{array}$ & $\begin{array}{l}54.9 \\
(\mathrm{sd}=6.9)\end{array}$ & $\mathrm{t}=0.71$ & $p=0.48$ \\
\hline Current smoking & $55(68 \%)$ & $66(39 \%)$ & $x^{2}=17.9$ & $p=0.00$ \\
\hline High blood pressure & $17(21 \%)$ & $32(19 \%)$ & $x^{2}=0.13$ & $p=0.72$ \\
\hline
\end{tabular}

The results of the univariate analyses of the Cook-Medley Hostility Scale are displayed in table 2.2. The MI case group had slightly higher scores than the control group ( 22.1 vs $20.7 ; p=0.18$ ). However, this difference is statistically not significant. As mentioned above, several studies have reported a possible age dependence of the association between hostility and MI. Hostility was found to be a stronger predictor of CHD at younger ages (Siegman et al., 1987; Williams et al., 1988; Williams, Barefoot, \& Shekelle, 1985). To examine this possible association, the sample was divided into three different age strata. In order to get a reasonable number of cases and controls in each of the three strata, we created one group of persons younger than 50 years, another group between 50 and 59 years of age, and a third group older than 60 . As can be seen in table 2.2 , only cases in the youngest age group had significantly higher Cook-Medley scores $(24.5$ vs $20.1 ; \mathrm{p}<0.05)$ than controls. Above the age of 
50 there was hardly any difference in mean Cook-Medley scores between cases and controls. These univariate results lend support to the hypothesis that hostility might be a risk indicator, especially for younger people.

Table 2.2. Results of the univariate anallyses of the Cook-Medley Hostility Scale for the total sample and for three different age groups comparing cases and controls by means of t-test

\begin{tabular}{|c|c|c|c|c|c|c|}
\hline & & $\mathbf{N}$ & Mean & s.d. & t & p(two-tailed $)$ \\
\hline \multirow[b]{2}{*}{ Total sample } & $\mathrm{MI}$ & 81 & 22.1 & 8.0 & \multirow[b]{2}{*}{1.34} & \multirow[b]{2}{*}{0.18} \\
\hline & & 167 & 20.7 & 7.5 & & \\
\hline \multicolumn{7}{|l|}{ Age } \\
\hline \multirow[b]{2}{*}{$<50$ years } & MI & 20 & 24.5 & 8.7 & \multirow{2}{*}{2.03} & \multirow{2}{*}{0.04} \\
\hline & $\mathrm{NC}$ & 41 & 20.1 & 7.6 & & \\
\hline \multirow{3}{*}{$50-59$ years } & MI & 34 & 21.6 & 7.8 & \multirow{3}{*}{0.40} & \multirow{3}{*}{0.70} \\
\hline & & & & & & \\
\hline & $\mathrm{NC}$ & 78 & 21.0 & 6.9 & & \\
\hline \multirow[b]{2}{*}{$\geq 60$ years } & MI & 27 & 21.0 & 7.8 & \multirow{2}{*}{0.12} & \multirow[b]{2}{*}{0.90} \\
\hline & $\mathrm{NC}$ & 48 & 20.8 & 8.5 & & \\
\hline
\end{tabular}

Logistic regression analysis, while taking into account the effects of current smoking and high blood pressure, clearly suggested the presence of an interaction between Cook-Medley scores and age $(\beta=-0.005$, s.e. $=0.003 ; \mathrm{p}=0.06)$. These results indicate that in this sample hostility, as measured by the Cook-Medley Hostility Scale, is associated with MI only in interaction with age. In order to provide better insight into the nature of the association between Cook-Medley scores and MI within the three age groups, a logistic regression was computed with age as a categorical variable, again simultaneously controlling for current smoking and high blood pressure. The results may give an indication of the strength of the association between Cook-Medley scores and MI for the three age groups. It should be noted that the predictor variable hostility is represented by a continuous scale with scores ranging from 0 to 50 , which means that the odds ratios refer to one-unit incremental increases in risk. In general, the final logistic regression produced a pattern of results similar to that of the univariate findings (see table 2.2). Only for 35 to 49 -year-old men did the association between Cook-Medley scores and MI prove to be statistically significant $(O R=1.09, p=0.04)$, whereas for males of the two older age groups ( $50-59$ years and $\geq 60$ years) CookMedley scores were not related to $\mathrm{MI}(\mathrm{OR}=1.00, \mathrm{p}=0.90$ and $\mathrm{OR}=1.00, \mathrm{p}=0.94$ respectively). 


\subsection{DISCUSSION}

The possible association between hostility and CHD has been amply studied since the late seventies. However, most studies have been executed in the United States. Hence the main aim of the present case-control study was to test the association between hostility and CHD in a Dutch sample. The results only partially supported the hypotheses of the study. MI patients did describe themselves as exhibiting somewhat higher levels of hostility than controls. However, the difference between the mean scores of cases and controls on the Cook-Medley Hostility Scale was not statistically significant. This somewhat higher score was mainly related to the number of younger males, for whom hostility proved to be a statistically significant risk indicator for MI. The results of the multivariate analyses clearly indicated the existence of an interaction between hostility and age. Hostility only seems to confer a risk for people who are younger than 50. The present findings are in line with the outcome of some previous studies. In a study by Siegman et al. (1987) it was shown that the expressive aspects of hostility, as measured by the Buss Durkee Hostility Inventory, were significantly associated with angiographically documented severity of coronary artery disease (CAD) for patients 60 years of age and younger only. In another study, Dembroski and his co-workers re-analyzed structured interview (SI) ratings of the MRFIT study. Only total potential for hostility and the stylistic hostility sub-component were significantly associated with CHD incidence. These associations were independent of traditional risk factors and appeared only in subjects 47 years of age or younger (Dembroski et al., 1989).

In sum, several cross-sectional as well as prospective studies provide evidence for an age-dependent relationship between hostility (both SI-defined hostility and hostility as measured by self-report questionnaires) and cardiovascular disease. These findings are consistent with those reported in Type $\mathrm{A}$ behavior pattern studies (Williams et $\mathrm{al}$., 1988). The results of the present study are also in accord with the MRFIT data, which displayed a decreasing strength of prediction of the traditional risk factors with adwancing age (Kannel, Neaton, \& Wentworth, 1986). This diminishing of effect might be attributable to survival elfects (Willians et al., 1988).

A few notes of caution apply while interpreting the findings of the present study. First, the most important limitation is undoubtedly its retrospective nature. Inherent to retrospective designs is the possibility of recall bias, or "search for meaning", which happens when cases are likely to overestimate their own risk factor levels in order to attribute their disease to some presumed cause. The present study used a neighborhood control group, something which has advantages as well as disadvantages (Ryu, Thompson, \& Crouse, 1989; Wacholder, Siverman, McLaughlin, \& Mandel, 1992). Sackett (1979) argues that studies using hospitalized controls are less susceptible to recall bias than studies using healthy community controls. On the other hand, the advantages of using neighborhood controls include the greater likelihood that they will be free of serious disease, something which may be associated with hostility as well, and the 
greater likelihood of socioeconomic similarity to cases (Vernick, Vernick, \& Kuller, 1984).

Second, selection bias might have affected the results of this study. Since no significant differences could be detected between participating and non-participating cases with respect to age, current smoking, high blood pressure, or history of angina pectoris, it seems unlikely that non-participating cases caused any selection bias. With regard to neighborhood controls, some selection bias may have affected the results of the study to some extent. During the period of participation, it was occasionally observed that older subjects and/or those who were working seemed to be somewhat less willing to participate in the present study than subjects who were not working because of health problems, unemployment, etc.

Third, the significant association between Cook-Medley scores and MI in the younger age group may be explained by a stronger emotional reaction of these MIcases to the cardiac event. Younger males may react with more anger, resentment, and irritability after a MI, because these patients probably perceive a MI as having more impact on their future life and career outlook. On the other hand however, the studies by Dembroski et al. (1989) and Williams et al. (1988), both prospective in nature, showed that the association between hostility and CHD was mainly observed in younger participants. The results of these follow-up studies indicate that it is not very likely that the observed differences in the young age group in the present study have been inffuenced by a stronger emotional reaction of younger cases after MI.

In conclusion, the results of this case-control study suggest that hostility, as measured by the 50-item Cook-Medley Hostility Scale is associated with an increased risk of first myocardial infarction in adult males. However, the present findings indicate that this association is confined to people younger than 50 years of age since no association was observed in older subjects. 


\section{CHAPTER 3}

\section{DIMENSIONS OF HOSTILITY AND MYOCARDIAL INFARCTION IN ADULT MALES ${ }^{1}$}

\subsection{SUMMARY}

The present case-control study investigated the association between dimensions of hostility and myocardial infarction (MI) in adult males. Hostility was measured with the Buss-Durkee Hostility Inventory (BDHI), which assesses two distinct dimensions of hostility, namely experiential and expressive hostility. Cases were 81 males who were admitted in hospital because of a first MI. The reference group consisted of 168 age-matched, healthy male neighborhood controls. Analyses revealed that especially experiential hostility (comprised of the subscales Resentment and Suspicion) was significantly associated with MI. These results are in contrast with the findings of previous studies, which showed expressive hostility to be positively related to coronary heart disease (CHD). Explanations for these contradictory findings are discussed.

\subsection{INTRODUCTION}

At the end of the seventies the focus of research on psychosocial risk factors of coronary heart disease (CHD) has shifted away from the global, broadly defined Type A behavior pattern (TABP), characterized by extremes of competitiveness, achievement striving, time-urgency, and aggressiveness, to identifying the separate, specific components within this behavior pattern. More specifically, recent studies have directed their attention to hostility supposedly being the toxic element of TABP (for a thorough review see Smith, 1992).

Evidence for the association between hostility and CHD has been provided both by prospective and cross-sectional studies (Barefoot, Dahlstrom, \& Williams, 1983; Barefoot, Dodge, Peterson, Dahlstrom, \& Williams, 1989; Haynes. Feinleib, \& Kannel, 1980; Shekelle, Gale, Ostfeld, \& Paul, 1983; Siegman, Dembroski, \& Ringel, 1987; Williams et al., 1980). To measure hostility, these studies used structured interview (SI) (Rosenman, 1978) ratings (Dembroski, MacDougall, Costa, \& Gran dits, 1989; Dembroski, MacDougall, Williams, Haney, \& Blumenthal, 1985; Hecker,

'Meesters, C.M.G., Muris, P., \& Backus, I.P.G. (1995). Dimensions of hostility and myocardial infarction in adult males. Journal of Psychosomatic Research, in press. 
Chesney, Black, \& Frautschi, 1988; MacDougall, Dembroski, Dimsdale, \& Hackett, 1985; Matthews, Glass, Rosenman, \& Bortner, 1977) and self-report "paper-andpencil" tests such as the Cook-Medley (Cook \& Medley, 1954) Hostility Scale and the Buss-Durkee (Buss \& Durkee, 1957) Hostility Inventory (BDHI). Although some studies failed to replicate the association between hostility and CHD (Hearm, Murray, \& Luepker, 1989; Helmer, Ragland, \& Syme, 1991; Leon, Finn, Murray, \& Bailey, 1988; MoCranie, Watkins, Brandsma, \& Sisson, 1986), the majority support the hypothesis that hostility contributes to an increased risk of CHD. For example, a recent study by Meesters and Smulders (1994) showed that hostility as measured by the Cook-Medley Hostility Scale was significantly associated with MI in men.

However, it should be noted that several studies have clearly demonstrated that hostility is a multidimensional construct, and that probably not all of its aspects are associated with CHD. The results of a number of factor-analytic studies, which have used the Buss-Durkee Hostility Inventory (BDHI) in particular, repeatedly indicated the presence of two distinct factors (Bendig, 1962* Bushman, Cooper, \& Lemke, 1991; Buss \& Durkee, 1957; Musante, MacDougall, Dembroski, \& Costa, 1989; Sarason, 1961). The first factor is represented by items measuring the experience of anger and hostility. This so-called experiential factor is also known as neurotic hostility, because of its strong relationship with anxiety and neuroticism (Costa, McCrae, \& Dembroski, 1989; Siegman, Anderson, Herbst, Boyle, \& Wilkinson, 1992; Siegman et al., 1987). The second factor, which contains primarily items assessing the outward expression of anger and hostility, is labeled expressive hostility and appears to be unrelated to measures of anxiety (Dembroski \& Costa, 1987). Siegman and his co-workers conducted an important study on angiography patients, which showed that the expressive hostility factor of the BDHI was positively associated with coronary artery disease (CAD), whereas experiential hostility was found to be negatively related to severity of CAD (Siegman et al., 1987). These associations were restricted to patients below the age of 60 , whereas the findings were not statistically significant in older patients. Note, in passing, that the latter result is in line with the finding of several other studies that the predictive or discriminating power of risk factors of CHD appears to be most strong among younger persons [e.g. Kannel, Neaton, \& Wentworth, 1986; Williams et al., 1988).

So far, no epidemiological European study on the association between the several dimensions of hostility as indexed by the $\mathrm{BDHI}$ and $\mathrm{CHD}$, has been reported of. Because the subjects of the case-control study by Meesters and Smulders (1994) also completed the BDHI, it became possible to investigate this issue in this particular sample. More specifically, the present paper sought to address the following questions: a) is there an association between the dimensions of hostility as indexed by the BDHI and clinically documented CHD in adult males from the Netherlands? and b) is the association between hostility and CHD age-dependent? 


\subsection{METHOD}

\section{Subjects and procedure}

Two-hundred forty-nine men between 35 and 65 years of age participated in this study. The cases were 81 consecutive patients hospitalized for a first documented MI in the two largest hospitals in the South Limburg region of the Netherlands. The diagnosis of definite first MI was based on a) the clinical history taken by a cardiologist; b) standard ECG readings; and c) maximum plasma enzyme levels. In all, 94 MI patients were contacted, 13 of whom refused to participate in the study, yielding a response rate of $86 \%$.

Control subjects were 168 age-matched neighborhood controls (NCs) who were residing in the same or adjacent street as the $\mathrm{MI}$ patients at the time of the latter's hospitalization. They were selected at random from local telephone directories. In this way, a reference group representative for the source population was created (Miettinen, 1985). Controls were included only if they had no history of MI (selfreport). Altogether, 204 eligible controls were invited to participate in the study, 168 of whom finally did so.

Both cases and controls completed a set of questionnaires and were subsequently interviewed at home. A more extensive description of the procedure and instruments is presented elsewhere (Meesters \& Smulders, 1994).

\section{Instruments}

Hostility was assessed by means of the Buss Durkee Hostility Inventory (BDHI) (Buss \& Durkee, 1957), a 75 true-false item self-report questionnaire. The scale supplies information on the individual's level of general hostility and on seven aspects of hostile behavior: Assault, Indirect Hostility, Verbal Hostility, Irritability, Negativism, Resentment, and Suspicion. As mentioned in the introduction, evidence from several studies thas suggested the presence of two distinct factors, an experiential factor, primarily represented by the Resentment and Suspicion subscales, and an expressive factor, primarily defined by the Assault, Verbal Hostility, Indirect Hostility, and Irritability subscales of the BDHI (e.g. Bendig, 1962; Bushman et al., 1991; Buss \& Durkee, 1957; Musante et al., 1989). Two week test-retest reliability coefficients have been reported to range from 0.64 to 0.78 for the subscales, and to be 0.82 for the total score (Biaggio, Supplee, \& Curtis, 1981). The literature has provided various validity relationships of the BDHI with several other self-report measures of anger, hostility, and aggression ranging from 0.4 to 0.7 (Matthews, Jamison, \& Cottington, 1985).

In the present study, a Dutch translation of the BDHI was employed using a trueT-false response format. Factor analysis (principal components with VARIMAX rotation) of the Dutch BDHI suggests the existence of two factors which resemble very closely to the expressive and experiential factors, as found by Buss and Durkee 
in their study with the original BDHI (Buss \& Durkee, 1957). Furthermore, this instrument has good internal consistency with Cronbach's $\alpha$ 's of $0.88,0.76$, and 0.85 for BDHI total score, the expressive factor and the experiential factor, respectively. Test-retest reliability with a two-year gap was $I=0.78$, indicating that Dutch $B D H I$ scores are reasonable stable ower time (Neissen, 1993).

\section{Data analysis}

Univariate data analysis consisted of t-tests comparing the MI case group and the control group with respect to BDHI total score, each of the BDHI subscales, and the expressive and experiential factor separately.

The associations between several dimensions/aspects of hostility and $\mathrm{MI}$ were subsequently evaluated by means of multiple logistic regression analyses, in which patient status (MI or NC) was the dependent variable, the controls being the referent group. Control variables in the logistic regression equations consisted of age, current smoking and high blood pressure. Age was included as a continuous variable, current smoking and high blood pressure as dichotomous variables. Current smoking and high blood pressure were defined as positive answers to the questions "Did you smoke during the last six months prior to MI or completing the questionnaire?", and "Did you receive treatment for high blood pressure during the six months preceding hospitalization (cases) or filling out the questionnaire (controls)? " In addition, interactions between the distinct aspects of hostility and age were calculated. The data were analysed using SPSS/PC 4.0 (Norusis, 1990).

\section{$3.4 \quad$ RESULTS}

Some health and demographic characteristics of cases and controls are presented in table 3.1. Both MI cases and controls were, on the average, about 55 years old. Current smoking was significantly more prevalent among cases than controls. There was no difference between cases and controls with respect to the prevalence of selfreported high blood pressure.

Table 3.2 displays the results of the univariate analyses of the several BDHI scales. First of all, no significant difference between cases and controls was found with respect to level of general hostility, as indexed by BDHI total scores, means being $50.9(\mathrm{SD}=18.4)$ and $48.5(\mathrm{SD}=17.1)$, respectively $(\mathrm{t}=0.99 ; \mathrm{p}=0.34)$. Second, when we take a look at the different aspects of hostility, a different picture emerges. As shown in table 3.2, MI patients had significantly higher mean Resentment $(3.7$ vs. $2.9 ; p=0.04)$ and Suspicion $(6.5$ vs. $5.4 ; p=0.04)$ scores than control subjects. Mean scores on the other BDHI subscales were rather similar for the two groups. Finally, the experiential factor discriminated significantly between cases and controls, means being $10.3(\mathrm{SD}=5.8)$ and $8.4(\mathrm{SD}=5.9)$, respectively $(\mathrm{t}=2.34$; $\mathrm{p}=0.02)$. 
Table 3.1. Demographic and health characteristics of the sample

\begin{tabular}{lllll}
\hline $\mathbf{N}$ & $\begin{array}{l}\text { MI } \\
(\mathbf{8 1})\end{array}$ & $\begin{array}{l}\text { NC } \\
(\mathbf{1 6 8})\end{array}$ & & \\
\hline Age & 55.6 & 54.9 & $\mathrm{t}=0.71$ & $\mathrm{p}=0.48$ \\
& $(\mathrm{sd}=6.8)$ & $(\mathrm{sd}=6.9)$ & & \\
Current smoking & $55(68 \%)$ & $66(39 \%)$ & $x^{2}=17.9$ & $\mathrm{p}=0.00$ \\
High blood pressure & $17(21 \%)$ & $32(19 \%)$ & $x^{2}=0.13$ & $\mathrm{p}=0.72$ \\
\hline
\end{tabular}

Table 3.2. Mean scores and standard deviations on BDHI total, 7 BDHI subscales, BDHI expressive factor, and BDHI experiential factor comparing cases $(n=81)$ and controls $(n=168)$ by means of t-tests

\begin{tabular}{|c|c|c|c|c|c|c|}
\hline \multirow[b]{2}{*}{ Scale } & \multicolumn{2}{|c|}{ MI } & \multicolumn{2}{|c|}{$\mathrm{NC}$} & \multirow[b]{2}{*}{$\mathrm{t}$} & \multirow[b]{2}{*}{$p(t w s-$ lalled $)$} \\
\hline & Mean & sdl & Mean & sd & & \\
\hline BDHI total & 50.9 & 18.4 & 48.5 & 17.1 & 0.99 & 0.34 \\
\hline Assault & 7.1 & 3.8 & 6.3 & 3.9 & 1.59 & 0.12 \\
\hline Indirect Hostility & 6.7 & 3.7 & 6.7 & 3.7 & -0.02 & 0.98 \\
\hline Inritability & 8.8 & 4.9 & 8.8 & 4.3 & 0,04 & 0.98 \\
\hline Negativism & 4.9 & 2.4 & 4.4 & 2.3 & 1.42 & 0.16 \\
\hline Resentment & 3.7 & 2.9 & 2.9 & 2.8 & 1.98 & 0.04 \\
\hline Suspicion & 6.5 & 3.7 & 5.4 & 3.8 & 2.13 & 0.04 \\
\hline Verbal Hostility & 13.2 & 5.1 & 13.8 & 5.11 & -0.90 & 0.38 \\
\hline Expressive factor & 35.8 & 14.0 & 35.5 & 12.7 & 0.14 & 0.89 \\
\hline Experiential factor & 10.3 & 5.8 & 8.4 & 5.9 & 2.34 & 0.02 \\
\hline
\end{tabular}

As mentioned earlier, an age-dependent relationship between hostility and CHD has been reported in the epidemiological literature more than once le.g. Meesters \& Smulders, 1994; Siegman et al., 1987; Williams et al., 1988; Williams, Barefoot, \& Shekelle, 1985). Therefore, to examine the possible interaction between hostility and age, a series of multiple logistic regression analyses (for each BDHI subscale separately), including an interaction term, were performed. None of the interactions proved to be statistically significant, with p-values ranging from 0.14 to 0.90 .

Subsequently, the association between the several BDHI measures and MI was investigated by means of separate multiple logistic regression analyses. The results of these analyses are displayed in table 3.3. The logistic regression models, while adjusting for age, current smoking, and high blood pressure for each of the hostility measures separately, yielded a pattern of results very similar to that of the univariate outcomes. The BDHI experiential factor $(\beta=0.052, \mathrm{p}=0.03)$ was found to be statistically significant associated with MI. The Resentment $(\beta=0.094, p=0.06)$ and Suspicion $(\beta=0.071, \mathrm{p}=0.05)$ subscales, which discriminated significantly between cases 
and controls univariately, approached statistical significance in the multivariate analyses. No further significant findings emerged.

Table 3.3. Logistic regression coefficients of each of the hostility measures predicting case group status (MI, NC) with the controls being the referent group, controlling for age, current smoking, and high blood pressure

\begin{tabular}{llll}
\hline Scale & $\beta$ & se & p(two-tailed) \\
\hline BDHI total & 0.008 & 0.008 & 0.36 \\
& & & \\
Assatlit & 0.047 & 0.036 & 0.19 \\
Indirect Hostility & 0.004 & 0.040 & 0.93 \\
Irritability & 0.004 & 0.032 & 0.90 \\
Negativism & 0.058 & 0.060 & 0.34 \\
Resentment & 0.094 & 0.051 & 0.06 \\
Suspicion & 0.071 & 0.037 & 0.05 \\
Verbal Hostility & -0.030 & 0.029 & 0.30 \\
& & & 0.95 \\
Expressive factor & 0.001 & 0.011 & 0.03 \\
Experiential factor & 0.052 & 0.024 & \\
\hline
\end{tabular}

\subsection{DISCUSSION}

The main purpose of the present case-control study was to examine the relationship between hostility and its several components as measured by the BDHI on the one hand and CHD on the other hand. Furthermore, since an age-dependent association between hostility and CHD has been reported repeatedly in the literature, the interaction between age and hostility was investigated. In the current study, individual's level of general hostility (i.e. BDHI total score) was not found to be significantly associated with MI. Note that this finding is in contrast with the results of the previous study of Meesters and Smulders (1994). In that study, employing the same sample of subjects, general hostility, as indexed by the Cook-Medley Hostility Scale, was found to be significantly associated with MI. These diverging findings may be due to the fact that the Cook-Medley Hostility Scale and the BDHI assess different aspects of the hostility construct. The fact that BDHI and Cook-Medley scores are only moderately correlated (Smith \& Frohm, 1985) ${ }^{2}$ supports this idea. Besides, as mentioned in the introduction, several other authors have suggested that probably not all aspects of hostility are associated with an elevated risk for CHD (Siegman et al.,

${ }^{2}$ In the present sample, product-moment correlations between Cook-Medley scores and the several BDHI scores were as follows: Assault $(r=0.35)$, Indirect Hostility $(r=0.36)$, Irritability $(r=0.47)$. Negativism $(r=0.40)$, Resentment $(r=0.54)$, Suspicion $(r=0.64)$, Verbal Hostility $(r=0.29)$. Experientiall factor $(r=0.68)$, and Expressive factor $(r=0.51)$. 
1992; Siegman et al., 1987). The results of the univariate analyses of the separate BDHI subscales and factors confirm this notion. Compared to control subjects, MI cases only exhibited higher scores on the BDHI subscales of Resentment and Suspicion. In line with this, cases showed higher scores on the BDHI experiential factor (which is composed of the Resentment and Suspicion subscales).

No significant interaction was observed between hostility, as indexed by the $\mathrm{BDHI}$, and age. In the multivariate analyses, when controlling for age, current smoking, and high blood pressure, the associations becween hostility and CHD were less pronounced, i.e. only the association between the BDHI experiential factor on the one hand and MI on the other hand remained statistically significant. The finding that the association between hostility and CHD became less pronounced when testing multivariately is probably due to the fact that hostility is positively associated with smoking (Dembroski et al., 1989). Consequently, hostility may confer risk for CHD not only by virtue of psychophysiological mechanisms but also because highly hostile subjects are inclined to smoke more.

It is important to note that the main result of the present study, namely the finding that the experiential factor of the BDHI was significantly associated with $\mathrm{MI}$, is in contradiction with the results of other studies on the relationship between dimensions of hostility and CHD. Evidence so far has indicated that, in particular, the expressive factor of the BDHI is positively associated with CAD (for an overview see Siegman, 1994b). The aberrant result of the present study can be explained in several ways. First of all, most of the studies using the BDHI have investigated cardiovascular reactivity with healthy and rather young subjects who are free of CAD (Suls \& Wan, 1993). Up to now, there is only one clinical study that has investigated the association between differential aspects of hostility, as measured by the BDHI, and CAD (Siegman et al., 1987). In that study, Siegman and his co-workers found that the expressive factor of the BDHI was positively associated with the severity of CAD, whereas the experiential factor was even negatively related to cardiovascular pathology. Note that they also found that the personality dimension of neuroticism was significantly associated with experiential hostility. The latter finding may be important, since Siegman and colleagues (1987) studied a sample of angiography patients. It is known that neurotic people are more prone to report cardiovascular-like complaints (such as chest pain) in the absence of any evidence of coronary heart disease (Costa, Zonderman, McCrae, \& Williams, 1986). Hence, it is not unconceivable that patients with high levels of neuroticism but free from disease are more likely to be referred for diagnostic cardiac catheterization. In other words, it may well be the case that in the angiography sample of Siegman et al. (1987) neurotic, but healthy, subjects were overrepresented. This may have caused a "spurious" inverse association between experiential hostility and CAD. Obviously, there is a need for additional clinical research on the potential risk status of differential BDHI dimensions of hostility.

Second, the difference in results may be attributable to cross-cultural differences. For instance, although previous studies have found evidence for cross-cultural validi- 
ty of the Type A behavior pattern (Hayano et al. 1989; Sykes, Haertel, Gostautas, \& Evans, 1992; Wrzesniewski, Forgays, \& Bonaiuto, 1990), at the same time considerable variation was found between several populations in levels of different components of Type A (Sykes et al., 1992). The same may be the case with hostility in the present sample of Dutch people. Naturally, the present study does not allow conclusions about possible cross-cultural differences with respect to the risk status of dimensions of hostility. This may be a challenge for furure research.

A third explanation refers to the specific characteristics of the subjects, who participated in the present study. It is important to note that $49 \%$ of the present sample was not working anymore, because of retirement or work-incapacity. The majority of studies on the relationship of psychosocial risk factors to cardiovascular disease - especially if they are prospective in nature - are generally based on samples of males who are usually in the middle of their working career. Therefore, it may be so that the substantial number of non-working men in the present study has affected the perception of their own behavior in some way.

It should be noted that differences in self-reports of dimensions of hostility between MI patients and neighborhood control subjects may be influenced by recall bias, due to the retrospective design of the study. However, there is no clear reason why experiential hostility is more likely affected by recall bias than, for example, expressive hostility, for which no significant differences were observed between MI cases and controls. Second, selection bias may be a possible confounder of the findings of this study. However, participating and non-participating cases were quite similar in age, current smoking, high blood pressure, and history of angina pectoris. With respect to neighborhood control subjects, it was sometimes noted that younger persons and those who were not working when contacted tended to be somewhat more inclined to participate.

In conclusion, the results of the present study provide empirical evidence for an association between experientiall aspects of hostility and myocardial infarction in adult men from the Netherlands. It seems that people characterized by high levels of resentment, suspicion, and mistrust are at an increased risk for developing CHD. The contrast of these findings with several other studies, which have been mainly executed in the United States is very intriguing. In order to gain more insight into the role of specific dimensions of hostibity for CHD, further prospective studies in the Netherlands and other European countries as well as in the United States are certainly called for. 


\section{CHAPTER 4}

\section{HOSTILITY AND PERCEIVED PARENTAL REARING BEHAVIOUR ${ }^{1}$}

\subsection{SUMMARY}

The present study investigated the relationship between perceived parental rearing behaviours and individual differences in hostility in a sample of 187 women and 104 men. It was found that high hostile subjects perceived more rejection and overprotection, and less emotional warmth of their parents than low hostile subjects. Furthermore, it was shown that in both sexes perceived paternal rejection was the strongest predictor of level of hostility.

\subsection{INTRODUCTION}

Since the end of the 1950s, a wealth of studies in health psychology and behavioural medicine have investigated the potential influence of psychosocial factors on physical health. Initially, most attention has been directed to the Type A behaviour pattern (TABP), which is an overt behaviour pattern consisting of feelings of time pressure, impatience, competitiveness, aggression, and easily evoked hostility (Friedman \& Rosenman,, 1974). The results of two large prospective studies indicated that TABP was significantly associated with coronary heart disease (CHD) (Haynes, Feinleib, \& Kannel, 1980; Rosenman et al., 1975). Unfortunately, subsequent studies failed to replicate the association between TABP and CHD (e.g. Shekelle et al., 1985). As a consequence the focus of attention has shifted away from global Type A to specific components of this multidimensional construct. Hostility has been put forward as the toxic component of TABP (Willams, 1987) and has received most scientific attention. Most of the available evidence suggests that hostile persons seem to be at increased risk for subsequent CHD and other life-threatening illnesses (for a review: see Smith, 1992).

If hostility proves to be a threat to health, it would be useful. to develop prevention and intervention programs. In order to reduce risk for $\mathrm{CHD}$ associated with high levels of hostility, it is important to identify high hostile people at the beginning of

\footnotetext{
Meesters, C., Muris, P. \& Esselink, T. (1995). Hostility and perceived parental rearing behaviour. Personality and Individual Differences, 18, 567-570
} 
the disease process as well as to comprehend the early contributions to and pathogenic processes connected to the development of hostility. Although serious CHD is seldomly observed in children, the study of Matthews and Woodall (1988) has shown that the atherosclerotic process already starts in adolescence, and biological risk factors for CHD prove to be relatively stable over time in pediatric subjects. Therefore, the study of the process of development of individuall differences in levells of hostility is justifiable.

So far, only a small] number of studies have investigated the role of parental attitudes and behaviours in the development of hostility. Firstly, Smith, Pope, Sanders, Allred, and $O^{\prime} K e e f f e(1988)$ showed that high hostile students described their families of origin as high in conflict and low in cohesion in comparison to their low hostile counterparts. In a related study by Houston and Vavak (1991), subjects were asked to describe retrospectively their parents" behaviours. It appeared that hostility was related to descriptions of parents as having displayed less genuine acceptance, more hostile control, more interference with subjects' desires, and more punitiveness. Finally, Woodall and Matthews (1989) found that hostile children tend to come from families that their parents describe as low in supportiveness and involvement. Altogether, the results of these studies suggest that family environment contributes to hostility. To be more precise, development of hostility seems to be enhanced in families that are characterized by low levels of positive involvement and high levels of aversive interactions.

The present study further investigates the relationship between family characteristics and individual differences in hostility. More specifically, it is examined whether perceived parental rearing behaviours such as emotional warmth, rejection, and overprotection are associated with levels of hostility. A study on the aetiological antecedents of TABP by Emmelkamp and Karsdorp (1987) revealed that perceived parental rearing practices, especially rejection and lack of emotional warmth, were significantIy related to $T A B P$. Building on these findings the present study has been set up. It is expected that subjects characterized by high levels of hostility perceive less emotional warmth and more rejection in comparison with low hostile persons. No specific hypothesis was formulated with repect to the impact of perceived parental overprotection.

\subsection{METHOD}

\section{Subjects}

Initially, the subjects were 331 students (219 women and $112 \mathrm{men}$ ) in schools of Higher Vocational Education (physiotherapy, ergotherapy, speech therapy, and teacher training college). Their mean age was 21.2 years $(S D=2.1$; range $17-34$ yrs). 
Questionnaire data were only used for statistical analysis, if subjects had completed fully a questionnaire measuring one's recollections concerning one's parents' rearing behaviour (the EMBU; Perris, Jacobsson, Lindström, von Knorring, \& Perris, 1980 ). This resulted in a final sample of 291 subjects (187 women and 104 men). The mean age of this final sample was 21.1 years $(S D=2.2$; range $17-34$ yrs).

\section{Measures}

The subjects were asked to complete the following questionnaires:

The EMBU (Perris et al., 1980; Dutch version by Arrindell, Emmelkamp, Brilman, \& Monsma, 1983) is a questionnaire which is designed to assess one's memories of parental rearing behaviour. Each item is scored on a four-point Likert scale (1 $=$ No, never; $2=$ Yes, but seldom; $3=$ Yes, often; $4=$ Yes, most of the time). Factor analytic research has revealed three primary factors (i.e. scalles) which possess cross-national invariance (Arrindell \& van der Ende, 1984; Arrindell et al., 1986). The first factor, rejection ( 25 items), is characterized by physical punishment, rejection of the subject as an individual, hostility, lack of respect for subject's point of view and needs, and ridiculizing and/or criticizing his or her inadequacies and problems in front of others. The second factor, labeled emotional warmth (18 items), represents a type of upbringing described as: providing warm and loving attention, giving help to the subject without being intrusive, much respect for the subject's standpoint, the subject having faith in his/her parents, and intellectual stimulation. The third factor, defined as overprotection (16 items), appears to reflect a set of parental behaviours indicative of a person's protection, although in an exaggerated way, a comparatively high llevel of intrusiveness, high standards of achievement, imposement of strict rules, and requiring undisputable obedience. It was demonstrated that the retrospective nature of the inventory did not affect the reliability and validity of gathered data due to recall errors or falsified accounts (Arrindell et al., 1983). In the present study, subjects were required to score each item with respect to their father's and their mother's behaviour separately.

The Cook and Medley Hostility (Ho) scale (Cook \& Medley, 1954) is an inventory that has been empirically derived from the Minnesota Multiphasic Personality Inventory (MMPI). The Ho scale consists of 50 true-false items and scores range between 0 (not hostile at all) and 50 (extremely hostile). There is a general consensus that the Ho scale measures cynical hostility, i.e. a cynical, mistrustful attitude towards others (Meesters \& Smulders, 1994; Smith \& Pope, 1990).

\subsection{RESULTS AND DISCUSSION}

As can be seen in table 4.1, all measures (Ho scalle and EMBU scales) produced acceptable Cronbach's $\alpha$ 's. Furthermore, it was found that men and women differed significantly with respect to EMBU emotional warmth scores: male subjects indicated 
that they had experienced less emotional warmth of both the father $[\mathrm{t}(289)=-2.4$, $p<0.05]$ and the mother $[t(289)=-3.0, p<0.01]$ than female subjects. No sex differences were found on the EMBU rejection and overprotection scales. In addition, paired t-tests confirm previous findings of Perris et al. (1980), that respondents have different judgments of the rearing behaviours of their father and their mother, means being 35.5 vs. $36.1[\mathrm{t}(290)=-1.9, \mathrm{p}<0.06]$ for rejection, 52.9 vs. $55.1[\mathrm{t}(290)=$ $-6.9, p<0.001]$ for emotional warmth, and 33.0 vs. $35.6[t(290)=-9.8, p<0.001]$ for overprotection, respectively.

Table 4.ll. Reliability coefricients and mean scores (SDs) on hostility and EMBU measures for men $(n=104)$ and women $(n=187)$ separately

\begin{tabular}{lllll}
\hline & \multicolumn{2}{c}{ Cronbach's $\alpha$} & \multicolumn{2}{c}{ Mean Score (SD) } \\
& Men & Women & Men & Women \\
\hline Ho (hostility) & 0.70 & 0.65 & $20.7(6.3)$ & $18.0(5.9)^{* * * *}$ \\
EMBU & & & & \\
Of fachers & & & & \\
Rejection & 0.77 & 0.86 & $35.7(5.9)$ & $35.3(7.0)$ \\
Emotional warmth & 0.82 & 0.86 & $51.2(7.8)$ & $53.8(8.9)^{*}$ \\
Overprotection & 0.68 & 0.72 & $32.9(5.4)$ & $33.1(6.4)$ \\
Of mothers & & & & \\
Rejection & 0.77 & 0.87 & $35.7(5.8)$ & $36.3(7.2)$ \\
Emotional warmth & 0.81 & 0.84 & $53.4(7.4)$ & $56.1(7.5)^{* *}$ \\
Overprotection & 0.70 & 0.65 & $34.7(5.6)$ & $36.0(6.0)$ \\
\hline
\end{tabular}

Note: Differences between men and women were analysed with t-tests.

$* \mathrm{p}<0.05 ; * * \mathrm{p}<0.01 ; * * * \mathrm{p}<0.001$

In agreement with previous studies on the Ho scale, men obtained significantly higher hostility scores than women [t $(289)=3.6, p<0.001]$ (see e.g. Barefoot et al., 1991; Woodall \& Matthews, 1993). This sex difference in hostility is in line with sex differences in risk for CHD, i.e. CHD is far more common in men than in women (Insull, 1973). This indicates that differences in levels of hostility may yield a partial explanation for the different CHD prevalence among men and women. Because of the observed difference in mean Ho scores, men and women will be treated as separate samples in the further course of this paper.

To analyze the data, both the sample of men and women were divided into a high and a low hostile group according to their score on the Ho scale (by means of a median split procedure). Results of t-test comparisons of high and low hostile men and women on EMBU scales are presented in table 4.2. It appeared that, in both the sample of men and women, high hostile subjects perceived their father and mother as more rejective and overprotective than low hostile subjects. In the male sample, 
however, the relationship between perceived overprotection by the mother and hostillty was only marginally significant. In addition, high hostile men indicated to have experienced lower levels of emotional warmth of both father (but only as a trend) and mother compared to low hostile men. In the sample of women, no significant differences on perceived emotional warmth between high and low hostile subjects emerged.

Table 4.2. Comparison of high hostile and low hostile men and women on EMBU scales

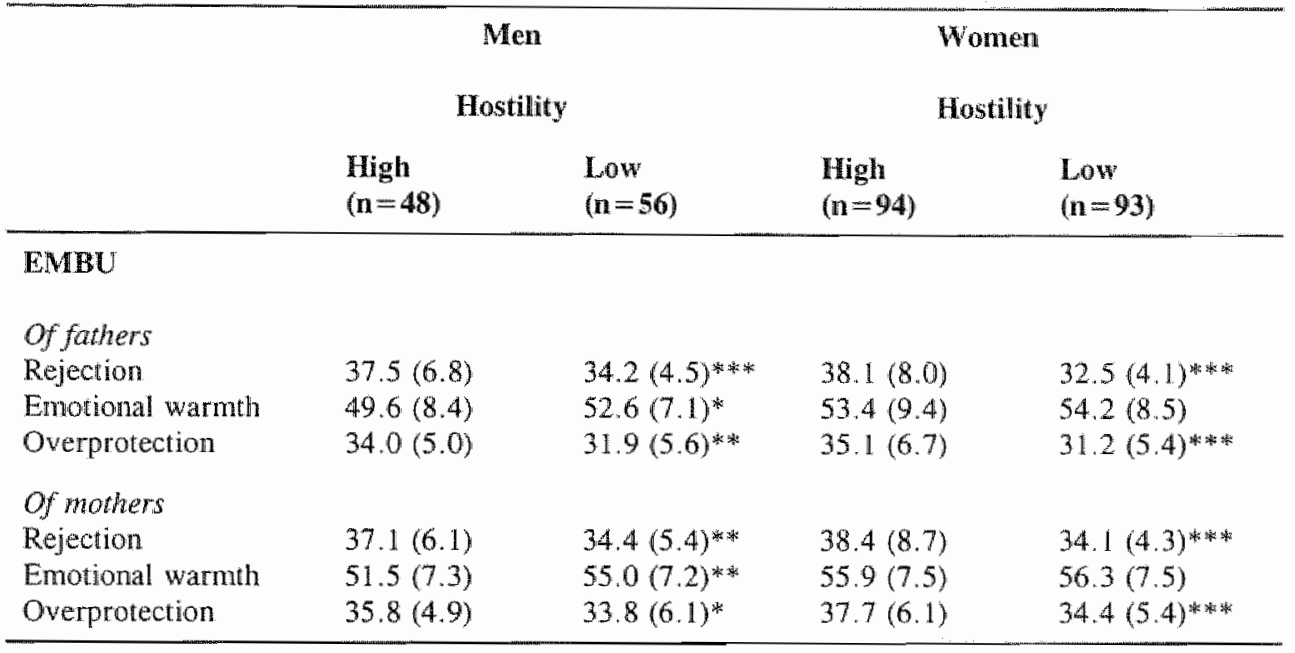

Note: Differences between high and low hostile groups were analysed with t-lests.

${ }^{*} \mathrm{p}<0.10$ *** $\mathrm{p}<0.05$; *** $\mathrm{p}<0.01$

To examine whether the EMBU scales accounted for unique sources of variance in hostility scores, a series of (forward) stepwise regression analyses was carried out. In both the sample of men and women, the regression analyses with perceived rearing behaviours of the father (i.e. rejection, emotional warmth and overprotection) being the predictors and Ho scores being the dependent variable, revealed that only rejection accounted for a significant proportion of the variance [men: $r=0.35$, $F(1,103)=14.3, p<0.001$; women: $r=0.44, F(1,186)=45.7, p<0.001]$.

In men, the regression analysis with perceived rearing behaviours of the mother as predictors and Ho scores being the dependent variable, again, showed that only rejection declared a significant proportion of the variance $[\mathrm{r}=0.33, \mathrm{~F}(1,103)=12.7$, $p<0.001]$. In women, perceived rejection by the mother also entered on the first step $[r=0.33, F(1,186)=22.6, p<0.001]$, whereas overprotection accounted for an extra $2 \%$ of variance [multiple $r=0.36, F(2,185)=14.3, p<0.00 !]$.

The results mentioned above indicate that in both sexes rejection by the father as well as rejection by the mother were related to levels of hostility. To examine the relative strenght of the relationship between maternal and paternal rejection on the one hand and hostility on the other, additional regression analyses were carried out. 
In these analyses rejection by the father and rejection by the mother were included as the predictor variables, whereas hostility was the dependent variable. Results clearly showed that in both sexes paternal rejection was most strongly associated with hostility [men: $r=0.35, F(1,102)=14.3, p<0.001$; women: $r=0.47, F(1,185)=52.7$, $p<0.001]$.

In conclusion, in the present study, a relationship between hostility and perceived parental rearing behaviours was found. More specifically, high hostile subjects perceived more rejection and overprotection, and less emotional warmth of their parents than low hostile subjects. An additional finding was that, in both sexes, rejection, in particular by the father, was most predictive of subject's level of hostility.

The current findings are consistent with previous studies that investigated family characteristics in relation to hostility (Houston \& Vavak, 1991; Smith et al., 1988; Woodall \& Matthews, 1989). The general conclusion of these studies is that negative family characteristics are positively related to hostility. The results of the present study may be interpreted in several ways. One way to interpret the observed relationships is to assume that negative parental rearing behaviours enhance the development of a hostile attitude towards others by means of social learning processes. Second, genetic factors may play a role in the development of hostility in childhood. Genetics may contribute to the aetiology of hostility via two different pathways. One is the inheritance of a tendency for perceiving and reacting to parental (rearing) behaviours, in ways thar are promotive for the development of hostility. Another way is the inheritance of behavioural characteristics that evoke parental behaviours such as described above, which in turn may be conducive to developing hostility. Thus far, results of behavioural genetic research suggests that the observed relations between peceptions of parental behaviours and hostility, both in previous studies and in the present one, may be explained by both genetic variables and socialization influences (see e.g. Plomin \& Bergeman, 1991; Scarr \& McCartney, 1983).

The findings of the present study should be interpreted with caution for several reasons. First, most of the studies (including the present one) can be criticized because of the retrospective nature of the instruments that have been employed to measure parental attitudes and behaviours (e.g. the EMBU). In other words, it may well be the case that hostile subjects, simply because they have a more negative, resentful view of their environment (see Smith, 1992) also perceive the behaviours of their parents as more negative. However, the results of a prospective 4-year follow-up study by Woodall and Matthews (1993) indicate that family characteristics (i.e. support) indeed seem to play a role in the development of hostility. Second, it should be noted that the results of the present study have been obtained in a sample of highly educated, white, young adults. Future research should investigate the above described relationships in other samples. Preliminary results of a related study by Meesters and Muris (1995), employing a sample of middle-aged men of low education, seem to yield a similar pattern of findings as did the present study.

In summary, the findings of the present study support the notion that the development of hostility, defined as a cynical, mistrustfull attitude towards other people, 
Chapter 4

begins in childhood. At the same time, the present results underline the importance of future prospective investigation of the familial antecedents of hostility in children and adolescents in order to gain better comprehension of the determinants of early risk for coronary heart disease. 


\section{CHAPTER 5}

\section{THE RELATIONSHIP BETWEEN HOSTILITY AND PERCEIVED PARENTAL REARING BEHAVIOUR: A STUDY OF MALE MYOCARDIAL INFARCTION PATIENTS AND HEALTHY CONTROLS ${ }^{1}$}

\subsection{SUMMARY}

This study examined the relationship between perceived parental rearing behaviour and individual differences in hostility in a sample of 67 middle-aged male myocardial infarction patients and 139 healthy neighbourhood controls. The EMBU was used to measure perceptions of parental rearing behaviour. Hostility was assessed by means of the Cook-Medley Hostility ( $\mathrm{Ho}_{0}$ scale and the Buss-Durkee Hostility Inventory (BDHI). The present findings are generally in line with the results of our previous study among highly educated young adults (Meesters, Muris, \& Esselink, 1995). The results show that in both cases and controls high hostility is related to perceived parental rejection and perceived lack of emotional warmth. Furthermore, perceived rejection by bath parents seems to be the most strong predictor of individuals' level of hostility. Implications of these findings for future research are discussed.

\subsection{INTRODUCTION}

The association between hostility and coronary heart disease (CHD) has been investigated extensively during the past fifteen years. The study of the psychosocial characteristic hostility as a possible predictor of CHD originated from the initial Type A behaviour pattern (TABP) line of research. Hostility is now supposed to be the central or "toxic" component of the TABP (for a review see Smith, 1992). Although some negative results have been reported (Hearn, Murray, \& Luepker, 1989; Helmer, Ragland, \& Syme, 1991; Leon. Finn, Murray, \& Bailey, 1988; McCranie, Watkins, Brandsma, \& Sisson, 1986), the majority of both cross-sectional and prospective epidemiological studies indicate that hostility involves an elevated risk of CHD (for an extensive review see Helmers, Posluszny, \& Krantz, 1994). People with high levels of hostility are considered to be easily irritated, often angered, prone

'Meesters, C., \& Muris, P. (1995). The relationship between hostility and perceived parental rearing behaviour: A study of male myocardial infarction patients and healthy controls. (submirted) 
to quarrel, suspicious and continuously on their guard against other people. Basically, hostille persons tend to believe that the world is mean and other people are dishonest and selfish. It has been argued that hostility should be considered a multidimensional psychological construct. It has, for instance, been suggested that hostility should be divided into three distinct components (Barefoot, 1992; Barefoot \& Lipkus, 1994): an affective component, including various emotional states, such as anger, annoyance, irritability, resentment, contempt, and disgust; a cognitive component, comprising negative beliefs about other people; and a third component, consisting of a variety of aggressive behaviours, ranging from sulking and verbal aggression to overt physical aggression.

In recent years, research in the field of hostility and health has also paid attention to the childhood origins of hostility. The evaluation of the aetiological antecedents of hostility may be valuable in view of possible prevention and intervention programs. Although children rarely suffer from $C H D$, it has been demonstrated that the process of atherosclerosis starts during a person's teens. Furthermore, biological risk factors of CHD seem to be relatively stable over time, at least in paediatric populations (Matthews \& Woodall, 1988). From this perspective, it would be important to identify highly hostile individuals early in the course of the disease and to understand early contributions to and pathogenic processes involved in the evolution of high levels of hostility.

Up to now, the developmental antecedents of hostility have not been studied extensively. Studies of the developmental aspects of hostility can be divided into heritability studies and studies of possible parental influences. In the last decade, several studies have evaluated the potential genetic basis of hostility (Carmelli, Rosenman, \& Swan, 1988; Matthews, Rosenman, Dembroski, Harris, \& MacDougall, 1984; Mathews, Woodall, Engebretson et al., 1992; Pedersen et al., 1989; Rose, 1988; Smith, McGonigle, Turner, Ford, \& Slattery, 1991). The results of these studies suggest that there is at least a small genetic contribution to the development of hostile attitudes and behaviors.

In the past few years, a limited number of studies of the impact of parental behaviours and attitudes on the development of hostility have been published. In a sample of undergraduate students Smith and his colleagues (1988) found that high scores on the Cook-Medley Hostility (Ho) Scale (Cook \& Medley, 1954) were associated with descriptions of early family environments as less supportive and cohesive, and more conflicual. Similarly, findings of a study by Houston and Vavak (1991) showed that highly hostile undergraduate students rated their parents as more controlling and punitive, and less sincerely accepting. Furthermore, results of a recent investigation of 25 adult male monozygotic (MZ) twin pairs indicated that high Ho scores were associated with high levels of hostile control and low levels of positive involvement from parents (McGonigle, Smith, Benjamin, \& Turner, 1993). In congruence with these retrospectively based correlates of hostility, Woodall and Matthews (1989) showed that hostile children tended to come from famillies with low supportiveness and interpersonal involvement. Summarizing, evidence so far suggests that factors in 
the family environment contribute to children's development of certain psychological characteristics, such as hostility, that may, in later life, place them at risk of CHD.

Recently, we reported on a study of the association between hostility and perceived parental rearing behaviour (Meesters, Muris, \& Esselink, 1995). To be more precise, we investigated the relationship between perceived parental child-rearing behaviour and hostility in a sample of highly educated young adults. It became clear that high hostility subjects described their parents as more rejective and overprotective, and as providing less emotional warmth than low hostility subjects. Furthermore, in multivariate analyses perceived paternal rejection was found to be the strongest predictor of level of hostility in both men and women. A related study of the developmental precursors of TABP found that certain perceived parental characteristics, in particular rejection and lack of emotional warmth, were associated with TABP (Emmelkamp \& Karsdorp, 1987).

The present study, which was primarily designed to evaluate the association between hostility and myocardial infarction (MI; see Meesters \& Smulders, 1994; Meesters, Muris, \& Backus, 1995), enabled us to further investigate the developm mental aspects of hostility in a sample consisting of both MI patients and healthy neighbourhood control subjects. It was hypothesized that high hostility cases and controls would rate their parents as providing less emotional warmth and being more rejective than their low hostility counterparts. As in the previous study (Meesters, Muris, \& Esselink, 1995), no specific hypothesis was formulated regarding the relationship between perceived parental overprotection and indiviclual differences in hostility.

\subsection{METHOD}

\section{Subjects and procedures}

A total of 249 men from South Limburg, the southernmost region of The Netherlands, participated in this study. Cases were 81 consecutive patients, admitted to the two largest regional hospitals on account of a first clinically documented MI. Their mean age was 55.6 years ( $\mathrm{SD}=6.8$ ). The diagnosis of $\mathrm{MI}$ was based on the clinical history confirmed by a cardiologist, standard ECG readings, and plasma enzyme assessments. Patients suffering from another major disease, such as cancer, were excluded from participation.

MI patients were contacted when they were discharged from the coronary care unit. After explaining the aim and the procedure of the study, patients were asked to sign a written informed consent form. Next, they were asked to complete a set of questionnaires, including the Cook-Medley Hostility (Ho) Scale (Cook \& Medley, 1954) and the Buss-Durkee Hostility Inventory (BDHI; Buss \& Durkee, 1957). In addition, patients answered some questions about demographic characteristics and cardiovascular risk factors such as marital status, educational level, tobacco and 
coffee consumption, hypertension, diabetes mellitus, and family history of CHD. About four weeks after discharge from hospital patients were interviewed at home. This home interview comprised the Type A behaviour pattern (TABP) structured interview (SI; Rosenman, 1978) and the Maastricht Interview for Vital Exhaustion (MIVE) for the measurement of mental precursors of MI (see Meesters \& Appels, 1995a, 1995b). After these two interviews, the patient was finally requested to complete the Egna Minnen Beträffande Uppfostran (EMBU; Perris, Jacobsson, Lindström, von Knorring \& Perris, 1980), originally a Swedish paper-and-pencil test to measure perceptions of parental rearing behaviour. A complete data set was obtained on 81 MI cases.

Control subjects were 168 age-matched neighbourhood controls (NCs), who lived in the same street as the cases or in an adjacent street at the time of the cases' MI. Mean age of this group was 54.9 years $(S D=6.9)$. The neighbourhood control group was selected to obtain a reference group representing the source population (Miettinen, 1985). Control subjects were considered to be healthy, i.e. they had had no previously documented MI, coronary bypass surgery, or Percutaneous Transluminal Coronary Angioplasty (PTCA) and were not suffering from another major disease (e.g. cancer) at the time of the study. NCs were selected via the local telephone directory and were sent all above-mentioned questionnaires. NCs were also interviewed at home, exactly like the cases. This interview was scheduled about four weeks after completion of the questionnaires.

\section{Measures}

Both cases and controls were asked to complete the following self-report inventories:

The EMBU (Dutch version by Arrindell, Emmelkamp, Brilman, \& Monsma, 1983) is a 64-item self-report questionnaire to assess one's perception of parental rearing behaviour which was originally developed in Sweden by Perris and his colleagues (1980). All items are scored on a four-point Likert scale $(1=$ No, never; $2=$ Yes, but seldom; $3=$ Yes, often; $4=$ Yes, most of the time). Psychometric research has demonstrated that these items are distributed across four factors/scales: Rejection (25 items), Emotional Warmth (18 items), Overprotection (16 items), and Favouring Subject (5 items) (Arrindell et al., 1983). Three factors (Rejection, Emotional Warmth, and Overprotection) were found to have excellent cross-national invariance (Arrindell \& van der Ende, 1984; Arrindell et al., 1986) and were employed in the present study. Theoretically, scores may range between $25-100,18-72$, and 16-64 for Rejection, Emotional Warmth, and Overprotection, respectively. The internal consistencies of EMBU scales are all within acceptable limits, and all scales were found to be unrelated to social desirability, gender, age, and educational level (Arrindell et al., 1983). Furthermore, the results of the study by Arrindell et al. (1983) indicated that the retrospective nature of the EMBU did not have an unfavourable effect on the reliability and validity of the data due to recall errors or distorted accounts. In the 
present study, subjects answered each EMBU question by first assessing their father"s and then their mother's rearing behaviour.

The Dutch version of the Cook-Medley Hostility (Ho) Scale (Cook \& Medley, 1954) is a measure of hostility derived from the Minnesota Multiphasic Personality Inventory (MMPI). The Ho scale was originally constructed for the purpose of differentiating teachers with good rapport from those with poor rapport with their pupils. The Ho scale comprises 50 true-false items and scores range between 0 (not hostile at all) and 50 (extremely hostile). The scale displays satisfactory internal consistency, with Cronbach's $\alpha$ 's averaging about 0.80 (Smith \& Frohm, 1985). The coefficient $\alpha$ in the present sample was 0.85 . Ho scores are generally found to be stable over time in adult samples with two-year and four-year test-retest correlations of about 0.80 (Barefoot, Dahlstrom, \& Williams, 1983; Neissen, 1993; Shekelle, Gale, Ostfeld, \& Paul, 1983). Studies of the construct validity have indicated that cynicism and mistrust are the primary components of hostility as indexed by the Ho scale (Barefoot \& Lipkus, 1994; Greenglass \& Julkunen, 1989; Smith \& Frohm, 1985). Thus, persons with high scores on the Ho scale are best described as having a cynical, mistrustful attitude towards others, in other words, as highly hostile individuals who view their fellow-men as dishonest, ugly, mean, and asocial.

The Buss-Durkee Hostility Inventory (BDHI; Buss \& Durkee, 1957) is one of the most comprehensive measures of hostility. The scale comprises 66 items that assess seven distinct aspects of hostility (Assault, Indirect Hostility, Irritability, Negativism, Resentment, Suspicion, and Verbal Hostility). Furthermore, a total score or measure of general hostility can be derived by summing the seven hostility subscale scores. Several factor analytic studies have yielded two distinct factors, an Experiential Hostility factor, primarily constituted by the Resentment and Suspicion subscales, and an Expressive Hostility factor, primarily consisting of the Assault, Verbal Hostility, Indirect Hostility, and Irritability subscales (e.g. Bendig, 1962; Bushman, Cooper \& Lemke, 1991; Buss \& Durkee, 1957; Musante, MacDougall, Dembroski, \& Costa. 1989). Evidence with respect to the internal consistency and stability over time of the BDHII is limited. Biaggio, Supplee, and Curtis (1981) found 2-week test-retest relliability coefficients varying from 0.64 to 0.78 for the subscales, and 0.82 for the total score. In addition, Neissen (1993) reported two-year test-retest correlations for the subscales ranging from 0.44 to 0.72 , and 0.78 for the total score. Several studies have provided evidence in support of the construct validity of the scale by demonstrating positive associations with behavioural measures (e.g. shock administration), perception of violence, and expert ratings of aggressiveness (Matthews, Jamison, \& Cottington, 1985).

In the present study, a Dutch translation of the BDHI with a true-?-fallse response format was utilized. Factor analysis (principal components with VARIMAX rotation) of this Dutch form confirmed the distinction between the experiential and expressive components of hostility that was just mentioned. Furthermore, this instrument proved to have satisfactory internal consistency, with Cronbach"s $\alpha$ 's of $0.85,0.76$, and 0.85 
for the total BDHI score, BDHI Experiential hostility factor, and BDHI Expressive Hostility factor, respectively.

\section{Statistical analyses}

Questionnaire data were only included in the statistical analyses, if subjects had completely filled out both measures of hostility, i.e. the Ho scale and the BDHI, as well as the EMBU. This procedure yielded a final sample of 206 subjects $(67$ cases and 139 controls). In addition, the evaluation of the relationship between EMBU dimensions and hostility measures was restricted to the following measures: CookMedley Hostility scores and BDHI total scores to examine the relationship between EMBU dimensions and general hostility, and the Expressive and Experiential Hostility factors of the BDHI in order to assess the association between EMBU measures and different dimensions of hostility. All analyses were performed for cases and controls separately.

By means of t-tests the average scores of cases and controls on the three EMBU dimensions (i. e. rejection, emotional warmth and overprotection) were compared. To examine the association between EMBU subscales and individual differences in hostility, both the case group and the control group were divided into high and low hostility groups according to their scores on each separate hostility (sub)scale. This procedure was based on the median split of the control subsample, because the control group can be considered a more representative sample of the "normal" population than the MI case group. Lastly, to test whether the EMBU scalles accounted for unique sources of variance in hostility scores, a series of forward stepwise regression analyses was executed. In these analyses, the EMBU dimensions were the predictor variables and the separate hostility measures were the dependent variables.

\section{$5.4 \quad$ RESULTS}

\section{General findings}

Table 5.1 shows the internal consistency coefficients of the EMBU scales for both cases and controls. All Cronbach's $\alpha$ 's are within acceptable limits. Results displayed in table 5.1 further demonstrate that there are no significant differences between cases and controls in mean scores on any EMBU subscales. Results of additional paired t-tests are partially in line with the earlier findings of Perris at al. (1980), who showed that subjects rate their father's and their mother's behaviour differently. In the present sample, respondents perceive their mother as providing more emotional warmth and overprotection than their father, means being 47.9 vs. $50.5[\mathrm{t}(205)=$ $-5.32, p=0.000]$ and 33.4 vs. $36.3[t(205)=-7.43, p=0.000]$, respectively. With respect to the rejection subscale, no significant differences emerged, means being $37.3 \mathrm{vs} .37 .0[\mathrm{t}(205)=0.38, \mathrm{p}=0.71]$. 
Table 5.1. Reliability coefficients and mean scores (SDs) on EMBU measures for cases $(n=67)$ and controls $(n=139)$ separately

\begin{tabular}{lllll}
\hline & \multicolumn{2}{c}{ Crombach's $\alpha$} & \multicolumn{2}{c}{ Mean Score (SD) } \\
& Cases & Controls & Cases & Controls \\
\hline EMBU & & & & \\
& & & & \\
Of fathers & & & & \\
Rejection & 0.89 & 0.90 & $36.8(10.0)$ & $37.5(9.9)$ \\
Emotional warmth & 0.88 & 0.88 & $50.0(10.9)$ & $46.8(10.9)$ \\
Overprotection & 0.65 & 0.71 & $34.1(6.6)$ & $33.0(6.6)$ \\
Of mothers & & & & $37.1(9.8)$ \\
Rejection & 0.94 & 0.89 & $37.0(12.4)$ & $50.0(10.6)$ \\
Emotional warmth & 0.84 & 0.87 & $51.5(9.8)$ & $36.1(6.7)$ \\
Overprotection & 0.66 & 0.69 & $36.5(6.8)$ & \\
\hline
\end{tabular}

\section{Relationships between hostility measures}

Correlations of Cook-Medley (Ho) scores and the different dimensions of hostility as measured by the BDHI are presented in table 5.2 .

Table 5.2. Pearson product-monent correlations between the Cook-Medley Hostility (Ho) Scale and Buss-Durkee Hostility Inventory $(\mathrm{BDHI})$ measures for cases $(\mathrm{n}=67)$, controls $(\mathrm{n}=139)$, and total sample $(\mathbf{n}=206)$ separately

\begin{tabular}{lccc}
\hline & Cases & Controls & Total sample \\
\hline BDHI Total & 0.68 & 0.61 & 0.64 \\
Assault & 0.39 & 0.29 & 0.32 \\
Indirect Hostility & 0.36 & 0.32 & 0.33 \\
Irritability & 0.46 & 0.49 & 0.48 \\
Negativism & 0.44 & 0.40 & 0.42 \\
Resentment & 0.60 & 0.54 & 0.56 \\
Suspicion & 0.69 & 0.64 & 0.65 \\
Verbal Hostility & 0.36 & 0.25 & 0.28 \\
& & & 0.46 \\
Expressive factor & 0.50 & 0.44 & 0.69 \\
Experiential factor & 0.75 & 0.67 & \\
\hline
\end{tabular}

Note: For all correlations except for case group Assault, Indirect Hostality, and Verbal Hostility, $p<0.001$; for case group Assault, Indirect Hostility , and Verbal Hostility, $p<0.01$.

Although all correlations are statistically significant, the moderate strength of the correlations suggest that the Ho scale and the BDHI assess different aspects of the 
multidimensional hostility construct. The correlations of the Ho scale with $\mathrm{BDH}$ Resentment and Suspicion are considerable higher than the correlations with Assault, Indirect Hostility, and Verbal Hostility. This is further underlined by the finding that the relationship between Ho scores and the BDHI. Experiential Hostility factor is significantly stronger than the relationship with the BDHIl Expressive Hostility Factor $(r=0.69$ vs. $r=0.46, p=0.000)$.

\section{Association of Cook-Medley hostility and perceived parental rearing behaviour}

As can be seem in table 5.3 , highly hostile subjects in both the case group and the control group perceived their father and mother as giving less emotional warmth than low hostility subjects. In addition, high hostility control subjects reported having experienced higher levels of rejection by both father and mother. In cases, however, only the relationship between perceived rejection by the mother and hostility was significant. Finally, no significant differences in perceived parental overprotection between high and low hostility persons emerged among either cases or controls.

Table 5.3. Mean scores (SDs) on the EMBU subscales for high hostility and low hostility cases and controls, as defined by the Ho scale

\begin{tabular}{|c|c|c|c|c|}
\hline & \multicolumn{2}{|c|}{ Cases } & \multicolumn{2}{|c|}{ Controls } \\
\hline & \multicolumn{2}{|c|}{ Hostility } & \multicolumn{2}{|c|}{ Hostility } \\
\hline & $\begin{array}{l}\text { High } \\
(\mathrm{n}=38)\end{array}$ & $\begin{array}{l}\text { Low } \\
(n=29)\end{array}$ & $\begin{array}{l}\text { High } \\
(\mathrm{n}=69)\end{array}$ & $\begin{array}{l}\text { Low } \\
(n=70)\end{array}$ \\
\hline \multicolumn{5}{|l|}{ EMBU } \\
\hline \multicolumn{5}{|l|}{ Of fonters } \\
\hline Rejection & $38.2(10.7)$ & $34.9(8.8)$ & $39.5(10.8)$ & $35.7(8.7)^{*}$ \\
\hline Enotional warmeth & $47.4(11.6)$ & $53.4(9.3)^{*}$ & $44.8(11.9)$ & $48.8(9.6)^{*}$ \\
\hline Overprotection & $34.7(6.8)$ & $33.2(6.3)$ & $33.7(6.7)$ & $32.4(6.5)$ \\
\hline \multicolumn{5}{|l|}{ Of mothers } \\
\hline Rejection & $39.4(15.2)$ & $33.7(6.0)^{*}$ & $39.4(11.6)$ & $34.9(7.2)^{* * *}$ \\
\hline Emotional warmth & $48.7(10.6)$ & $55.2(7.2) * *$ & $47.7(12.1)$ & $52.3(8.5)^{*}$ \\
\hline Overprotection & $37.3(6.8)$ & $35.4(6.8)$ & $36.6(7.5)$ & $35.8(5.8)$ \\
\hline
\end{tabular}

Note: Differences befween high and low hostility groups were analysed with t-tests.

$* p<0.05 ; *$ * $p<0.01$

\section{Association of Buss-Durkee hostility and perceived parental rearing behaviour}

Results of t-test comparisons of high and low hostility individuals, as defined by various BDHI measures, are given in tables 5.4 to 5.6. Table 5.4 provides data with respect to $\mathrm{BDHI}$ total score, which is an index of general hostility. The results show 
that high hostility cases perceived both their father and their mother as more overprotective than low hostility cases. In the control group, no significant differences between high and low hostility subjects emerged with respect to perceived overprotection. Furthermore, high hostility controls described both their father and their mother as more rejective, whereas in cases this association was only found for rejection by mother. In the case group, subjects with high $\mathrm{BDHI}$ totall scores report less maternal emotional warmth than subjects with low BDHI total scores. High hostility controls indicated having experienced less emotional warmih from their fathers than low hostility controls.

Table 5.4. Mean scores (SDs) on the EMBU subscales for high hostility and low hostillity cases and controls, as defined by BDHII total scores

\begin{tabular}{|c|c|c|c|c|}
\hline & \multicolumn{2}{|c|}{ Cases } & \multicolumn{2}{|c|}{ Controls } \\
\hline & \multicolumn{2}{|c|}{ Hostility } & \multicolumn{2}{|c|}{ Hostility } \\
\hline & $\begin{array}{l}\text { High } \\
(\mathrm{n}=37)\end{array}$ & $\begin{array}{l}\text { Low } \\
(n=30)\end{array}$ & $\begin{array}{l}\text { High } \\
(n=69)\end{array}$ & $\begin{array}{l}\text { Low } \\
(n=70)\end{array}$ \\
\hline \multicolumn{5}{|l|}{ EMBU } \\
\hline \multicolumn{5}{|l|}{ Of fathers } \\
\hline Rejection & $38.4(10.7)$ & $34.8(8.9)$ & $40.1(10.4)$ & $35.0(8.8)^{* * *}$ \\
\hline Emotional warmtl & $49.2(10.7)$ & $51.0(11.5)$ & $43.9(12.1)$ & $49.7(8.9)^{\text {** }}$ \\
\hline Overprotection & $35.9(5.2)$ & $31.9(7.5)^{*}$ & $33.2(6.9)$ & $32.9(6.2)$ \\
\hline \multicolumn{5}{|l|}{ of mothers } \\
\hline Rejection & $39.9(15.3)$ & $33.4(5.9)^{*}$ & $39.6(12.1)$ & $34.6(5.9)^{* *}$ \\
\hline Emotional warmth & $49.4(10.8)$ & $54.1(7.9)^{*}$ & $48.4(11.8)$ & $51.7(9.1)$ \\
\hline Overprotection & $38.2(5.5)$ & $34.4(7.7)^{*}$ & $36.5(7.3)$ & $35.8(5.9)$ \\
\hline
\end{tabular}

Note: Differences between high and low hostility groups were analysed with t-tests.

* $\mathrm{p}<0.05 ; * \mathrm{p}<0.01$

Means and standard deviations on EMBU scales for both high and low hostility cases and controls, as measured by the BDHI Expressive factor and the BDHI Experiential factor are displayed in table 5.5 and table 5.6, respectively. Especially in the sample of control subjects, the strongest relationships between hostility and perceived parental rearing styles were observed for experiential hostility. Cases who scored high on the expressive hostility factor reported more perceived overprotection from both father and mother and viewed their mother as more rejective than cases with low scores on this factor (see table 5.5). In the sample of controls, lack of emotional warmth from the father proved to be the only parental characteristic that discriminated between subjects with high scores and subjects with low scores on expressive hostility. 
Chapter 5

Table 5.5. Mean scores (SDs) on the EMBU subscales for high hostility and low hostility cases and controls, as defined by the BDHI Expressive factor

\begin{tabular}{|c|c|c|c|c|}
\hline & \multicolumn{2}{|c|}{ Cases } & \multicolumn{2}{|c|}{ Controls } \\
\hline & \multicolumn{2}{|c|}{ Hostility } & \multicolumn{2}{|c|}{ Hostility } \\
\hline & $\begin{array}{l}\text { High } \\
(n=34)\end{array}$ & $\begin{array}{l}\text { Low } \\
(n=33)\end{array}$ & $\begin{array}{l}\text { High } \\
(n=69)\end{array}$ & $\begin{array}{l}\text { Low } \\
(n=70)\end{array}$ \\
\hline \multicolumn{5}{|l|}{ EMBO } \\
\hline \multicolumn{5}{|l|}{ Of fathers } \\
\hline Rejection & $37.5(9.3)$ & $36.1(10.8)$ & $39.1(9.0)$ & $36.0(10.6)$ \\
\hline Emotional warmth & $49.6(10.7)$ & $50.5(11.5)$ & $44.9(11.1)$ & $48.7(10.6) *$ \\
\hline Overprotection & $36.0(5.0)$ & $32.1(7.4)^{*}$ & $33.1(7.1)$ & $33.0(6.0)$ \\
\hline \multicolumn{5}{|l|}{ of mothers } \\
\hline Rejection & $40.3(15.9)$ & $33.5(5.7)^{2 / 2}$ & $38.7(10.9)$ & $35.5(8.4)$ \\
\hline Enotional warmth & $49.6(10.9)$ & $53.4(8.1)$ & $49.1(11.0)$ & $51.0(10.2)$ \\
\hline Overprotection & $38.2(5.5)$ & $34.7(7.6)^{*}$ & $36.2(7.4)$ & $36.1(5.9)$ \\
\hline
\end{tabular}

Note: Differences between high and low hostility groups were analysed with t-tests.

$* \mathrm{p}<0.05$

Table 5.6. Mean scores (SDs) on the EMBU subscales for high hostility and low hostility cases and controls, as defined by the BDHI Experiential factor

\begin{tabular}{|c|c|c|c|c|}
\hline & \multicolumn{2}{|c|}{ Cases } & \multicolumn{2}{|c|}{ Controlls } \\
\hline & \multicolumn{2}{|c|}{ Hostility } & \multicolumn{2}{|c|}{ Hostility } \\
\hline & $\begin{array}{l}\text { High } \\
(n=45)\end{array}$ & $\begin{array}{l}\text { Low } \\
(n=22)\end{array}$ & $\begin{array}{l}\text { High } \\
(n=72)\end{array}$ & $\begin{array}{l}\text { Low } \\
(n=67)\end{array}$ \\
\hline \multicolumn{5}{|l|}{ EMBU } \\
\hline \multicolumn{5}{|l|}{ Of fawers } \\
\hline Rejection & $38.3(11.3)$ & $33.6(5.7)^{\text {* }}$ & $39.7(10.4)$ & $35.3(8.9)^{* * *}$ \\
\hline Emotional warmth & $48.5(11.3)$ & $53.2(9.9)$ & $43.6(12.4)$ & $50.3(7.9)^{*}$ \\
\hline Overprotection & $35.1(6.8)$ & $32.0(5.7)$ & $32.9(6.1)$ & $33.2(7.0)$ \\
\hline \multicolumn{5}{|l|}{ Of mothers } \\
\hline Rejection & $38.0(13.0)$ & $34.8(11.1)$ & $39.2(12.1)$ & $34.7(5.9)^{* * *}$ \\
\hline Emotional warmth & $50.6(9.9)$ & $53.2(9.4)$ & $46.8(11.8)$ & $53.5(7.9)^{* * * *}$ \\
\hline Overprotection & $37.6(6.8)$ & $34.1(6.2)^{*}$ & $36.1(7.1)$ & $36.2(6.2)$ \\
\hline
\end{tabular}

Note: Differences between high and low hostility groups were analysed with t-tests.

$* p<0.05 ; * * 00.01 ; * * *<<0.001$

As is shown in table 5.6, cases scoring high on the experiential hostility factor described their father as more rejective and their mother as more overprotective than 
cases scoring low on this factor. All other associations of EMBU dimensions and experiential hostility failed to be significant. Yet, high hostility control subjects (as defined by the BDHI Experiential hostility factor) perceived their father and mother as being more rejective and providing less emotional warmth than low hosthity controls.

\section{Multivariate analyses}

In order to inwestigate whether perceived parental rearing characteristics account for unique sources of variance in hostility, a series of (forward) stepwise regression analyses was carried out for cases and controls separately. In these analyses, the EMBU subscales Rejection, Emotional Warmth, and Overprotection were included as the predictors and the above-mentioned hostility measures as the dependent variables. The results are summarized in table 5.7 (case group) and table 5.8 (control group). As can be concluded from these tables, in 11 out of 16 regression equations Rejection entered in the first step, explaining a significant part of the variance in hostility, whereas in 3 out of 16 equations Emotional Warmth did so. Overprotection, however, did not enter in the first step in any of the equations.

Table 5.7. Main results of the series of forward stepwise regression analyses with EMBU subscales being the predictors and hostility measures being the dependent variables for cases $(\mathrm{n}=67)^{\mathrm{I}}$

\begin{tabular}{|c|c|c|c|c|c|}
\hline Dependent & Predictor $(s)^{2}$ & (Partial) r & $t$ & p-value & $\%$ variance $^{3}$ \\
\hline \multicolumn{6}{|l|}{ Of fathers } \\
\hline \multirow[t]{2}{*}{ Ho scale } & 1. Emotional warmin & -0.31 & 2.6 & $<0.05$ & 10 \\
\hline & 2. Overprotection & 0.26 & 2.2 & $<0.05$ & 16 \\
\hline BDHI Total & 1. Rejection & 0.28 & 2.4 & $<0.05$ & 8 \\
\hline Expressive hostility & 1. Rejection & 0.26 & 2.1 & $<0.05$ & 7 \\
\hline Experiential hostility & no variables entered & & & & \\
\hline \multicolumn{6}{|l|}{ Of mothers } \\
\hline Ho scale & 1. Rejection & 0.40 & 3.5 & $<0.001$ & 16 \\
\hline BDHI Total & 1. Rejection & 0.34 & 3,0 & $<0.01$ & 12 \\
\hline Expressive hostility & 1. Rejection & 0.33 & 2.9 & $<0.01$ & 11 \\
\hline Experiential hostility & no variables entered & & & & \\
\hline
\end{tabular}

\section{Note:}

'Only the results of the analyses in which predictor(s) entered in the regression are presented.

${ }^{2}$ The predictor variables that entered in the stepwise regression are numbered.

${ }^{\text {"Cumulative. }}$ 
In the case group, perceived (lack of) emotional wamth from the father and perceived rejection by the mother explained significant proportions of the variance in Cook-Medley Ho scores. Furthermore, perceived overprotection by the father accounted for an extra $6 \%$ of the variance in Ho scores. Perceived rejection by both father and mother accounted for significant proportions of variance in BDHI Total and BDHI Expressive Hostility scores.

With respect to the sample of controls, table 5.8 shows that perceived rejection by both father and mother accounted for significant proportions of the variance in scores on the Ho scale, BDHI Total, and BDHI Expressive Hostility factor, respectively. In addition, lack of emotional warmth from father accounted for a significant part of the variance in scores on the BDHI Experiential Hostility factor. As to the relationship between perceived characteristics of the mother and expressive hostility, emotional warmth from the mother also entered in the first step, whereas overprotection accounted for an extra $3 \%$ of variance.

Tablle 5.8. Main results of the series of forward stepwise regression analyses with EMBU subscales being the predictors and hostillity measures being the dependent variables for controls $(\mathrm{n}=139)^{\mathrm{t}}$

\begin{tabular}{|c|c|c|c|c|c|}
\hline Dependent & Predictor $(\mathrm{s})^{2}$ & (Partial) $r$ & t & $p$-value & $\%$ variance $^{3}$ \\
\hline \multicolumn{6}{|l|}{ Of fathers } \\
\hline Ho scale & 1. Rejection & 0.29 & 3.5 & $<0.001$ & 8 \\
\hline BDHI Total & 1. Rejection & 0.27 & 3.3 & $<0.01$ & 7 \\
\hline Expressive hostility & 1. Rejection & 0.21 & 2.5 & $<0.05$ & 4 \\
\hline Experiential hostility & 1. Emotional warmth & -0.30 & 3.7 & $<0.001$ & 9 \\
\hline \multicolumn{6}{|l|}{ of mothers } \\
\hline Ho scale & 1. Rejection & 0.28 & 3.4 & $<0.001$ & 8 \\
\hline BDHI Total & 1. Rejection & 0.28 & 3.5 & $<0.001$ & 8 \\
\hline Expressive hostility & 1. Rejection & 0.23 & 2.8 & $<0.01$ & 5 \\
\hline \multirow[t]{2}{*}{ Experiential hostility } & 1. Emotional warmth & -0.27 & 3.3 & $<0.01$ & 7 \\
\hline & 2. Overprotection & 0.18 & 2.1 & $<0.05$ & 10 \\
\hline
\end{tabular}

Note:

"Only the results of the analyses in which predictor(s) entered in the regression are presented.

2 The predictor variables that entered in the stepwise regression are numbered.

${ }^{3}$ Cunnulative. 


\subsection{DISCUSSION}

In summary, the present findings suggest that perceived parental rearing behaviour accounts for significant proportions of variance in hostility in a sample of male myocardial infarction patients as well as in a sample of male healthy control subjects. Although the associations observed in the case group and in the control group are not fully identical, the patterns of results are ciearly similar. By and large, high hostility was associated with perceived parental rejection and perceived lack of emotional warmth in both cases and controls. The findings specifically suggest that perceived rejection by both parents is most strongly related to level of general hostility (as defined by Ho scale and BDHI Total scores). In addition it was found that rejection by both father and mother seems to be related to the expressive aspects of hostility in particular, while perceived lack of parental emotional warmth seems to be most strongly associated with experiential hostility components. The influence of the parental characteristic of overprotection on the development of hostility, in contrast, seems to be negligible. Furthermore, the results of the present study generally corroborate the findings of our previous study (Meesters, Muris \& Esselink, 1995), in which especially rejection and lack of emotional warmth were found to be predictive of an individual's level of hostility. It should be noted that a sample of highly educated, healthy young (mean age 21.1 years) adults was examined in that study, whereas the present study sample consisted of middle-aged subjects mainly with little education.

The findings of the current investigation are also in line with the results of a few other studies that explored the early familial antecedents of hostile characteristics and behaviours (Houston \& Vavak, 1991; Smith et al., 1988; Woodlall \& Matthews, 1989). These studies all suggest that unfavourable early family contexts, described as unaccepting, unsupportive, high in conflict and rigidly and harshly controlled, contribute to the development of hostility. However, apart from the interpretation of these findings from the point of view of social learning, the possibility of genetic determinants of hostility should also be taken into consideration. The role of heritability in the development of hostile attitudes and behaviours may be explained in two different ways. Firstly, children may inherit a predisposition to observe and respond to parental upbringing behaviour, such as the behaviours examined in the present study, in a way that promotes the development of hostility. Secondly, children may inherit certain behavioural and emotional characteristics that easily provoke their parents to behave as described above (e.g. rejective, punitive and/or hostile), which will reinforce the development of hostility. At present, evidence generated by behavioural genetics research suggests a reciprocal relationship between genetically based psychological characteristics and environmental factors (Plomin \& Bergeman, 1991; Scarr \& McCartney, 1983).

Although the findings of the present study are consistent with previous research into the possible influence of parental attitudes and behaviours on hostility, it must be stressed that our study has several limitations. First, the measures of parental child- 
rearing practices and attitudes used in this study were based on subjects" retrospective accounts rather than on assessments of actual parental rearing behaviour. Therefore, it is not unlikely for hostile individuals to have negative recollections of their early childhood because of their negative attitude toward other people in general and their parents in particular. Still, the present findings are similar to those of Woodall and Matthews (1989) who examined angry and hostile children concurrently. At the same time, memories of early family experiences and events were found to be generally accurate and reliable in a study by Robins et al. (1985). Furthermore, recent results from a 4-year longitudinal sudy of hostile characteristics in children suggest that the degree of support and conflict in the family may indeed influence the development of hostility (Woodall \& Matthews, 1993). The second limitation was that the study sample did not include any female subjects. For this reason, it would be unwise to generalize findings to the population as a whole. Future investigations of the developmental origins of hostility should also include women. Third, in this study, hostility was measured by means of self-report questionnaires. Further research, relating (perceived) parental rearing characteristics to behavioural ratings of hostility, would be useful.

In conclusion, the findings of the present study support the idea that family environment factors play an important part in the development of psychological characteristics (i.e. hostility) in children that may place them at risk for CHD in later life. To be more specific, our findings suggest that the expressive components of hostility in adult males are most strongly associated with descriptions of parents as having been more rejective, whereas perceived lack of emotional warmth was found to be more closely related to the experiential aspects of hostility. Admittedly, no direct relationship was found between EMBU measures and CHD. That is to say, cases and controls did not differ with respect to memories of their parents' rearing behaviour. The relationship between family environment factors, such as rearing behaviour, and CHD is apparently indirect. To gain a better understanding of the aetiological antecedents of hostility, these results will have to be replicated and expanded in additional longitudinal research. A better insight into this matter could further the development of intervention programs to prevent or modify the development of hostile attitudes and behaviours in children, thereby leading to a reduced risk of CHD in later life. 


\section{CHAPTER 6}

\section{AN INTERVIEW TO MEASURE VITAL EXHAUSTION. I. DEVELOPMENT AND COMPARISON WITH THE MAASTRICHT QUESTIONNAIRE ${ }^{1}$}

\subsection{SUMMARY}

This article presents an interview designed to measure vital exhaustion (VE), a state characterized by unusual fatigue, increased irritability and feelings of demoralization, which has been found to be a short-term risk indicator of myocardial infarction (MI). This state can be measured by means of the Maastricht Questionnaire (MQ). However, the MQ was found to have some major shortcomings for the selection of subjects for laboratory or clinical investigations. For this reason we designed an interview to assess VE. To test the hypothesis that the interview registers prodromal feelings of MI, the interview was administered to a sample of 81 males who were hospitalized because of a first myocardial infarction (mean age 55.6 years; sd 6.8) and a sample of 168 healthy males (mean age 54.9 years; sd 6.9). Mean scores of cases and controls differ significantly $(7.8$; sd 6.0 and 3.6 ; sd 4.0 , respectively; $t=5.65 ; \mathrm{p}=0.000$ ). Mean scores on the questionnaire discriminated significantly too, but the discriminating power was much lower $(t=2.31 ; p=0.02)$. The article gives detailed instructions on the way in which the interview should be administered and scored.

\subsection{INTRODUCTION}

Feelings of excess fatigue and lack of energy have been found to constitute the most prevalent precursor of myocardial infarction (MI) and sudden death (SD). Estimates of the number of subjects who experienced feelings of tiredness or general malaise before MI or SD range between $35 \%$ and $60 \%$ (Alonzo, Simon, \& Feinleib, 1975; Fraser, 1978; Kinlen, 1973; Kuller, 1978; Rissanen, Romo, \& Siltanen, 1978). Despite this high prevalence, few studies have addressed the nature and origins of these feelings.

Cardiological textbooks give different explanations of the causes of tiredness preceding MI and SD, such as left ventricular dysfunction leading to inadequate

\footnotetext{
${ }^{1}$ Meesters, C., \& Appels, A. (1995). An interview to measure vital exhaustion. I. Development and comparison with the Maastricht Questionnaire. Psychology and Health, in press.
} 
cardiac output, heart failure, depression, side effects of cardiovascular drugs (particularly beta-adrenergic blocking agents and diuretics), anxiety, angina pectoris, sleep problems, and prolonged psychological tension (Brandenburg, Fuster, Giuliani, \& McGoon, 1987; Braunwald, 1988; Hurst, Schlant, Rackley, Sonnenblick, \& Wenger, 1990). These factors may all be associated with tiredness. However, we do not know whether these factors sufficiently explain why feelings of excess fatigue constitute the most prevalent premonitory symptom or precursor of $\mathrm{ML}$ or why they are associated with future manifest heart disease in apparently healthy individuals. The actual physiological mechanism underlying the fatigue associated with future heart disease is unknown.

The large number of explanations indicates that the unusual tiredness preceding MI and SD still is a phenomenon that is insufficiently understood. Further progress requires an instrument to measure these feelings as accurately as possible. If future research were to show that excess fatigue is a marker of (sub)clinical heart disease, such an instrument could have some diagnostic value and might contribute to the identification of subjects at elevated risk. If future research were to show that unusual tiredness reflects a pathogenic process in which a poor cardiac condition interacts with a distressed mental state resulting in a progressive downward spiral, proper assessment of this excess tiredness might contribute to gaining a deeper insight into that process. It might also be useful for the investigation of the similarities and dissimilarities between pre-myocardial infarction fatigue and other fatigue states, such as the chronic fatigue syndrome.

When we initiated the attempt to measure the mental precursors of MI we assumed that the feelings of fatigue and loss of energy reflect the exhaustion stage of the General Adaptation Syndrome, as described by Selye (Selye, 1967). We also had the feeling that premature item selection should be avoided because the nature of the prodromal feelings was not yet well-documented. Therefore, we did not adopt the parsimonious strategy of selecting an established psychiatric or psychological instrument and testing its predictive power in a longitudinal study. Instead, we interviewed a large number of coronary victims and their wives, asking them about the feelings and behavioral changes experienced in the year preceding the coronary event and used their reports to construct an item pool, which would be evaluated in epidemiological studies.

During the interviews, the majority of the patients said that they had experienced fatigue, listlessness and loss of energy before their infarction, while the wives often stressed increased irritability as the major behavioral change they had noticed in their husbands. Many patients also reported that they had felt frustrated or defeated by some form of injustice afflicted upon them. Many of these symptoms seemed to indicate depression. However, mood disturbances (feeling sad, blue, depressed), a lowered selfesteem and feelings of guilt were usually absent. Therefore, we labeled this cluster of complaints as "vital exhaustion" (VE) and defined it as a state characterized by 1) unusual fatigue and lack of energy, 2) increased irritability and 3) feelings of demoralization. 
The interviews of the coronary patients were used to construct an item pool by rephrasing the self-descriptions of the patients into questions. Sixty-three questions were formulated. This item pool was subsequently tested in two studies. Thirty-seven items were found to discriminate between coronary cases and healthy controls (Appels, 1980; Verhagen, Nas, Appels, van Bastelaer, \& Winnubst, 1980). These items were labeled as form $A$ of the Maastricht Questionnaire (MQ) in order to give the item pool a neutral name.

Further examination of the concept of vital exhaustion would have been unwarranted if the reports of the coronary victims had been strongly influenced by hindsight or a search for meaning after the catastrophe or if these feelings could simply be interpreted as a side effect of manifest heart disease, medication or the classic risk factors. The hypothesis that feelings of VE precede MI had to be tested in a prospective study.

A longitudinal study was started in 1979, when the Municipal Health Authority of the city of Rotterdam (The Netherlands) started a health check-up of city employees. This study was labeled the Rotterdam Civil Servants Study (RCSS). Between January 1979 and December 1980, 3,877 male employees, aged between 39 and 65 participated. The medical examination included measurements of blood pressure, serum cholesterol, glucose tolerance, relative weight, smoking and an assessment of angina pectoris, using the London School of Hygiene Cardiovascular Questionnaire (Rose, Blackburn, Gillum, \& Prineas, 1982). A resting electrocardiogram completed the cardiovascular screening. All subjects completed form A of the MQ. Twenty-one new items, derived from a new series of clinical interviews, were added to this scale. So the questionnaire used in the RCSS consisted of 58 (37 "old" and 21 "new") items.

The cohort was followed during an average period of 4.2 years. Among those free of CHD at screening 21 subjects died of MI, while well-documented non-fatal MI occurred in 38 subjects. The relative risk of fatal or non-fatal MI associated with a score above the median of the 37-item form $A$ of the $M Q$, controlling for age, cholesterol, smoking and blood pressure, was $2.67(\mathrm{p}<0.01)$. An analysis of the observed association against length of time interval showed a sharp decrease in the relative risk of events occurring in the first, second, third or fourth year of follow-up. The age-adjusted risk for the first year of follow-up was 10.05, while the age-adjusted risks for the following years were $2.23,3.04$ and 0.68 , respectively. These data strongly suggest that exhaustion is a short-term risk indicator. Elevated scores at screening were not observed among future cancer or gastrointestinal patients. This suggests that VE is related to future CHD only (Appels \& Mulder, 1988; Appels \& Schouten, 1991).

Item analysis of the form A of the MQ showed that the predictive power was based upon 16 items. Eight of the 21 iterns added to the scale were predictive. On the basis of this item analysis the final form of the MQ (form B) was constructed by selecting the predictive items. Three items were omitted because there were some doubts about their validity. Thus, the final form consists of 21 items (Appels, Höppener, \& Mulder, 1987). 
The answer categories of each item are Yes,?, or No. Each confirmation of a complaim is coded as 2 , all question marks are coded as 1 , and a negative answer is coded as 0 . Therefore, the range of the scores is $0-42$. In the RCSS, the mean score of form $B$ was found to be 8.82 . The Standard Deviation was 8.67 . These statistics might be used to compute a cutting point to divide subjects into "exhausted" and "nonm exhausted" subgroups. Such a cutting-point might be formed by a score which is equal to or higher than one standard deviation above the mean score. This corresponds to a score of 17 or higher.

Form $B$ of the MQ has been used in several case-control studies. The most extensive was carried out by Falger (1989), whose study compared 133 male MI-cases with 133 neighbourhood controls and 192 hospital controls. The second group was included in order to control for the effects of hospitalization on the recall of feelings of exhaustion. By dividing cases and controls into two groups, exhausted and non-exhausted subjects, on the basis of a score above or below the median of the MQ (i.e. eight affirmative replies), Falger estimated the relative risk of first $\mathrm{MI}$ assocjated with exhaustion at $7.35(95 \% \mathrm{CI} 4.3-13.3)$ in the neighbourhood series and at 2.90 (95\% CI $1.7-4.9)$ in the hospital series. These data showed that feelings of exhaustion constitute a risk indicator of MI. They also showed that elevated exhaustion scores are also present in other patient groups. Three interpretations of this finding were offered: VE is a precursor of diseases other than CHD; other diseases are associated with one or more symptoms of exhaustion (e.g. sleep problems); or recent hospitalization may have caused some retrospective bias. A study by Nelissen-de Vos showed that the second interpretation is to be preferred. Painful ailments may cause somewhat elevated MQ scores (Nelissen-de Vos, 1994). A case-control study comparing 79 women who had been hospitalized because of a first MI with 90 women who had been hospitalized in the general surgery and orthopaedic surgery units of the same hospitals showed that female victims of $\mathrm{MI}$ reported significantly more symptoms of exhaustion than controls, the estimated relative risk of exhaustion, adjusted for age, hypertension, smoking, and non-anginal pain being 2.75 (95\% Cl $1.3-5.9$ ) (Appels, Falger, \& Schouten, 1993). Therefore, it may be concluded that VE is a risk indicator of MI in both genders.

Because all males who had experienced an $\mathrm{MI}$ in the past or who suffered from angina pectoris were excluded from the analyses of the data of the RCSS, it could be nlled out that the risk of vital exhaustion was confounded by manifest heart disease. However, the RCSS and the case-control studies could not rule out the possibility that VE is predictive of MI because this state reflects subclinical heart disease.

To gain insight into the question whether VE is a marker of subclinical heart disease Kop investigated the association between VE (as measured by form $B$ of the MQ) and the extent of atherosclerosis in 127 angioplasty patients, the changes in exhaustion scores before and after angioplasty, and the clinical course after successful angioplasty among exhausted and non-exhausted patients. The occurrence of new cardiac events was recorded during a 1.5 year follow-up. Kop observed a significant association between the number of diseased vessels and $\mathrm{MQ}$ scores both before and after 
angioplasty. However, the explained variance was of low magnitude only (4\%). No association was observed between VE and left ventricular ejection fraction.

Angioplasty resulted in a decrease in feelings of exhaustion. However, $60 \%$ of the patients were still exhausted after revascularization. During follow-up, a new cardiac event (defined as either cardiac death, $\mathrm{MI}$, coronary bypass surgery, repeated Percutaneous Transluminal Coronary Angioplasty (PTCA), restenosis of a dilated artery, or new angina with documented ischemia) occurred in 29 patients. VE was significantly associated with a new cardiac event after controlling for remaining stenoses after PTCA, the extent of vessel disease and other relevant clinical characteristics (Kop, Appels, Mendes de Leon, de Swart, \& Bär, 1994).

Although both prospective studies (i.e. Appels \& Mulder, 1988; Kop et al., 1994) indicate that the MQ has predictive validity, its value for the selection of subjects for laboratory or clinical investigations is questionable because it has some major shortcomings. Highly elevated scores are not necessarily indicative of a breakdown in adaptation resulting from prolonged overwork or long-standing problems that could not be solved. They may also reflect a general tendency to complain or longstanding mental, or somatic problems. This would cause a decrease in the specificity of the assessment. The measurement cannot be corrected if a subject misinterprets a question and it does not leave room for registration of idiosyncratic expressions. Finally, the questionnaire hardly registers important qualitative characteristics or does not register them at all. These shortcomings were discovered by van Diest during the preparation of a sleep physiological study of exhausted and non-exhausted males. Because he needed carefully selected samples, he asked several experts to interview his questionnaire-based samples of 15 exhausted and 10 non-exhausted males. Two of the 15 exhausted males were found to suffer from a serjous somatic disease, three were found to be unrepresentative because their complaints did not reflect a breakdown in adaptation to prolonged stress because of their chronic nature, and one subject was not exhausted at all and was therefore included in the group of non-exhausted males (van Diest \& Appels, 1994). This experience made us decide to develop a structured interview for use in non-epidemiological studies. In order to give the interview a neutral name, we labeled it the Maastricht Interview for Vital Exhaustion (MIVE).

In the following sections we will first describe the construction of the interview and the scoring of the answers. Second, we will describe findings of a case-control study: the percentages of cases and healthy controls who endorsed an item, to provide insight into the occurrence of each prodromal complaint. Third, the correspondence between the interview and the $\mathrm{MQ}$ will be described. Fourth, the question will be addressed whether the interview discriminates better between cases and controlls than the questionnaire. If the interview had less or equal power in discriminating cases from controls than the $\mathrm{MQ}$, its use as an instrument for selecting subjects for experimental studies would be questionable. Information about the reliability and validity of the interview will be presented in the companion paper (Meesters \& Appels, 1995b). 


\subsection{CONSTRUCTION OF THE INTERVIEW, FORMAT AND SCORING}

The core of the interview is formed by nineteen items of form $\mathrm{B}$ of the MQ. One item, asking about difficulty falling asleep, was removed. This item was only weakly predictive of MI in the RCSS (Appels \& Mulder, 1988) and did not discriminate in the case-control study by Nelissen-de Vos (1994). We also removed the item "I feel fine", because it did not fit into the new format very well. Two items asking about increased irritability were added because of their very strong discriminating power in the casecontrol studies. We also addled the question "Do you shrink from your regular work as if it were a mountain to climb?". This item was found to be predictive of MI in the prospective study, but was not included in form $B$ of the $M Q$, because subjects without work were found to experience some difficulty answering that question. Therefore, this item was adapted slightly. Finally, we split the item "Do you feel dejected or defeated?" into two separate questions so as to cover this aspect of VE by more questions. Thus, the interview has 23 questions. It is given in appendix $I$.

The interview starts by asking fairly general questions about the three aspects of vital exhaustion: loss of energy, increased irritability and feelings of demoralization. The first question is followed by a question about the duration of the complaint. This additional question is repeated after each following question until the duration of the state of exhaustion becomes clear. The items are scored dichotomously as either absent or present. A question mark is added to each item, which may be used when the interviewer is unable to decide whether the complaint is absent or present. Items scored as "?" during the interview are repeated at the end of the interview. In case the answer remains unclear the complaint is scored as absent.

The interview can be used to obtain either a categorical or a dimensional classification. The dimensional classification considers exhaustion as a continuum, ranging between a minimum score of 0 and a maximum score of 23. The dimensional classification can be converted into a categorical classification by using a cut-off score. We recommend placing the cut-off point between 7 and 8 . This cut-off point corresponds to the mean score plus one standard deviation of the questionnaire used in the RCSS (Appels \& Mulder, 1988).

Appendix II gives additional instructions on the way in which the interview should be administered and scored. One of these recommendations is to exclude subjects who have experienced feelings of exhaustion for more than 18 months from experimental studies. Longstanding complaints of exhaustion may reflect stable personality characteristics or longstanding adverse somatic or psychiatric conditions, such as pain, the chronic fatigue syndrome or depression. It is the aim of the interview to assess a relatively recent breakdown in adaptation to stress. Inclusion of subjects whose complaints lasted for more than 18 months in an experimental study would make the "index-group" less representative of the "target-group" formed by subjects who are at elevated risk for myocardial infarction. 


\subsection{THE OCCURRENCE OF EXHAUSTION BEFORE MYOCARDIAL INFARCTION: EMPIRICAL DATA}

Data regarding the occurrence of feelings of exhaustion before MI were collected in a case-control study. Altogether, 249 males aged between 35 and 65 from the South Limburg region of the Netherlands took part in the study. The cases were 81 patients (mean age 55.6 years, standard deviation 6.8 years) who were hospitalized for a first documented $\mathrm{MI}$ in the cardiology units of the two largest regional hospitals between January 1990 and December 1990; they constinted a consecutive series. Every final diagnosis of first MI was based on the clinical history recorded by a cardiologist, on standard ECG readings, and on maximum plasma enzyme assessments. Patients with other serious diseases (e.g. cancer) were excluded from the study.

Once a week all new cases with a first MI were visited by the first author. These patients had recently been discharged from the coronary care unit. After the purpose of the study had been explained, the patient was requested to sign an informed consent form and was given a set of questionnaires. This set of self-report questionnaires included the retrospective version of form B of the Mastricht Questionnaire (MQ) (Appels et al., 1987), the Cook-Medley Hostility Scale (Cook \& Medley, 1954), the Buss-Durkee Hostility Inventory (Buss \& Durkee, 1957), and the London School of Hygiene Cardiovascular Questionnaire (Rose et al., 1982) for the measurement of angina pectoris within the last six months (prior to $\mathrm{MI}$ ). Variables such as marital status, educational level, smoking, coffee consumption, high blood pressure, diabetes mellitus, and family history of CHD were also included. These instruments will be described in the companion paper (Meesters \& Appels, 1995b). Patients were asked to complete the questionnaires as soon as possible and to return them within two weeks after discharge from hospital. About two weeks after discharge from hospital the first author phoned each patient to make an appointment for an interview at home. In all, 94 MI cases were invited to participate, 13 of whom refused. The final response rate was $86 \%$.

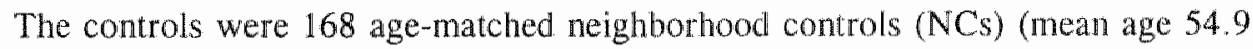
years, standard deviation 6.9 years). These persons lived in the same street as the MIcases or in an adjacent street at the time of the latters" cononary event. "The reason for selecting control subjects residing in the same neighborhood was the wish to compose a reference group representative of the source population (Miettinen, 1985). People with a previously documented MI or afficted with a serious illness (e.g. cancer) at the time were excluded from participation. NCs were selected from the telephone directory and were subsequently mailed the same set of questionnaires between January 1990 and May 1991. On receipt of the questionnaires, NCs were phoned by the first author in order to make an appointment for an interview at home. In all, 204 eligible controls were invited to participate in the study, 168 of whom eventually did so (82\%).

Percentages of cases and controls who endorsed an item of the interview are presented in table 6.1. The table shows that feelings of tiredness, irritability and 
annoyance were the most prevalent complaints. All complaints, except four, were more prevalent among cases. The non-discriminating questions reflect cognitive impaiment, loss of libido and crying spells. These items were predictive of $\mathrm{MI}$ in the RCSS (Appels \& Mulder, 1988) and discriminated significantly between cases and controls in the study by Falger (1989). Therefore, we are inclined to atribute this lack of discriminating power to sample fluctuations and did not consider removing these items from the interview.

Table 6.1. Percentages of positive answers of cases and controls to each question of the interview

\begin{tabular}{|c|c|c|c|c|c|}
\hline item & content & $\begin{array}{l}\text { cases } \\
(n=80)\end{array}$ & $\begin{array}{l}\text { controls } \\
(n=167)\end{array}$ & $x^{2}$ & \\
\hline 01 & tired & 60.0 & 32.9 & 16.30 & $* * *$ \\
\hline 02 & لistless & 42.5 & 12.0 & 29.50 & 橉*** \\
\hline 03 & lack of energy & 45.0 & 13.2 & 30.49 & $* *$ \\
\hline 04 & irritability & 57.5 & 30.5 & 16.49 & $* * *$ \\
\hline 05 & dejected & 22.5 & 7.8 & 10.67 & $* *$ \\
\hline 06 & empty battery & 45.0 & 28.7 & 6.37 & $*$ \\
\hline 07 & inability to accomplish & 37.5 & 19.2 & 9.68 & *** \\
\hline 08 & waking up exhausted & 37.5 & 15.6 & 14.84 & 级米米 \\
\hline 09 & disrupted sleep & 38.8 & 20.4 & 9.43 & *** \\
\hline 10 & inability to cope & 46.3 & 16.8 & 24.25 & $* * *$ \\
\hline 11 & give up trying & 27.5 & 10.2 & 12.20 & $* * *$ \\
\hline 12 & annoyed & 50.0 & 24.6 & 15.89 & $* *$ \\
\hline 13 & blow up & 40.0 & 21.0 & 9.92 & $* *$ \\
\hline 14 & decrease in energy & 31.3 & 6.0 & 28.38 & $* * *$ \\
\hline 15 & loss of libido & 27.5 & 18.6 & 2.56 & \\
\hline 16 & solve a mental task & 25.0 & 20.4 & 0.68 & \\
\hline 17 & difficulty concentrating & 25.0 & 22.2 & 0.25 & \\
\hline 18 & shrink from work & 26.3 & 8.4 & 14.20 & 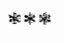 \\
\hline 19 & dead end & 25.0 & 4.8 & 21.98 & $* * * *$ \\
\hline 20 & crying & 12.5 & 9.6 & 0.49 & \\
\hline 21 & defeated & 20.0 & 4.8 & 14.26 & $* * * *$ \\
\hline 22 & hopelessness & 22.5 & 7.2 & 11.89 & *** \\
\hline 23 & death wish & 10.0 & 3.0 & 5.32 & * \\
\hline
\end{tabular}

$* p<0.05 ; * *<<0.01 ; *$ ***; $p<0.001$

Forty cases $(50 \%)$ endorsed eight items or more. The average duration of feelings of VE was 3 months in $13 \%, 6$ months in $30 \%$, one year in $27 \%$ and 18 months in $10 \%$. Twenty percent of the patients had experienced exhaustion for over 18 months. Twenty-seven controls $(16 \%)$ endorsed eight items or more. The percentages of the average duration of the feelings of VE were $0 \%$ three months, $11 \% 6$ months, $30 \%$ one year, $11 \%$ eighteen months and $48 \%$ more than 18 months. Applying the decision rule to label those whose complaints had lasted longer than 18 months as non- 
exhausted, $40 \%$ of the cases and $8 \%$ of the controls were exhausted before myocardial infarction or before the interview.

\subsection{CORRESPONDENCE BETWEEN THE INTERVIEW AND THE QUESTIONNAIRE}

To investigate the correspondence between the questionnaire and the interview we computed the product-moment correlation between the scores on these measures and the percentages of subjects who are classified as exhausted or non-exhausted by the questionnaire as well as by the interview. Subjects with MQ-scores one standard deviation above the mean of the reference group of the RCSS (Appels \& Mulder, 1988) were labeled "exhausted".

Table 6.2. Correspondence between the Maastricht Interview for Vital Exhaustion (MIVE) and the Maastricht Questionnaire (MQ) for cases and controlls

\begin{tabular}{|c|c|c|c|}
\hline & & \multicolumn{2}{|c|}{ MIVE } \\
\hline & & $\begin{array}{c}\text { exhausted } \\
\text { (MIVE } \geq 8 \text { ) }\end{array}$ & $\begin{array}{c}\text { non-exhausted } \\
\text { (MIVE } \leq 7 \text { ) }\end{array}$ \\
\hline \multirow{6}{*}{$\mathrm{MC}$} & CASES & & \\
\hline & exhlhausted (MQ $\geq 17)$ & 26 & 10 \\
\hline & non-exhausted (MQ $\leq 16)$ & 6 & 38 \\
\hline & CONTROLS & & \\
\hline & exhausted (MQ $\geq 17$ ) & 10 & 34 \\
\hline & non-exhausted (MQ $\leq 16$ ) & 4 & 1.19 \\
\hline
\end{tabular}

As expected, interview scores correlated strongly with questionnaire scores, the correlation coefficient being 0.74 . Table 6.2 shows the correspondence between the interview and the questionnaire as to classification of subjects as exhausted or nonexhausted. The table shows that 36 out of the 80 cases $(45 \%)$ are classified as exhausted by the questionnaire and $32(40 \%)$ by the interview. Out of the 167 controls 44 subjects $(26 \%)$ are classified as exhausted by the questionnaire and $14(8 \%)$ by the interview. Only 10 out of the 44 controls $(23 \%)$ who were exhausted according to the MQ were also classified as exhausted by the interview. These results indicate that more subjects are classified as exhausted by the questionnaire than by the interview. 


\subsection{DISCRIMINATING POWER OF THE QUESTIONNAIRE AND THE INTERVIEW}

The discriminating power of the interview and the questionnaire was investigated by means of a $t$-test and a multiple logistic regression analysis, controlling for age as a possible confounder. Table 6.3 shows the mean scores of cases and controls on both instruments. As is indicated by the magnitude of the t-values, the interview discriminates better between cases and controls.

The results of the logistic regression analyses showed that the age-adjusted odds ratios of the interview and the questionnaire are 9.50 and 2.26 , respectively. Standardization of these odds ratios, to make them directly comparable, demonstrated that the interview discriminated twice as well as the questionnaire, the Standardized Odds Ratios (SORs) being 0.44 and 0.23 respectively. These statistics clearly indicate that, compared to the MQ, the interview has more discriminating power.

Table 6.3. Mean scores on the Maastricht Interview for Vital Exhaustion (MIVE) and the Maastricht Questionnaire (MQ) comparing cases and controls by means of t-test

\begin{tabular}{lccccc}
\hline & N & Mean & s.d. & t & p(two-tailed) \\
\hline $\begin{array}{l}\text { MIVE } \\
\text { casses }\end{array}$ & 80 & 7.8 & 6.0 & & \\
controls & 167 & 3.6 & 4.0 & 5.65 & 0.001 \\
$\begin{array}{l}\text { MQ } \\
\text { cases }\end{array}$ & 81 & 1.5 .2 & 11.7 & & \\
controls & 168 & 11.8 & 10.5 & 2.31 & 0.022 \\
\hline
\end{tabular}

Note: Small differences in $N$ are due to missing data.

\subsection{DISCUSSION}

The aim of this article is to describe the development and content of an interview to assess vital exhaustion, a state which has been found to be a short-term risk indicator of myocardial infarction. The need to develop an interview emerged in studies which used the MQ to select exhausted and non-exhausted subjects for experimental studies. These studies suggested that elevated scores are not always indicative of a state of vital exhaustion.

In the absence of a gold standard against which the validity of the questionnaire and the interview can be compared we chose their discriminating power with regard to discrimination between cases and controls as the external criterion. The results of this comparison clearly indicated that the interview has more discriminating power than the 
questionnaire. If the interview is taken as the standard and the control group as representative of the population, it can be said that the extent to which the MQ yields results free from false positives is $23 \%(10 / 44)$. This statistic confirms the earlier observation that elevated questionnaire scores are not necessarily indicative of a state of VE. In contrast, the extent to which the MQ gives results free from false negatives is excellent $(97 \% ; 119 / 123)$.

The aim of this study was the development of an interview to assess vital exhaustjon. The interview might both be used as a prognostic test in cardiovascular research and as a diagnostic test for the selection of exhausted subjects for experimental studies, which, for example, address the biological mechanisms underlying the association between vital exhaustion and myocardial infarction. Given the relatively large number of false positives produced by the $\mathrm{MQ}$ we recommend to use the interview for the selection of exhausted subjects for small-scale studies.

There are several reasons why the comparison of the questionnaire and the interview should be interpreted cautiously. The discriminating power of the interview may have been overestimated because the interviewers were aware of the cardiac status of the subjects interviewed, which was unavoidable in the present study. Furthermore, although the decision rules to place the cut-off score between 7 and 8 and to classify those who had experienced feelings of exhaustion for more than 18 months as nonexhausted are not arbitrary, they remain open to cliscussion.

It is beyond dispute that each interview method has its own sources of bias which may influence the reliability and validity of the assessment. The style of interviewing and difficulties in scoring ambiguous answers make the final score interwiewerdependent and therefore less objective. For this reason, we have added detailed instructions on how the interview should be administered and scored so as to reduce the subjectivity of the interview. Reliability and validity of the interview will be discussed in the companion paper (Meesters \& Appels, 1995b). 


\section{APPENDIX I}

\section{THE MAASTRICHT INTERVIEW FOR VITAL EXHAUSTION (MIVE)}

Respondent

Name.

Date.

Interviewer.

1. Do you often feel tired?

If so, for how long have you been feeling tired?

2. Have you felt listless lately?

If so, for how long have you been feeling listless?

3. Do you feel weak all over or without energy?

If so, for how long have you been feeling weak?

4. Have you been irritated more easily lately than before?

$+-?$

If so, when did this start?

5. Do you feel dejected?

What makes you feel that way?

6. Do you sometimes feel as if your body is like a battery that is losing its power?

7. Do you have the feeling that you have not been accomplishing much lately or that you are less capable of accomplishing things?

$+\quad-$ ?

8. Do you ever wake up with a feeling of exhaustion or fatigue?

$+\quad-?$

9. Do you wake up repeatedly during the night? If so, do you consider this a problem?

10. Do you have the feeling that you cannot cope with everyday problems as well as you used to?

11. Do you feel you want to give up trying?

12. Have little things imitated you mowe lately than they used to? 
13. Do you blow up more easily than before?

14. Do you have the feeling these days that you just do not have what it takes anymore?

15. Have you noticed a decrease in your sexual appetite or a decrease in the desire to make love?

16. Has it become harder lately to solve a mental task or problem which requires much concentration?

17. Do you have increasing difficulty in concentrating on a single subject for long?

18. Do you shrink from your regular work as if it were a mountain to climb?

19. Do you believe that you have come to a "dead end"?

$+\quad-?$

20. Do you sometimes cry or feel like crying?

21. Do you feel defeated or disillusioned?

22. Have you experienced a feeling of hopelessness recently?

$+-?$

23. Would you want to be dead at times?

24. If applicable:

During our conversation you have described yourself as.......

I understood that this state started .... months/years ago.

Is this correct?

Interviewer: now check whether all questions have been asked and coded.

25. Interview score vital exhaustion 


\section{APPENDIX II}

\section{ADMINISTERING AND SCORING THE INTERVIEW}

\section{GENERAL REMARKS}

1. The best preparation for proper assessment of the interview is to interview a number of coronary patients to learn to recognize some qualitative characteristics of exhaustion. MI patients who were exhausted before the event will immediately recognize what the question is about. They will, for example, never ask, "What do you mean by waking up exhausted?" and rarely giwe an answer hesitantly.

2. The interview questions are phrased in the present tense. The present tense is to be used for the selection of exhausted subjects for small-scalle studies. Those who use the interview for coronary patients to assess prodromal feelings of MI should rephrase each question in the past tense. Retrospective information may be biased by a search for meaning or by an expectancy bias (the interviewer is supposed to be interested in stress symptoms). To prevent these biases from occurring, a retrospective interview may start. with the following instruction, "MI is still a mystery in many respects. Some people have the feeling that $M I$ is foreshadowed by certain warning signals. Other people do not believe so. We just do not know. You can help us find out by answering some questions".

3. The interview asks about the present state. Subjects may sometimes answer "Yes, I was..., but nowadays I am...". Such answers must be scored as negative. Note that questions $4,12,13$, and 17 explicitly ask about increases in at specific behaviour. In case the response to these four questions is, "Yes, I have always been like that" the answer should be scored as negative.

4. Answers like "sometimes", "some" or "somewhat" are usually not indicative of VE. In case of doubt, the interviewer may repeat a question at the end of the interview. He may also ask, "If you had to answer either "Yes" or "No" to this question, what would you say?"

5. If the exhaustion has lasted for more than 18 months a subject should not be classified as exhausted, because VE is not a chronic condition. We recommend exclusion of such a person from a laboratory study.

6. Further research is needed to gain a deeper insight into the correspondence between exhaustion and depression. What can be said, however, is that loss of self-esteem, feelings of guilt, and mood disturbances (feeling sad or blue) are usually absent in exhausted subjects. 
Depressed patients often feel tired, but exhausted subjects are not necessarily depressed. Elevated scores may be observed among those who are recovering from depression. We recommend exclusion of this group if the interview is used to recruit participants in a laboratory study.

7. In case the interview is used to select a relatively small number of exhausted and nonexhausted subjects for a laboratory study we recommend using the dichotomous classification and exclusion of those who did not endorse two of the following items: $1,2,3$, $6,8,11,14$, and 18 .

\section{SPECIFIC INSTRUCTIONS}

item 1 (tired)

Some respondents (especially unemployed respondents) may say that the tiredness is due to the absence of a need to mobilize energy. If so, the interviewer should add, "Do you believe that you would feel less tired if you could would work again?" If the subject gives a positive answer to the latter question item 1 should be scored as negative.

item 2 (listless)

Answers like "I have no go" or "I have no grit" must be scored as positive.

item 3 (lack of energy)

Some respondents mention a decrease in energy after they have stopped practising sports. This does not reflect an inability to summon up energy.

item 4 (irritability)

Some coronary patients attribute the increased irritability to the debilitating effects of angina pectoris. If so, the item must be scored as positive.

Some respondents answer, "You should ask others about this". If so, add the question, "What would they say?" or "Did somebody (your wife, children, colleague) tell you that you were easily irritated?" If others have noticed an increased irritability the item is scored as positive.

item 6 (empty battery)

MI patients often say that this is the best description of how they felt before their cardiac event. During the interview a subject may use other metaphors to describe the exhaustion such as "the well is drying up" or "the balloon is empty". These idiosyncratic descriptions provide valid information. In case a subject uses a different metaphor during the interview to describe the depletion of energy, item 6 must be scored as positive, even if the answer to question 6 is negative.

Many subjects may answer, "Yes, I am growing older, I am no longer in my twenties". If so, the interviewer may add, "Do you believe that your battery has less power compared to other persons your age?" or "Suppose that we were talking about the battery of your car, would the warning signal on the dashboard be on?" 
item 7 (inability to accomplish things)

This item registers an inability to summon up energy. A positive answer which is attributed to age might be a false positive. Add the question. "Are you less able to achieve things" than the average person your age?"

Subjects who have stopped working may answer, "Yes, because there is no need to accomplish anything anymore". If this answer is given, the following question should be added: "Do you believe that you could accomplish things if you were still working?"

item 8 (waking up exhausted)

Answers like "I have the feeling that I did not sleep long enough" are not indicative of exhaustion.

item 9 (disrupted sleep)

Waking up to urinate followed by a continuation of sleep is not indicative of a sleep problem.

The answer is scored as positive only in case the disrupted sleep is experienced as a problem.

item 10 (inability to cope with everyday problems)

If" a subject finds it hard to answer, the question can be rephrased as, "Do you feel that you are overburdened?"

item 11 (give up trying)

In case the subject is unemployed, the item may be rephrased as, "Do you want to stop your hobbies, social activities?"

In some subjects, the question evokes suicidal ideation. If so, the answer must be scored as positive.

item 14 (decrease in energy)

This item asks about a decrease in energy to start an activity. Unemployed or retired subjects may stress the absence of stimuli to become active. If so, ask whether the subject finds it difficult to summon up the energy to do things at home or to participate in social activities.

item 16 (solve a mental task)

This item asks about a decrease in cognitive functioning. It may be helpful to give examples like reading a newspaper, selecting the right lane at a crossroads, writing a report. Positive answers include, "It took more time", "I made more mistakes", "I had to check more often".

item 18 (shrink from work)

In case the subject is unemployed or retired the question may be rephrased as, "Do you shrink from doing things like shopping, gardening or driving a car?"

item 19 (dead end)

In case the subject has difficulty undersianding the question it may be rephrased as, "Do you have the feeling that you are in a situation with no way out?" 
item 20 (crying)

In a state of exhaustion, a subject may be hyperaesthetic or easily emotionally affected. A positive answer might be e.g. "I stopped watching television because I could not stand the misery they show anymore."

itern 23 (death wish)

A positive answer may reflect a wish to be dead or very strong annoyance. In both cases the answer must be scored as positive.

item 24 (summary)

This item provides the opportunity for the total picture to be verified by the respondent and by the interviewer. It also provides an opportunity to check whether all questions have been askedi and to ask questions coded as "?" again. 


\section{AN INTERVIEW TO MEASURE VITAL EXHAUSTION. II. RELIABILITY AND VALIDITY OF THE INTERVIEW AND CORRELATIONS OF VITAL EXHAUSTION WITH PERSONALITY CHARACTERISTICS ${ }^{1}$}

\subsection{SUMMARY}

In the previous paper (Meesters \& Appels, 1995a) we described the development of an interview to measure feelings of vital exhaustion (VE). The current paper presents data on the reliability and the validity of the interview, which is labeled the Maastricht Interview for Vital Exhaustion (MIVE). Correlations with the Type A behavior pattern (TABP) and hostility are also discussed in this paper. The data were collected in a case-control study of 81 males with first MI and 168 healthy neighborhood controls. The interview was found to have a good reliability, Cronbach's $\alpha$ being 0.90 in the case group and 0.86 in the control group. The kappa coefficient was 0.95 in the case group and 0.91 in the control group. The validity was tested by computing the estimated relative risk of $\mathrm{VE}$, controlling for angina pectoris and the classic risk factors. The odds ratio was found to be $8.11(\mathrm{p}<0.001)$. This indicates that the interview makes a valid assessment of the mental precursors of MI. The interview correlated significantly with TABP (structured interview) and two self-report measures of hostility (Cook-Medley Hostility Scale and Buss-Durkee Hostility Inventory). Although the absolute magnitude of the observed odds ratios may be affected to some extent because the interviews were not scored blind, these results clearly suggest that the MVE is a reliable and valid method to assess VE.

\subsection{INTRODUCTION}

Feelings of excess fatigue and loss of energy have been amply reported in the literature as the most frequent precursors of myocardial infarction (MI) and sudden death (SD). We have labeled these feelings vital exhaustion (VE) and defined VE as a state characterized by unusual fatigue and loss of energy, increased irritability and feelings

\footnotetext{
'Meesters, C., \& Appels, A. (1995). An interview to measure vital exhaustion. II. Rellability and validity of the interview and correlations of vital exhaustion with personality characteristics. Psychology and Health, in press.
} 
of demoralization. Two prospective and several case-control studies have shown that this state precedes the onset of new cardiac events (for a thorough review see Appels et al., 1994). In these sudies VE was measured by means of the Maastricht Questionnaire (MQ). Although this instrument has good psychometric qualities "it was observed that its use as a screening instrument for the selection of subjects for smallscale or experimental studies was limited due to a significant number of false positives (van Diest \& Appels, 1994). To overcome this limitation, we designed a structured interview to measure VE. The theoretical background of this interview, its development and the way it is scored was described in detail in the previous paper (Meesters \& Appels, 1995a).

In the current paper the following questions will be addressed. What is the reliability and the validity of the interview and what is the correlation between interview scores and some selected personality characteristics? The reliability will be investigated by computing the internal consistency and the correspondence between the interview scores of two independent observers. The latter is important because interviews are vulnerable to subjective elements in the administering of the interview and in the interpretation of the answers. In the absence of a gold standard to assess VE, the ability of the interview to identify subjects at elevated risk of myocardial infarction was chosen as the validity criterion. The interview will be considered to be valid if the estimated relative risk of VE is significantly larger than 1.00 after controlling for angina pectoris and the classic cardiovascular risk factors. Finally, we will present the correlations between VE and certain personality characteristics. Because this study was made in a cardiovascular context, two personality characteristics which are supposed to increase the risk of MI were selected, viz. TABP and hostility (Siegman, 1994a). The data needed to answer the three questions mentioned above were collected in a case-control study.

\subsection{METHOD}

\section{Subjects and procedure}

The subjects were 81 males who were hospitalized because of a first clinically documented MI and 168 age-matched neighborhood controls. Cases were asked how they had felt in the months before hospitalization, while controls were asked how they had felt during the last few months. The mean age of the cases was 55.6 (s.d. 6.8) years. The mean age of the controls was 54.9 (s.d. 6.9) years. Cases and controls who were willing to participate received a set of questionnalres and were later interviewed at home. The previous paper presents a detailed description of subjects and procedures (Meesters \& Appels, 1995a). 


\section{Instruments}

\section{Questionnaires}

Both cases and controls completed the following questionnaires:

The retrospective version of Form B of the Maastricht Questionnaire (MQ), which is a 21 -item checklist to assess manifestation of vital exhaustion during the last six months (prior to MI) (Appels, Höppener, \& Mulder, 1987)

The Dutch version of the Cook and Medley (1954) (Ho) Scale, a 50-item true-false paper-and-pencill test measuring a general index of cynical mistrust (Williams \& Barefoot, 1988).

The Dutch version of the Buss-Durkee Hostility Inventory (BDHI; Buss \& Durkee, 1957), consisting of 75 items in a true-?-false format. Seven types of hostility are included: Assault, Indirect, Verbal, Irritability, Negativism, Resentment, and Suspicion. The scale also has a subscale to measure feelings of guilt.

The London School of Hygiene Cardiovascular Questionnaire for the assessment of angina pectoris within the last six months (prior to MI) (Rose, Blackburn, Gillum \& Prineas, 1982).

A questionnaire asking questions about demographic characteristics and cardiovascular risk factors. The questions concern: marital status, educational level, smoking, coffee consumption, high blood pressure, diabetes mellitus, and family history of $C H D$.

\section{Interviews}

About four weeks after discharge from hospital, MI-cases were interviewed at home. Neighborhood controls (NCs) were also interviewed at home, roughly four weeks after the questionnaires had been mailed. Two interviews were administered: the Maastrich Interview for Vital Exhaustion (MIVE) (Meesters \& Appels, 1995a) to assess VE and the Structured Interview (SI) (Rosenman, 1978) for the assessment of global Type A Behavior Pattern (TABP). Ml-cases were asked how they had relt before hospitalization, while NCs were asked how they had felt during the last month. Both interviews were taperecorded. The exhaustion interviews were scored independently by the two authors. All Type A interviews were scored independently by two blind raters afterwards.

\section{Data analysis}

Data analysis started with a factor analysis of the interview to examine whether the interview has one or more dimensions. An iterative principal factor analysis of all interview items and all subjects was used. Cronbach's $\alpha$ was calculated for the case group and for the control group to establish the homogeneity or the internal consis- 
tency of the interview. In order to assess interrater agreement kappa statistics were computed for both cases and controls.

Investigation of the validity of the interview started with computation of the association between interview scores, on the one hand, and angina pectoris and the cardiovascular risk factors age, smoking, high blood pressure, diabetes mellitus, and family history of CHD, on the other hand. Smoking was coded as non-smoking (never smoked and stopped smoking), moderate smoking (1-19 cigarettes daily), and heavy smoking ( 20 cigarettes or more daily) during the last six months before hospitalization or the interview. Hypertension was defined as having been treated for hypertension during the six months preceding hospitalization or the interview, diabetes as receiwing treatment for diabetes. A positive family history of CHD was defined as having a parent or sibling who had suffered from a fatal or non-fatal myocardial infarction at some time. The associations between these risk factors and VE were investigated by means of univariate t-tests and one-way analyses of variance (ANOVA).

The discriminating power of the interview was tested by multiple logistic regression analyses, using SPSS/PC 4.0 (Norusis, 1990). Patient status (MI or NC) served as the dependent variable, the controls serving as the reference group. Vital exhaustion was included in the model as a categorical variable. Subjects with an interview score equal to or greater than 8 were classified as "vitally exhausted" if the complaints had lasted no longer than 18 months. Cases and controls whose complaints had lasted for more than 18 months were classified as non-exhausted. Age was included as a continuous variable. The first step consisted in computation of the age-adjusted odds ratio. An interaction between VE and age was included in this analysis, because VE was found to be differentially associated with age in cases and controls. In the next step, angina pectoris and the classic risk factors were included in the model. Both direct and stepwise procedures with maximum likelihood testing were used to obtain the most parsimonious model.

The relationship between vital exhaustion and TABP and hostility was explored using univariate t-tests and product-moment correlations, respectively.

Due to missing data there may be small differences in the number of subjects in several tables.

\section{$7.4 \quad$ RESULTS}

\section{Reliability}

The results of the principal factor analysis are summarized in table 7.1. One conceptually clear factor explaining $33 \%$ of the total variance emerged, with all 23 items loading on the factor. Four items had factor loadings $<0.40$. Three of these four items did not discriminate significantly between cases and controls (see the first paper; Meesters \& Appels, 1995a). Although these items do not contribute to the 
reliability and validity of the interview we decided not to remove them, matryly because they were predictive of MI in the earlier prospective study (Appers Mul. der, 1988).

Table 7.1. Factor loadings of the items of the Maastricht Interview for Vital Exhaustion

\begin{tabular}{llc}
\hline item & content & loading \\
\hline 01 & tired & 0.58 \\
02 & listless & 0.76 \\
03 & lack of energy & 0.68 \\
04 & irritability & 0.57 \\
05 & dejected & 0.63 \\
06 & empty battery & 0.61 \\
07 & inability to accomplish & 0.61 \\
08 & waking up exhausted & 0.65 \\
09 & disrupted sleep & 0.39 \\
10 & inability to cope & 0.67 \\
11 & give up trying & 0.49 \\
12 & annoyed & 0.50 \\
13 & blow up & 0.54 \\
14 & decrease in energy & 0.64 \\
15 & loss of libido & 0.33 \\
16 & solve a mental task & 0.31 \\
17 & difficulty concentrating & 0.48 \\
18 & shrink from work & 0.50 \\
19 & dead end & 0.66 \\
20 & crying & 0.38 \\
21 & defeated & 0.51 \\
22 & hopelessness & 0.49 \\
23 & death wish & 0.47 \\
eigenvalue factor: 7.54 & (before rotation) & \\
explained variance: $33 \%$ & ing & \\
\hline & m & \\
\hline
\end{tabular}

Cronbach's $\alpha$ was found to be 0.90 in the case group and 0.86 in the control group. These statistics indicate that the interview measures an internally consistent construct. The kappa statistic (Cohen, 1960) for interrater agreement was 0.95 in the case group and 0.91 in the control group, which indicates a high interrater agreement.

\section{Validity}

Association with CHD risk factors

Scores on the MIVE were found to be negatively associated with age $(r=-0.21$, $p<0.01)$. In particular in cases a strong negative association was observed $(r=-0.41$, $\mathrm{p}<0.001$ ). In controls the product-moment correlation is -0.14 (n.s.). 
Table 7.2. Association between vital exhaustion as assessed by the MIVE and risk factors of CHD

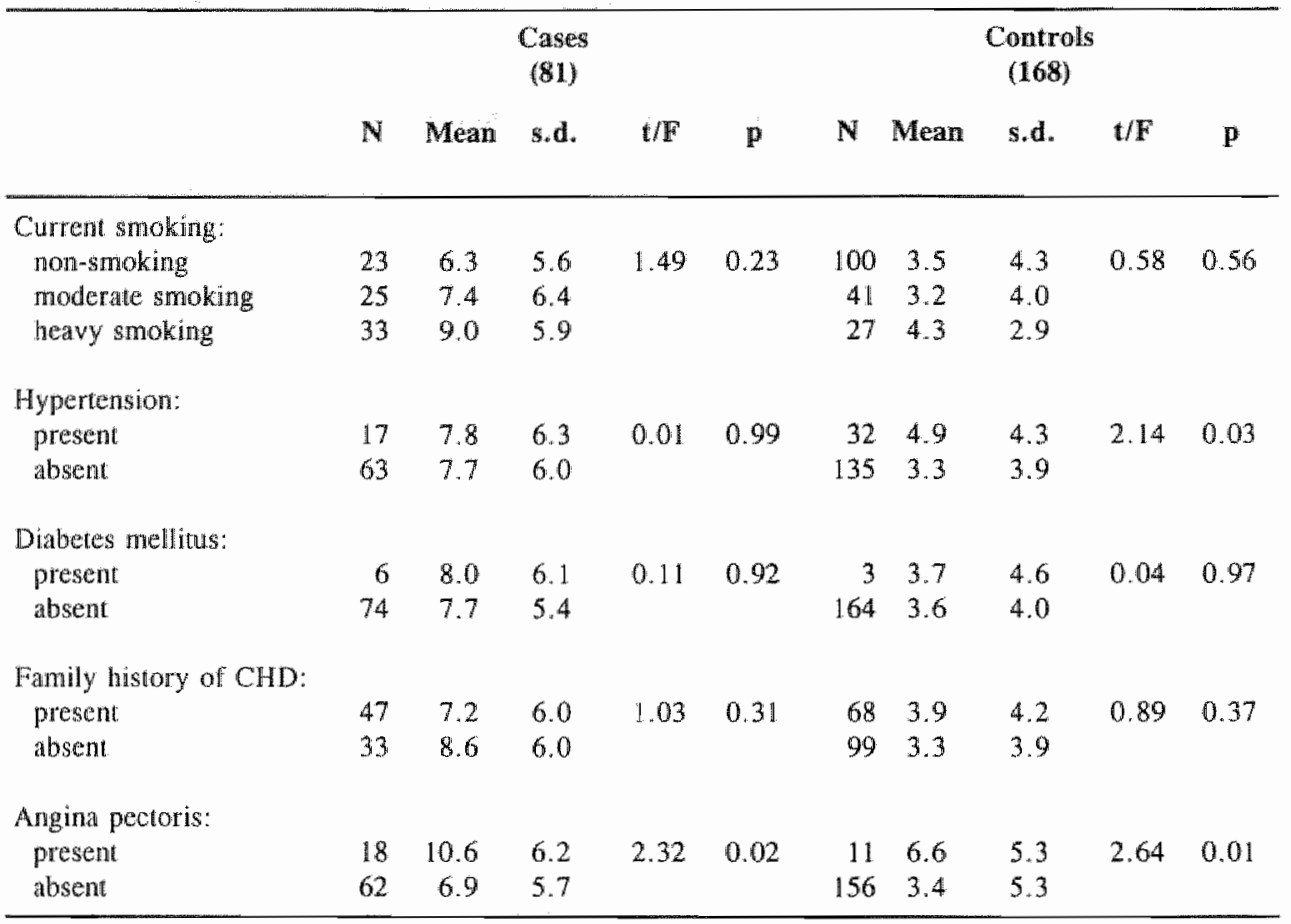

As is shown in table 7.2, heavy smokers tend to have higher scores on the MIVE. However, smoking was not significantly associated with interview scores, neither in cases nor in controls. In the case group mean scores on the interview did not differ between hypertensive and non-hypertensive subjects, but in the control group a positive relationship was observed between this sel-report index of elevated blood pressure and vital exhaustion. Control subjects who had received treatment for elevated blood pressure during the past six months had significantly higher interview scores than non-hypertensive controls. No association between vital exhaustion and diabetes and a positive family history was observed.

Twenty-nine subjects (18 cases and 11 controls) suffered from angina pectoris. In the case group as well as in the control group subjects with anginal complaints reported significantly higher levels of vital exhaustion than subjects free from angina. Particularly MI cases suffering from angina showed elevated scores on the interview.

\section{Validity of the interview}

The first logistic regression analysis showed that there was no significant interaction between age and VE upon the risk of MI. Therefore, the interaction term was omitted in all subsequent analyses. Forward and backward procedures resulted in the 
same parameter estimates. In all models, hypertension and diabetes were removed from the final model, which is presented in table 7.3. The table shows that the risk of vital exhaustion as assessed by the interview, controlling for angina pectoris and the classic risk factors, is 8.11 . These findings show that the interview is able to identify subjects at elevated risk of myocardial infarction. Therefore, the interview may be considered to be a valid indicator of this prodromal state.

Table 7.3. Maximum likelihood estimates of logistic parameters relating vital exhaustion as assessed by the MIVE, age, smoking, angina pectoris, and family history of CHD to the risk of myocardial infarction

\begin{tabular}{lcccc}
\hline variable & $\beta^{\mathbf{x}}$ & $\mathbf{s e}^{2}$ & $\mathrm{OR}^{3}$ & $\mathbf{p}$ \\
\hline Vital exhaustion & 2.09 & 0.43 & 8.11 & 0.00 \\
Age & 0.06 & 0.02 & 1.06 & 0.00 \\
Smoking & & & & \\
$\quad$ moderate smoking & 0.98 & 0.39 & 2.67 & 0.01 \\
$\quad$ heavy smoking & 1.59 & 0.40 & 4.91 & 0.00 \\
Angina pectoris & 1.23 & 0.48 & 3.43 & 0.01 \\
Farmily history of CHD & 0.98 & 0.33 & 2.66 & 0.00 \\
\hline
\end{tabular}

Logistic regression coefficients

2 standard errors

${ }^{3}$ odds rarios

\section{Relationship with TABP and hostility}

Interrater agreement on the Type A interview was $82 \%$, which corresponds to proportions reported elsewhere (e.g. Caffrey, 1968; Jenkins, Rosenman, \& Friedman, 1968; Keith, Lown, \& Stare, 1965; Scherwitz, Berton, \& Leventhal, 1977).

Table 7.4 shows the association between TABP and vital exhaustion. Type A subjects report higher levels of vital exhaustion than Type B subjects, especially in the MI case group. VE also proved to be positively associated with scores on several dimensions of hostility (see table 7.5).

Table 7.4. Mean scores on the Maastricht Interview for Vital Exhaustion (MIVE) for cases and controls comparing Type As and Type Bs by means of t-test

\begin{tabular}{lcccccc}
\hline \multirow{2}{*}{ Cases } & & N & Mean & s.d. & t & p \\
\hline \multirow{4}{*}{ Controls } & Type A & 49 & 9.7 & 5.7 & & \\
& Type B & 31 & 4.6 & 5.1 & 4.03 & 0.000 \\
& Type A & 94 & 3.9 & 4.3 & & \\
& Type B & 73 & 3.1 & 3.7 & & 0.22 \\
\hline
\end{tabular}


Table 7.5. Product-moment correlations of the Maastricht Interview for Vital Exhaustion (MIVE) with the $\mathrm{Ho}^{\mathrm{I}}$ scale and $\mathrm{BDHO}^{2}$ subscales

\begin{tabular}{lll}
\hline & $\begin{array}{l}\text { Cases } \\
(\mathbf{n}=80)\end{array}$ & $\begin{array}{c}\text { Controls } \\
(\mathbf{n}=\mathbf{1 6 6})\end{array}$ \\
\hline Ho & $0.24 *$ & $0.33^{* * *}$ \\
BDHI Total & $0.42^{* * *}$ & $0.25^{* * *}$ \\
Assaul & $0.44^{* * *}$ & $0.18^{*}$ \\
Indirect Hostility & $0.37^{* * *}$ & 0.13 \\
Irritability & $0.41^{* * *}$ & $0.30^{* * *}$ \\
Negativism & 0.12 & 0.06 \\
Resentment & $0.37^{* * *}$ & $0.39 * * *$ \\
Suspicion & 0.08 & $0.17^{*}$ \\
Verbal Hostility & 0.17 & -0.01 \\
Guilt & 0.05 & $0.23 * *$ \\
\hline
\end{tabular}

Cook-Medley Hostility Scale

${ }^{2}$ Buss-Durkee Hostility Inventory

* $p<0.05 ; * * p<0.01 ; * * * p<0.001$

\subsection{DISCUSSION}

The main aim of this study was to test the reliability and validity of vital exhaustion, as assessed by a structured interview. It was shown that the reliability of the interview is good, which becomes clear from the observed Cronbach's $\alpha$ 's and the high interrater agreement. The risk of a state of exhaustion for suffering from myocardial infarction was highly significant. This confirms its validity as an instrument to measure prodromal feelings of $\mathrm{MI}$.

The results also show that vital exhaustion is associated with TABP. Type A subjects exhibit higher levels of exhaustion than Type B subjects. This relationship was also reported by Falger (1989). Vital exhaustion also proved to be positively associated with scores on several dimensions of hostility. These findings suggest that Type A subjects and hostile persons are at risk to exhaust themselves.

Subjects suffering from angina pectoris were found to have elevated interview scores. This may indicate that vital exhaustion increases the risk of angina pectoris. This interpretation is in line with the findings of the prospective Rotterdam Civil Servants Study (RCSS) (Appels \& Mulder, 1988). However, angina pectoris may also cause feelings of exhaustion. Therefore, angina pectoris might be considered as a confounder of the association between vital exhaustion and MI. The decision to include angina pectoris in the equation may have resulted in an underestimation of the odds ratio. 
Age was found to be negatively associated with vital exhaustion, in particular in cases. A number of patients (and some of their physicians) are inclined to attribute the feelings of tiredness and loss of energy to ageing. The rather strong negative correlation coefficients show that this interpretation may be incorrect. In the control group, a positive relationship was found between high blood pressure and vital exhaustion. Hypertensive controls reported significantly higher levels of vital exhaustion than non-hypertensive controls. This may either be indicative of a side effect of drugs or of a higher detection rate of hypertension among those who are exhausted.

There are several reasons why the data concerning reliability and validity should be interpreted cautiously. First of all, the interviewers were aware of the cardiac status of the subjects, which was unavoidable because of the retrospective nature of the study and the set-up of the interview. Scoring of the interview by independent raters does not solve this problem, because many patients refer to their disease during the interview. An interview is not onlly vulnerable to subjective elements in the scoring of the answers, but also to differences in interview style. In this study, nearly all interviews were carried out by the first author. This may have resulted in suppression of interviewer bias and thus to an overestimation of the interrater agreement.

The absolute magnitude of the observed odds ratios might be biased for several reasons. As was said before, it was impossible to score the interviews blind. This may have affected the difference between cases and controls to an unknown degree. However, we believe that the scoring was not strongly affected by knowledge of the disease status, because all questions are quite straightforward. Furthermore, in case of doubt a symptom was rigorously scored as being absent in order to avoid ambivalent statements being scored as positive in cases and negative in controls.

Secondly, the absolute magnitude of the odds ratios may also have been overestimated because it was impossible to control for subclinical heart disease. We had to rely upon a non-clinical method to assess angina pectoris and information about the left ventricular ejection fraction was not available. However, we do not believe that the odds ratios have been seriously overestimated because we could not control for subclinical heart disease. Kop has demonstrated that vital exhaustion is not associated with left ventricular ejection fraction and only marginally with the extent of coronary atherosclerosis (Kop, 1994 ).

Thirdly, the absolute magnitude of the odds ratios may also have been overestimated by the retrospective nature of the study. Cases were asked how they felt during the months before hospitalization. Their answers may have been influenced by a search for meaning and by the disease itself. However, we are inclined to believe that the data are not strongly influenced by a retrospective bias. Usually patients make a clear distinction between their present state and how they felt before the occurrence of the cardiac event. Most importantly, the estimated relative risk of 8.11 corresponds well with the relative risk of $\mathrm{MI}$ in the first year of follow-up (10.01) observed in the prospective study (Appels \& Mulder, 1988). However, the strength of the association is sufficient to cause some concern. It is possible that vital exhaustion does not only reflect a breakdown in adaptation to stress, but is also a marker of 
subclinical heart disease which is not reflected by anginal complaints, impaired left ventricular ejection fraction or the extent of atherosclerosis. However, it is still unknown which elements of the pathophysiology of the acute coronary syndromes may cause feelings of VE.

An important question remains whether there is a distinction between vital exhaustion and depression. There is accumulating evidence that depression is a risk factor with regard to first and recurrent MI (Carney et al., 1988; Ladwig, Kieser, Konog, Breithardt, \& Borggrefe, 1991; Silverstone, 1987). Is the existence of vital exhaustion as a concept separate from depression warranted? So far, we think it is. Vital exhaustion shares a substantial number of symptoms with clinical depression as defined by the DSM-IV (APA, 1994). However, mood disturbances are usually absent in exhausted subjects (van Diest \& Appels, 1991), and so are the core symptoms of depression described by cognitive psychology ("I am the cause of my misfortune"; "I always will be a failure"; "Whatever I do will be a failure"). Self-esteem is rarely lowered in coronary patients. Therefore, it is our opinion that most depressives are exhausted and that only a minority of exhausted subjects are depressed in a clinical sense. 


\section{CHAPTER 8}

\section{HOSTILITY, VITAL EXHAUSTION AND MYOCARDIAL INFARCTION: DISCRIMINATING POWER AND INTERRELATIONSHIPS ${ }^{1}$}

\subsection{SUMMARY}

Several studies have indicated that both hostility and vital exhaustion may increase the risk of myocardial infarction (MI). However, their interrelationship has never been investigated. To test the hypothesis that hostility contributes to the development of a state of exhaustion preceding MI, both factors were measured in a case-control study. Subjects were 81 male patients with a first MI (mean age 55.6 years; SD 6.8) and 168 male neighborhood controls (mean age 54.9 years; SD 6.9). Hostility was measured by means of the Cook-Medley Hostility ( $\mathrm{Ho}_{0}$ Scale and the Buss-Durkee Hostility Inventory (BDHI). VE was assessed by means of the Maastricht Interview for Vital Exhaustion (MIVE). Vital exhaustion was found to be positively related to several dimensions of hostility. Separate multivariate analyses for each of the hostility scales and for vital exhaustion revealed that vital exhaustion and the experiential hostillity factor of the BDHI were both significantly associated with MI, while adjusting for age, smoking, angina pectoris, hypertension, diabetes mellitus, and family history of CHD. No significant relationship was observed between any other hostility measures and $\mathrm{MI}$.

When hostility, VE, and MI were all included in the same logistic model, in order to test their association, all hostility dimensions lost their discriminating power.

These findings corroborate earlier observations that both hostility (especially as operationalized by the BDHI) and vital exhaustion increase the risk of MI. They further indicate that the risk of hostility may be due to the fact that hostile persons are at elevated risk of becoming exhausted.

\subsection{INTRODUCTION}

In the past decade, both hostility and vital exhaustion have been found to be risk indicators of coronary heart disease (CHD). Following the Type A behavior pattern

'Meesters, C., \& Appels, A. (1995). Hostility, vital exhaustion and myocardial infarction: Discriminating power and interrelationships. (submitted) 
(TABP) research initiated by Friedman and Rosenman in the late $1950 \mathrm{~s}$, a large number of studies linking hostillity to adverse health outcomes (especially cardiovascular disease) have now been published. Hostility is considered a relatively enduring personality trait and hostile people are viewed as individuals who are easily angered, often irritated, inclined to argument, and cynical and mistrusting in their attitude toward others. Both prospective and cross-sectional studies have provided evidence that there is an association between hostility and CHD. Despite several studies with negative findings (Hearn, Murray, \& Luepker, 1989; Helmer, Ragland, \& Syme, 1991; Leon, Finn, Murray, \& Bailey, 1988; McCranie, Watkins, Brandsma, \& Sisson, 1986), the bulk of the evidence suggests that hostility contributes to an increased risk of CHD (Barefoot, Dahlstrom, \& Williams, 1983; Barefoot, Dodge, Peterson, Dahlstrom, \& Williams, 1989; Dembroski, MacDougall, Costa, \& Grandits, 1989; Dembroski, MacDougall, Williams, Haney, \& Blumenthal, 1985; Haynes, Feinleib, \& Kannel, 1980; Hecker, Chesney, Black, \& Frautschi, 1988; MacDougall, Dembroski, Dimsdale, \& Hackett, 1985; Matthews, Glass, Rosenman, \& Bortner, 1977; Meesters, Muris, \& Backus, 1995; Meesters \& Smulders, 1994; Shekelle, Gale, Ostfeld, \& Paul, 1983; Siegman, Dembroski, \& Ringel, 1987; Williams et al., 1980), especially in younger people (e.g. Kannel, Neaton, \& Wentworth, 1986; Siegman et al., 1987; Williams et al., 1988).

In many studies, feelings of excess fatigue and lack of energy have been found to be among the most frequent precursors of myocardial infarction (MI) and sudden death. Estimates of the percentage of people reporting feelings of fatigue or general malaise before MI or sudden death range between $13 \%$ and $70 \%$ (Alonzo, Simon, \& Feinleib, 1975; Fraser, 1978; Kinlen, 1973; Kuller, 1978; Rissanen, Romo, \& Siltanen, 1978; Wolf, 1969). These feelings reflect a state of both mental and physical exhaustion. Exhausted subjects most commonly complain about feelings of undue tiredness, increased irritability, listlessness, and feelings of demoralization. This state has been labeled as "vital exhaustion" (VE), and is defined as a state characterized by: (1) unusual fatigue and lack of energy; (2) increased irritability; and (3) feelings of demoralization. Three prospective studies (Appels \& Mulder, 1988; Appels \& Otten, 1992; Kop, Appels, Mendes de Leon, de Swart, \& Bär, 1994) and four casecontrol studies (Appels, Falger, \& Schouten, 1993; Falger \& Schouten, 1992; Nelissen-de Vos, 1994; Ranchor, 1994) provided evidence that VE increases the risk of MI in apparently healthy men and women and the risk for a new coronary event after angioplasty. Besides, the associations that were observed proved to be independent of the known risk factors for CHD. Note that, in contrast to hostility, which is defined as a set of stable attitudes and behaviors, vital exhaustion is conceptualized as an endpoint of a process of stress reflecting a breakdown in adaptation to stress.

To date, few non-US studies have addressed the question which elements of hostility increase the risk for MI, and research on the association between hostility and VE is lacking. Therefore, it is unknown whether these two factors are independent or interrelated risk factors. A possible interrelation could take the form of an interaction (indicating for example that only those hostile subjects who become exhausted are at 
increased risk of myocardial infarction) or of a sequential progression of hostility and exhaustion over time (exhaustion leading to a cynical attitude towards other people or vice versa). The existence of a sequential progression over time has been suggested in burnout research. The classic definition of burnout distinguishes three independent components: emotional exhaustion (which shows considerable overlap with VE), depersonalization (which has some overlap with hostility), and reduced personal accomplishment (Maslach \& Jackson, 1986; Pines \& Aronson, 1988). The empirical work of Golombiewski, Munzenrider, and Stevenson (1986) suggests that depersonalization is the first phase of burnout, followed by reduced personal accomplishment, and finally by emotional exhaustion. In contrast, the model developed by Leiter (1993) postulates that emotional exhaustion occurs first, and leads to the development of depersonalization, which, in turn, results in reduced personal accomplishment.

Evidence from various studies on hostility shows that highly hostile persons report low levels of social support, high levels of interpersonal conflict botl at home and at work, and more adverse life events (Barefoot et al., 1983; Hardy \& Smith, 1988; Houston \& Kelly, 1989; Scherwitz, Perkins, Chesney, \& Hughes, 1991; Smith \& Frohm, 1985; Smith, Pope, Sanders, Allred, \& O'Keeffe, 1988). These unfavorable circumstances result in a psychosocial profile that might increase the risk of exhaustion, and, consequently, of disease.

The present case-control study was designed to investigate which elements of hostility increase the risk for MI and to examine the association between hostiiity and VE. A positive relationship between VE and hostility was expected. Furthermore, it was hypothesized that there is a sequential progression over time, that is, that hostility precedes VE. Therefore, it is predicted that, if hostility and VE are entered simultaneously in a multivariate analysis, the discriminating power of hostility will no longer be significant.

\subsection{METHOD}

\section{Subjects and procedure}

A total of 249 males between 35 and 65 years of age from South Limburg, the southernmost region of The Netherlands, took part in the study. The cases comprised a consecutive series of male patients $(N=81$; mean age $55.6 \pm 6.8$ years) with a first clinically documented $\mathrm{MI}$ who were hospitalized in the cardiology units of two regional hospitals between January 1990 and December 1990. The final diagnosis of first MI was based on the clinical history taken by a cardiologist, standard ECG readings, and plasma enzyme assessments. Patients with other major diseases such as cancer were excluded.

Both hospitals were visited once a week, and all new patients with a first MI who were to be discharged from the coronary care unit, were asked to participate by the first author. Each patient was informed of the aim and the procedures of the study. 
Once the patient had agreed to participate, he was requested to sign a written informed consent form. Subsequently, the patient was given a set of questionnaires and was asked to complete the questionnaires as soon as possible and to return them within two weeks after discharge from hospital. Finally, a provisional appointment was made for an interview at home, which was scheduled within 4-6 weeks after discharge from hospital. Altogether, 94 MI cases were contacted, 13 of whom refused. The final response rate was $86 \%$.

In order to obtain a control group, which would be a fair representation of the source population (Miettinen, 1985), a neighborhood control (NC) group was selected consisting of 168 age-matched males (mean age $54.9 \pm 6.9$ years) living on the same or on an adjacent street as the MI patients at the time of the latter's myocardial infarction. The criteria for inclusion that were used for cases were also applied to controls; subjects with previous MI or those having a major disease (e.g. cancer) at the time were excluded. NCs were randomly selected via the telephone directory in January 1990 and May 1991. Once a control subject had agreed to participate, a set of questionnaires was mailed and an appointment for an interview at home was made. A total of 204 eligible NCs were contacted, 168 of whom ultimately participated in the present study.

\section{Instruments}

Hostility was assessed by means of the Dutch versions of the Cook-Medley (Ho) Scale (Cook \& Medley, 1954) and the Buss-Durkee Hostility Inventory (BDHI) (Buss \& Durkee, 1957). The Cook-Medley (Ho) Scale is a 50-item, true-false measure of hostility derived from the MMPI. Ho scores range from 0 to 50 . Generally, high scores on the scale are indicative of a cynical, mistrustful attitude towards other people (Smith \& Frohm, 1985; Smith \& Pope, 1990). Internal consistency of the Ho scale in the present sample was 0.85 (Cronbach's $\alpha$ ). In a follow-up of the present study sample by Neissen (1993), two-year test-retest reliability was found to be $r=0.79$ for the total sample, $r=0.73$ for cases, and $r=0.82$ for controls, which corresponds well with other studies (Barefoot et al., 1983; Shekelle et al., 1983; Smith \& Frohm, 1985).

The Buss-Durkee Hostility Inventory (BDHI) is a self-report questionnaire consisting of 66 true-false items. The BDHI can be divided into seven subscalles measuring several attitudinal styles and reactions (Assault, Indirect Hostility, Irritability, Negativism, Resentment, Suspicion, and Verbal Hostility). Several factor analytic studies have provided evidence of the presence of two distinct factors representing two different types of hostility, wiz. an expressive kind of hostility (primarily defined by the Assault, Indirect Hostility, Verbal Hostility, and Irritability subscales) and an experiential kind of hostility (primarily indexed by the Resentment and Suspicion subscales) (Buss \& Durkee, 1957; Bushman, Cooper, \& Lemke, 1991; Musante, MacDougall, Dembroski, \& Costa, 1989). The present investigation used a true-?-false format. Cronbach's $\alpha$ 's for BDHI Total, the expressive factor and the experiential 
factor were $0.88,0.76$ and 0.85 , respectively, indicating good internal consistency. The study by Neissen (1993) yielded two-year test-retest reliability coefficients of $0.78,0.82$, and 0.76 for the total sample, cases, and controls, respectively.

Vital exhaustion was measured by means of the Maastricht Interview for Vital Exhaustion (MIVE), a 23-item structured interview. In previous studies of vital exhaustion, this state was measured by means of the Maastricht Questionnaire (MQ). Although the MQ has good psychometric properties, van Diest and Appels (1994) showed that its value as a screening instrument for the selection of subjects for experimental research is limited because of a significant number of false positives. In order to exclude these false positives the MIVE has been developed. The interview can be employed to obtain either a dimensional or a categorical classification. The dimensional classification considers exhaustion as a continuum, ranging between a minimum score of 0 and a maximum score of 23 . This dimensional classification can be converted into a categorical classification by using a cut-off score. In addition, in order to be classified "exhausted", subjects should not have had complaints for longer than 18 months (Meesters \& Appels, 1995a, 1995b). This criterion was applied because VE is a state and not a chronic condition. MI patients were asked to indicate how they had felt in the last few months before admission to hospital, while NCs were asked to describe how they had felt during the last month prior to the interview. All interviews were taperecorded and scored independently by the two authors. In the present study, the interview was found to have good reliability, Cronbach's $\alpha$ being 0.90 for cases and 0.86 for controls. The kappa coefficient was 0.95 in the case group and 0.91 in the control group, indicating a high level of interrater agreement (Meesters \& Appels, 1995a, 1995b).

Data on demographic characteristics and cardiovasular risk factors were collected by means of a questionnaire and included age, angina pectoris, hypertension, diabetes mellitus, smoking, and family history of CHD.

Angina pectoris within the last six months before MI or before the interview was assessed by means of the London School of Hygiene Cardiovascular Questionnaire (Rose, Blackburn, Gillum \& Prineas, 1982).

Diabetes mellitus and hypertension were defined as having been treated for these cardiovascular risk factors in the six months prior to MI or the interview.

Smoking was defined as the number of cigarettes smoked per day during the last six months before hospitalization or the interview. Subjects were classified as 1) nonsmokers; 2) moderate smokers (1-19 cigarettes daily); and 3 ) heavy smokers (20 cigarettes or more daily).

Family history of CHD was considered positive if a subject's parent or sibling had ever suffered a fatal or non-fatal MI.

\section{Data Analysis}

Univariate differences in hostility and vital exhaustion were tested using $t$-tests comparing MI cases and neighborhood controls. Separate multiple logistic regressions 
were computed for each of the hostility measures and vital exhaustion in order to test for differences between cases and controls, adjusting for several control variables, employing the SPSS/PC 4.0 (Norusis, 1990). Group status (MI or NC) was used as the dependent variable, while the control group served as the reference group. Hostility was included in the model as a continuous variable, while vital exhaustion was employed as a categorical variable. Persons with an interview score equal to or greater than 8 were classified as "exhausted" if the complaints had not been present for longer than 18 months. Subjects whose complaints had lasted for more than 18 months were labeled "non-exhausted".

Control variables in the multivariate analyses were age, smoking, angina pectoris, hypertension, diabetes mellitus, and family history of CHD. Age was included as a continuous variable; smoking (non-smoking, moderate smoking, and heavy smoking) angina pectoris (present/absent), hypertension (present/absent), diabetes mellitus (present/absent), and family history of CHD (present/absent) were included as categorical variables. Besides, interactions between the various hostility measures and age as well as between VE and age were examined.

The relationship between dimensions of hostility and vital exhaustion was examined using product-moment correlations.

The final logistic models, in which hostility and vital exhaustion are included simultaneously, contained the same control variables as the initial logistic regression analyses. At the same time, the interaction between hostility and VE was explored.

Because of missing data there may be small differences in the numbers of subjects in different tables.

\subsection{RESULTS}

\section{Sample characteristics}

The main demographic and health characteristics of the present sample are shown in table 8.1. There were no significant differences between the case group and the control group with respect to age and self-reported high blood pressure. The mean age of both cases and controls was about 55 years. Current smoking, diabetes mellitus, family history of CHD, and angina pectoris were significantly more prevalent among cases than among controls. 
Table 8.1. Demographic and health characteristics of the sample

\begin{tabular}{|c|c|c|c|}
\hline $\mathbf{N}$ & $\begin{array}{r}\text { MI } \\
\text { (81) }\end{array}$ & $\begin{array}{l}\mathrm{NC} \\
(168)\end{array}$ & \\
\hline Age & $\begin{array}{r}55.6 \\
(\mathrm{sd}=6.8)\end{array}$ & $\begin{array}{r}54.9 \\
(s d=6.9)\end{array}$ & $\begin{array}{l}t=0.71 \\
p=0.48\end{array}$ \\
\hline $\begin{array}{l}\text { Current smoking: } \\
\text { non-smokers } \\
\text { moderate smokers } \\
\text { heavy smokers }\end{array}$ & $\begin{array}{l}23 \\
25 \\
33\end{array}$ & $\begin{array}{r}100 \\
41 \\
27\end{array}$ & $\begin{array}{l}x^{2}=25.4 \\
p=0.000\end{array}$ \\
\hline $\begin{array}{l}\text { Hypertension: } \\
\text { present } \\
\text { absent }\end{array}$ & $\begin{array}{l}17 \\
64\end{array}$ & $\begin{array}{r}32 \\
136\end{array}$ & $\begin{array}{l}\chi^{2}=0.13 \\
p=0.72\end{array}$ \\
\hline $\begin{array}{l}\text { Diabetes mellitus: } \\
\text { present } \\
\text { absent }\end{array}$ & $\begin{array}{r}6 \\
75\end{array}$ & $\begin{array}{r}3 \\
165\end{array}$ & $\begin{array}{l}x^{2}=4.96 \\
p=0.03\end{array}$ \\
\hline $\begin{array}{l}\text { Family history of CHD } \\
\text { present } \\
\text { absent }\end{array}$ & $\begin{array}{l}47 \\
34\end{array}$ & $\begin{array}{l}69 \\
99\end{array}$ & $\begin{array}{l}x^{2}=6.31 \\
p=0.01\end{array}$ \\
\hline $\begin{array}{l}\text { Angina pectoris: } \\
\text { present } \\
\text { absent }\end{array}$ & $\begin{array}{l}18 \\
62\end{array}$ & $\begin{array}{r}11 \\
157\end{array}$ & $\begin{array}{l}x^{2}=8.90 \\
p=0.003\end{array}$ \\
\hline
\end{tabular}

\section{Association of hostility and MI}

Means and standard deviations on the Ho scale and the BDHI are presented in table 8.2. As can be seen, there were no significant differences in mean Ho scores and BDHI Total scores between cases and controls. As was already mentioned in the introductory section, several studies have found hostility to be more predictive of CHD in younger subjects (Siegman et al., 1987; Williams et al., 1988; Williams, Barefoot, \& Shekelle, 1985). To investigate a possible age-dependent association between hostility and MI we carried out multiple logistic regression analyses (on the Ho scale and each of the BDHI scales separately), including an interaction between hostility and age. The results suggested an interaction between Ho scores and age $(\beta=-0.005$, s.e. $=0.003 ; p=0.06)$, indicating that in this study, hostility, as measured by the Ho scale, is associated with MI only in interaction with age. With respect to the $\mathrm{BDHI}$ regressions, none of the interaction terms was found to be statistically significant ( $\mathrm{p}$-values ranging from 0.14 to 0.90 ). 
Table 8.2. Means (and standard dleviations) on each of the hostility measures and the Maastricht Interview for Vital Exhaustion (MIVE) comparing cases $(n=81)$ and controls $(n=168)$ by means of thtest.

\begin{tabular}{lcccccc}
\hline & \multicolumn{7}{c}{ MI } & \multicolumn{2}{c}{ NC } & & \\
Scale & Mean & sd & Mean & sd & t & p(two-tailed $)$ \\
\hline Ho & 22.1 & 8.0 & 20.7 & 7.5 & 1.34 & 0.18 \\
BDHI Total & 50.9 & 18.4 & 48.5 & 17.1 & 0.99 & 0.34 \\
& & & & & & \\
Assault & 7.1 & 3.8 & 6.3 & 3.9 & 1.59 & 0.12 \\
Indirect Hostility & 6.7 & 3.7 & 6.7 & 3.7 & -0.02 & 0.98 \\
Irritability & 8.8 & 4.9 & 8.8 & 4.3 & 0.04 & 0.98 \\
Negativism & 4.9 & 2.4 & 4.4 & 2.3 & 1.42 & 0.16 \\
Resentment & 3.7 & 2.9 & 2.9 & 2.8 & 1.98 & 0.04 \\
Suspicion & 6.5 & 3.7 & 5.4 & 3.8 & 2.13 & 0.04 \\
Verbal Hostility & 13.2 & 5.1 & 13.8 & 5.1 & -0.90 & 0.38 \\
& & & & & & \\
Expressive factor & 35.8 & 14.0 & 35.5 & 12.7 & 0.14 & 0.89 \\
Experiential factor & 10.3 & 5.8 & 8.4 & 5.9 & 2.34 & 0.02 \\
Mrve & 7.8 & 6.0 & 3.6 & 4.0 & 5.65 & 0.00 \\
\hline
\end{tabular}

Note: $H_{0}=$ Cook-Medley Hostillity Scale; $\mathrm{BDHI}=$ Buss-Durkee Hostility Inventory.

In order to gain more insight into the relationship between Ho scores and age, we divided the sample into three different age strata [35 to $49(n=61), 50$ to 59 $(\mathrm{n}=112)$, and 60 to $65(\mathrm{n}=75)]$. This showed that cases only had significantly higher Ho scores in the youngest age group $(24.5 ;$ sd 8.7 vs. 20.1 ; sd $7.6 ; p<0.05)$. Over 50 years of age mean Ho scores did not differ between the case group and the control group.

As was said before, cases and controls did not differ significantly with respect to level of general hostility. Inspection of the separate hostility components, as assessed by the BDHI, shows that MI cases had higher Resentment $(3.7$; sd 2.9 vs. 2.9; sd $2.8 ; \mathrm{p}<0.05)$. Suspicion (6.5; sd 3.7 vs. 5.4 ; sd $3.8 ; \mathrm{p}<0.05)$, and BDHI experiential factor $(10.3$; sd 5.8 vs. 8.4 ; sd $5.9 ; p<0.03$ ) scores than controls (see table 8.2 ). All other BDHI dimensions did not discriminate between cases and controls.

The outcomes of the multivariate analyses are displayed in table 8.3. The series of multiple logistic regression models, adjusted for control variables, yielded results which are fairly similar to the univariate findings. Only Suspicion $(p=0.05)$ and the experiential factor of the BDHI $(\mathrm{p}=0.03)$ proved to be significantly associated with MI, while Resentment lost its discriminating power $(p=0.14)$. None of the other associations was statistically significant. 
Table 8.3. Logistic regression models for each of the hostility measures and vital exhaustion separately, adjusted for age, smoking, angina pectoris, hypertension, diabetes mellitus, and familly history of CHD

\begin{tabular}{|c|c|c|c|c|}
\hline Scale & $\beta$ & se & OR & $p$ (two-twiled $)$ \\
\hline Ho * Age & -0.005 & 0.003 & & 0.06 \\
\hline BDHI Total & 0.003 & 0.009 & 1.00 & 0.73 \\
\hline Assault & 0.030 & 0.040 & 1.03 & 0.45 \\
\hline Indirect Hostility & -0.007 & 0.043 & 0.99 & 0.88 \\
\hline Irritability & -0.020 & 0.035 & 0.98 & 0.59 \\
\hline Negativism & 0.040 & 0.064 & 1.04 & 0.57 \\
\hline Resentment & 0.083 & 0.056 & 1.09 & 0.14 \\
\hline Suspicion & 0.077 & 0.040 & 1.08 & 0.05 \\
\hline Verbal Hostility & -0.044 & 0.031 & 0.96 & 0.16 \\
\hline Expressive factor & -0.005 & 0.011 & 1.00 & 0.68 \\
\hline Experiential factor & 0.042 & 0.020 & 1.04 & 0.03 \\
\hline Vital Exhaustion & 2.05 & 0.433 & 7.80 & 0.00 \\
\hline
\end{tabular}

\section{Association of vital exhaustion and MI}

The results of the univariate analysis indicate that MI patients had significantly higher mean scores on the Maastricht Interview for Vital Exhaustion (MIVE) than the control group (7.8; sd 6.0 vs. 3.6 ; sd $4.0 ; p<0.001$, see table 8.2). Subsequent multiple logistic regression analysis corroborates the univariate findings. Table 8.3 shows that vital exhaustion, as measured by the MIVE, is significantly associated with MI, while adjusting for age, smoking, angina pectoris, hypertension, diabetes mellitus, and family history of $\mathrm{CHD}(\mathrm{OR}=7.80 ; \mathrm{p}<0.001)$.

\section{Relationship between hostility and vital exhaustion}

Vital exhaustion generally seems to be related to hostility, which becomes clear from the product-moment correlations between the interview and the Ho scale and the different BDHI subscales presented in table 8.4. The strongest positive relationships were those between vital exhaustion and BDHI Total, Irritability, and Resentment, both in the MI case group and in the control group. In addition, vital exhaustion was found to be significantly related to both expressive and experiential hostility in cases and to experiential hostility in controls. Negativism and verbal hostility seem to be unrelated to vital exhaustion. On the whole, associations between interview scores and various dimensions of hostility appear to be somewhat more robust in the MI case group. 
Table 8.4. Pearson product-moment correlations between the Maastricht Interview for Vital Exhaustion (MIVE) and each of the hostility variables

\begin{tabular}{lll}
\hline & $\begin{array}{l}\text { Cases } \\
(\mathbf{n}=80)\end{array}$ & $\begin{array}{l}\text { Controls } \\
(\mathbf{n}=166)\end{array}$ \\
\hline Ho & $0.24^{*}$ & $0.33^{* * *}$ \\
BDHI Total & $0.42^{* * *}$ & $0.25^{* * *}$ \\
Assault & $0.44^{* * *}$ & $0.18^{*}$ \\
Indirect Hostility & $0.37^{* * *}$ & 0.13 \\
Irritability & $0.41^{* * *}$ & $0.30^{* * *}$ \\
Negativism & 0.12 & 0.06 \\
Resentment & $0.37^{* * *}$ & $0.39^{* * *}$ \\
Suspicion & 0.08 & $0.17^{*}$ \\
Verbal Hostility & 0.17 & -0.01 \\
Expressive factor & $0.41^{* * *}$ & $0.19^{*}$ \\
Experiential factor & 0.20 & $0.32^{* * *}$ \\
\hline
\end{tabular}

$* \mathrm{p}<0.05 ; * * \mathrm{p}<0.01 ; * * * \mathrm{p}<0.001$

\section{Association of hostility and vital exhaustion and MI}

To test the association between hostility and vital exhaustion we performed additional multiple logistic regression analyses including exhaustion and hostility simultaneously in the same logistic model. First of all, a possible interaction between hostility and VE was explored. No significant interaction between any of the hostility measures and VE emerged. Therefore, the interaction term was left out from all subsequent regression analyses. The results are displayed in table 8.5.

Table 8.5. Results of three separate multiple logistic regression analyses including vital exhaustion and hostility measures in the same model, adjusting for age, smoking, angina pectoris, hypertension, diabetes mellitus, and fannily history of CHD

\begin{tabular}{llllc}
\hline Measure & $\beta$ & se & OR & P(two-tailed) \\
\hline Vital Exhaustion & 2.01 & 0.434 & 7.48 & 0.00 \\
Suspicion & 0.069 & 0.042 & 1.07 & 0.10 \\
Vital Exhaustion & 1.99 & 0.435 & 7.33 & 0.00 \\
Experiential factor & 0.036 & 0.021 & 1.04 & 0.08 \\
\hline
\end{tabular}

Only the findings concerning those hostility measures which were found to be significantly associated with MI in the previous analyses are presented. In all analyses, VE discriminated strongly between cases and controls, while none of the hostility mea- 
sures did. To be more specific, both suspicion and experiential hostility, which discriminated significantly between cases and controls at both univariate and multivariate level, lost their discriminating power.

\subsection{DISCUSSION}

The main aim of the present study was to explore which elements of hostility increase the risk for MI and to test the hypothesis that elements of hostility increase the risk for $\mathrm{MI}$ because they increase the risk of becoming exhausted.

The results showed that hostility, defined as the total scores on the Ho scale and the $\mathrm{BDHI}$, is not significantly related to $\mathrm{MI}$. However, in the present study the presence of an interaction between the Ho scale and age was suggested, indicating that hostility, as measured by the Ho scale, is only associated with an elevated risk of MI in younger subjects ( $<50$ years). In contrast, no interaction was observed between any of the BDHI measures and age. This discrepancy may be attributed to the fact that the Ho scale and the BDHI are only moderately related and probably measure different kinds of hostility (Meesters, Muris, \& Backus, 1995). Moreover, several studies have pointed out that hostility is a multidimensional construct (Bendig, 1962; Buss \& Durkee, 1957; Bushman et al., 1991; Musante et al., 1989; Sarason, 1961) and that only some aspects of hostility may confer risk for the development of CHD (Siegman, Anderson, Herbst, Boyle, \& Wilkinson, 1992; Siegman et al., 1987). Both univariate and multivariate results of the separate BDHI measures provide support for these findings. MI patients scored significantly higher on Resertment, Suspicion, and experiential hostility. All other scales revealed no significant differences in mean scores between cases and controls. The multivariate results are generally in line with the univariate findings. In conclusion, especially experiential hostility was found to discriminate significantly between cases and controls, while adjusting for age, smoking, angina pectoris, hypertension, diabetes mellitus, and family history of CHD.

Vital exhaustion, as measured by means of the MIVE, proved to be strongly associated with MI. Cases scored twice as high on the interwiew as control subjects. Multivariate analysis indicated that this strong association could not be attributed to angina pectoris or to the relationship between exhaustion and some of the classic risk factors of CHD.

The above findings confirm our hypothesis that hostility and vital exhaustion are two interrelated psychosocial factors. This association is not synergistic, however, because no significant interaction between VE and any of the hostility measures emerged. Additional analyses revealed that, when VE and hostility were included simultaneously in the same model, hostility lost its discriminating power, while the association between VE and MI remained significant, thus providing evidence of our hypothesis of a sequential relationship between hostility and VE. 
Due to the retrospective design of the present study the observed associations between hostility, vital exhaustion and $\mathrm{MI}$ should be treated with caution. The differences in levels of hostility and vital exhaustion may be attributable to recall bias. This "search for meaning", which happens when people try to find an explanation for their disease, may influence memories of the past and thus lead to an overestimation of the risk associated with hostility and vital exhaustion. However, it is unclear why experiential aspects of hostility are probably more sensitive to a search for meaning than expressive hostility, which did not discriminate between cases and controls. As to the mental precursors of MI (e.g. feelings of tiredness and exhaustion), experimental investigations have shown that this search for explanations is not focused on premonitory symptoms but rather on risk behaviors considered to be causal (Croyle \& Sande, 1988). This means that people may not be looking for the fatigue prior to MI, but rather for the event(s) that caused the tiredness.

Selection bias may have affected the results of the present study. There were no differences between participating and non-participating cases, however, with respect to age, smoking, high blood pressure, and angina pectoris. It was observed several times that control subjects who were not working at the time were somewhat more willing to participate. Therefore, the presence of some degree of selection bias with regard to the control group cannot be ruled out completely.

The accuracy of the estimated associations may also have been affected by the imperfect assessment of control variables. Hypertension was measured by means of self-reports, which may have been subject to a higher detection rate among subjects who saw their physician because of fatigue complaints, resulting in an underestimation of the risk.

In the present study, no information about cholesterol levels was available. Although it is common in health and behavioral medicine research to control for baseline plasma cholesterol levels, statistical adjustment of cholesterol in multivariate analyses of the association between hostility and health (e.g. MI) may lead to an underestimation of the associated risk, because of the reported relationship between hostility and lipids (Smith, 1992). As it has been demonstrated that vital exhaustion is not associated with cholesterol in males (Appels \& Mulder, 1988), it is unlikely that the inclusion of cholesterol in the logistic models would have produced noticeably different outcomes.

The observed odds ratio of vital exhaustion associated with MI may also be attributed to subclinical heart disease. The assessment of angina pectoris was based on self-reports and no data were available on the left ventricular ejection fraction. Nevertheless, we do not believe that exhaustion is associated with $\mathrm{MI}$ because it is a reflection of subclinical heart disease, as Kop has shown that VE is not related to left ventricular ejection fraction and only marginally to the extent of coronary atherosclerosis (Kop, 1994). Besides, the observed odds ratio of 7.80 is similar to the relative risk of $\mathrm{MI}$ in the first year of follow-up (10.01) reported in a previous prospective study (Appels \& Mulder, 1989). 
In sum, the present study has shown that both hostility and wital exhaustion are associated with increased risk of MI. Furthermore, it was demonstrated that hostility and vital exhaustion are interrelated. This finding gives rise to several questions about the nature of this association. Firstly, it is possible that exhausted people become more hostile. In this sense, hostility might increase the risk for MI because it is part of the exhaustion syndrome. However, vital exhaustion is defined as a state, whereas hostility is considered to be a trait characteristic. Secondly, subjects exhibiting high levels of hostility my, under certain conditions, be more at risk of becoming exhausted. Finally, guided by Redford B. Williams' model of hostility (1994), the following dynamic model is proposed: If a hostile person is no longer able to cope efficiently with a problem which chronically provokes hostile responses, such a person will become exhausted. In turn, exhaustion will lead to increased irritability and cynicism. In other words, hostility and exhaustion are believed to operate in a mutually reinforcing way.

Naturally, the present study is unable to give definitive answers to all these questions. It clearly shows, however, that it is very important for future research to address the association between hostility and vital exhaustion, two significant psychosocial risk factors of coronary heart disease. The study of the association between these two factors together and CHD seems promising and fruitful for the further unraveling of the mechanism(s) underlying $\mathrm{CHD}$ and other diseases. 



\section{CHAPTER 9}

\section{GENERAL DISCUSSION}

In the preceding chapters, the empirical results of a case-control study of psychosocial risk factors of cardiovascular disease have been presented. The present study, in particular, investigated whether hostility and vital exhaustion are risk factors of cardiovascular disease. In addition, the childhood origins of hostility were explored. Finally, a possible interrelationship between hostility and vital exhaustion was examined.

The aim of this final chapter is to summarize the main findings of the present study and to interpret them in light of other research. Implications for future directions of research are allso discussed. The first section deals with the association between hostility and myocardial infarction (MI). The following section discusses the role of vital exhaustion in the etiology of MI and the development of a new instrument to measure the mental precursors of MI. Next, the findings regarding the association between hostility and vital exhaustion are evaluated. Finally, implications for future research and suggestions for possible intervention and prevention strategies are discussed.

\subsection{RELATIONSHIP BETWEEN HOSTILITY AND MYOCARDIAL INFARCTION}

Both cross-sectional and prospective evidence of an association between hostility and coronary heart disease (CHD) has primarily been provided by studies conducted in the United States (for an extensive review of studies see Helmers, Posluszny, \& Krantz, 1994). However, outside the US few studies have investigated the hypothesis that chronic hostility may promote the development of CHD. Therefore, the present case-control study was designed to test the association between hostility and CHD in the Netherlands. Chapters 2 and 3 present the results of this study, in which hostility, as assessed by the Cook-Medley Hostility (Ho) Scale and the Buss-Durkee Hostility Inventory (BDHI), is related to first myocardial infarction (MI) in adult men. The Ho scale is the hostility questionnaire used the most frequently in behavioral medicine research by far, whereas the BDHI is one of the most comprehensive measures of hostility, which does not only give an index of general hostility, but also distinguishes several different components of hostility. This is relevant since the findings of several studies have indicated that hostility is a multidimensional construct (Bendig, 1962; Bushman, Cooper, \& Lemke, 1991; Buss \& Durkee, 1957; Edmunds \& Kendrick, 
1980; Musante, MacDougal1, Dembroski, \& Costa, 1989; Sarason, 1961; Siegman, Dembroski, \& Ringel, 1987) and that it is likely that not all dimensions contribute to elevated risk of future CHD (Siegman et al., 1987). In the present study, it was found that general hostility, as defined by the total scores on the Ho scale and the BDHI, is not significantly related to MI. However, subsequent multivariate analysis suggested an interaction between Ho scores and age, that is, in the current sample, hostility (as measured by the Ho scale) only seems to be associated with increased risk of MI in people under 50 . This finding is in agreement with previous studies, which also found the association between hostility and CHD to be age-dependent (Dembroski, MacDougall, Costa, \& Grandits, 1989; Siegman et al., 1987). In contrast, no interaction was observed between BDHI Total score or any of the BDHI subscales and age. A possible explanation for these conflicting findings is that the $\mathrm{Ho}$ scale and the BDHI tap different aspects of hostility. Our observation in the present study that the Ho scale and the BDHI correlate only moderately supports this assumption.

As was indicated before, the present study was also designed to investigate which dimensions of hostillity increase the risk of MI. The overall finding was that the experiential aspects of hostility, represented by the BDHII Resentment and Suspicion subscales, appeared to be significantly associated with first MI in adult Dutch males. No relationship was found between expressive hostility, which is mainly reflected by items from the BDHI Assault, Indirect Hostility, Irritability, and Verbal Hostility subscales, and MI. Interestingly, it was shown that the relationship between Ho scores and the BDHI Experiential Hostility factor was significantly stronger than the correlation with the BDHI Expressive Hostility factor (see Chapter 3). This implies that, in the current study, people exhibiting high levels of resentment, suspicion, and distrust and who view others cynically as mean, nasty, selfish and dishonest are at elevated risk of MI. It is noteworthy that these results disagree with the findings of others, in particular Siegman and his colleagues (for a review of studies see Siegman, 1994b). On the basis of the outcome of a study of angiography patients they reported that expressive hostility confers risk of coronary artery disease (CAD), but that experiential hostility is associated negatively with severity of CAD (Siegman et al., 1987). Several possible explanations of the discrepancies between the findings of different studies will be briefly summarized here. First, mast of the BDHI studies related dimensions of hostility to cardiovascular reactivity in healthy and relatively young subjects free of C.AD (Siegman, Anderson \& Berger, 1990; Siegman, Anderson, Herbst, Boyle, \& Wilkinson, 1992; Suarez \& Williams, 1990). So far, the study by Siegman et al. (1987) is the only one in which the relationship between different dimensions of hostility (as measured by the BDHI) and coronary disease endpoints (i.e. the extent of occlusion in coronary arteries) was examined. This study also observed a significant positive correlation between anxiety (or neuroticism) and experiential hostility, whereas expressive hostility proved to be unrelated to indices of neuroticism. Costa, Zonderman, McCrae, and Williams (1986) showed that neurotic individuals are more likely to report hypochondriacal cardiovascular-like pains with- 
out any evidence of CHD. Consequently, these persons are more likely to be referred for coronary angiography. This means that the observed inverse association between experiential hostility and CAD severity may be artifactual due to a substantial number of neurotic, but healthy, subjects in the angiography sample of the study by Siegman et all. (1987).

Second, unlike Siegman and his co-workers (1987), who chose the extent of atherosclerosis as the clinical endpoint in their study, the present investigation employed the hard clinical endpoint of MI. Therefore, it is questionable whether the findings of the two studies are comparable.

Third, the difference in findings may be a reflection of possible cross-cultural differences. For example, although the Type A behavior pattern has been shown to have cross-cultural validity (Appels, Jenkins, \& Rosenman, 1982; Hayanao et al., 1989; Sykes, Haertel, Gostautas, \& Evans, 1992; Wrzesniewski, Forgays, \& Bonaiu10, 1990), the study by Sykes and his co-workers (1992) also revealed substantial variations between populations in levels of risk associated with the different Type $A$ components. In addition, although Appels and his colleagues (1982) found some evidence of cross-cultural validity of the Jenkins Activity Survey (JAS), they also observed that the Type A behavior pattern is less pronounced and less well-defined in The Netherlands than in the United States. The picture that emerged during the Structured Interviews (SIs) held in the present study, are in line with this observation. Therefore, cross-cultural variations may also account for the inconsistencies between the findings of the present study and those of other studies of hostility.

Fourth, most of the studies of the possible role of psychosocial factors in the etiology of CHD employed subjects who are in the middle of their professional career. In the present study, however, nearly half of the subjects were retired or did not work anymore because of incapacity for work, which may, to some unknown degree, have led to different perceptions of their own behavior.

\subsection{DEVELOPMENTAL ASPECTS OF HOSTILITY}

So far, the developmental antecedents of hostility have not been studied extensively Studies of the childhood determinants of hostility can be divided into heritability studies, focusing on genetic factors, and investigations placing emphasis on the influence of early environmental factors such as parental rearing behavior. As part of this thesis, two separate studies of the relationship between perceived parental rearing behavior and individual differences in hostility were carried out. The results of these studies were discussed in chapters 4 and 5. On the whole, the findings of the two studies are fairly similar and suggest that family characteristics play a role in the development of hostility. Highly hostile subjects view their parents as more rejective and giving less emotional warmth. Furthermore, perceived parental rejection seems to be the strongest predictor of an individual's level of hostility. Although it is impossible to draw definite conclusions on the basis of the findings of these studies, be- 
cause parental rearing attitudes and behavior were assessed retrospectively, these findings may still be meaningful as they were yielded by studies using quite different samples of subjects: a sample of middle-aged males (mean age about 55 years) mainly with little education on the one hand, and a sample of highly educated young adults (both men and women) with a mean age of 21 years on the other. In conclusion it can be said that, despite the abovementioned methodological limitations, the studies presented in chapters 4 and 5 provide evidence in support of the theory that hostility is rooted in childhood and that parental rearing behavior may influence its development.

\subsection{VITAL EXHAUSTION AND MYOCARDIAL INFARCTION: THE DEVELOPMENT OF AN INTERVIEW}

The relationship between vital exhaustion (VE) and myocardial infarction is the main subject of Chapters 6 and 7. Several studies, both prospective and cross-sectional, have indicated that VE may increase the risk of myocardial infarction. In these studies, the Maastricht Questionnaire (MQ), a self-report inventory, was employed to measure VE. Although the MQ was found to have satisfactory reliability and validity, some important shortcomings also emerged. Especially van Diest and Appels (1994) have shown that its value as a screening instrument for the selection of exhausted and non-exhausted subjects was limited because of a substantial percentage of false positives. Therefore, a structured interview to measure VE was designed. Chapter 6 reports on the development of this interview, which is labeled the Maastricht Interview for Vital Exhaustion (MIVE). The interview and the questionnaire were compared with respect to their validity. Since a gold standard against which the validity of both instruments could be measured is lacking, their capacity of discriminating between cases and controls was taken as the external criterion. The results of the comparison clearly showed that the interview had more discriminating power than the questionnaire and that elevated MQ scores do not necessarily reflect a state of VE. Naturally, this comparison of the interview and the questionnaire should be interpreted with caution. First, due to the design of the study the interviewer was acquainted with the cardiac status of the interviewee, which may have affected the discriminating power of the interview. Second, although the choice of a score between 7 and 8 as the cut-off point as well as the decision to label subjects who reported having experienced feelings of exhaustion for more than 18 months non-exhausted were not arbitrary, they remain open to debate.

The main aim of Chapter 7 is to present data on the psychometric qualities of the MIVE. This interview was found to have good reliability, as is indicated by high Cronbach's $\alpha$ and kappa coefficients. Furthermore, the observation that VE, as measured by the interview, discriminated MI cases significantly from neighborhood. control subjects after adjustment for angina pectoris and the classic risk factors, is 
proof of the validity of the interview, that is, valid assessments of the mental precursors of MI can be made with the MIVE.

However, some reservations should be made regarding the data on the reliability and validity of the interview. As was indicated before, it was impossible to score interviews blind due to the retrospective nature of the study and the set-up of the interview. This may have influenced the observed associations to some extent. However, all questions are phrased in a quite straightforward and unequivocal manner. Therefore, it seems unlikely that the scoring was strongly biased by knowledge of the disease status. Besides, in the case of obscure replies a symptom was strictly scored as negative in order to avoid ambivalent answers being scored as positive in cases and negative in controls.

\subsection{HOSTILITY, VITAL EXHAUSTION AND MYOCARDIAL INFARCTION}

During the past fifteen years, both prospective and cross-sectional studies have found hostility and vital exhaustion to be associated with CHD. In previous chapters it was demonstrated that both vital exhaustion and experiential hostility were associated with elevated risk of first MI, even after adjustment for the classic rist factors of CHD. However, the relationship between these two risk indicators had not been investigated before. Chapter 8 presents data on the association between hostility and vital exhaustion. The results indicate that hostility and vital exhaustion are two interrelated psychosocial factors. This interrelation was not synergistic, as no significant interaction between hostility (as defined by the separate hostility scales) and VE was found. However, when hostility and VE were included simultaneously in multiple logistic regression analyses hostility lost its discriminating power. The association between vital exhaustion and MI, on the other hand, remained highly significant. These findings suggest a sequential relationship between hostility and $\mathrm{VE}$, that is, the risk for cardiovascular disease conferred by hostility might be due to the fact that highly hostile people are at increased risk of becoming exhausted.

\subsection{METHODOLOGICAL LIMITATIONS}

Although potential methodological shortcomings of the present study have already been discussed at length in Chapters 2 through 8 , they are summarized briefly here in order to put the results in perspective. In the present study, a case-control design was employed. Of all research designs within the field of epidemiology, case-control studies have the worst reputation. There is no denying the fact that case-control studies leave more room for bias and incorrect inference than any other type of research. Furthermore, given its retrospective nature, this design does not allow of 
causal inferences. Nevertheless, as the outstanding epidemiologist Rothman (1986) stated in his book Modern epidemiology: "a case-control study can conveniently provide information on a wide range of potentially etiologic exposures that might relate to a specific disease" and "the poor reputation suffered by case-control studies stems more from their inept conduct than from any inherent weakness in the conceptual approach" (p. 68-69).

Let us now turn to some major methodological issues that apply to the present case-control study. First of all, due to retrospective data collection, recall bias, or "search for meaning", may have affected the results. Recall bias may occur when cases are likely to overestimate their own risk factor levels in order to find an explanation for their disease. In the present study, we chose to use a neighborhood control group, which has both advantages and disadvantages (e.g. Kopec \& Esdaile, 1990; Ryu, Thompson, \& Crouse, 1989 ; Wacholder, Silverman, McLaughlin, \& Mandel, 1992). According to Sackett (1979), studies including healthy community controls are more susceptible to recall bias than studies using hospital controls. At the same time, neighborhood controls are more likely to be free of serious disease, which may be related to exposure (i.e. hostility and vital exhaustion). Furthermore, with respect to socioeconomic status, neighborhood controls are probably more similar to cases than hospital control subjects (Vernick, Vernick, \& Kuller, 1984). Finally, in the case group, the space between the MI and assessment was limited to 4-6 weeks in order to reduce the risk of recall bias. Although the possibility of recall bias cannot be ruled out completely, there is no obvious reason to assume that the results of the present study were affected seriously by this process of "search for meaning". First, in the present study, experiential hostility discriminates cases from controls, whereas expressive hostility does not. However, it is not clear why experiential hostillity is more likelly to be influenced by recall bias than expressive hostility. Second, regarding vital exhaustion experimental research has indicated that a search for meaning is not directed at premonitory symptoms but rather at risk behaviors conceived as causal (Croyle \& Sande, 1988). Consequently, it is likely that people are not looking for feelings of fatigue preceding MI, but rather for the event(s) that may have caused these feelings. But most importantly, the estimated relative risk of VE associated with MI is quite similar to the relative risk of $\mathrm{MI}$ in the first year of follow-up reported in the prospective RCSS (Appels \& Mulder, 1988).

Another potential pitfall of case-control designs is the possibility of selection bias, which occurs when cases and controls are differentially selected for participation in a study. This may also apply to the present study. However, no differences emerged between participating and non-participating subjects as to age, current smoking, hypertension, angina pectoris, diabetes mellitus, and family history of CHD. Concerning neighborhood controls, it was occasionally observed that older persons and/or those with jobs tended to be somewhat less willing to participate; this may have influenced the current findings to some unknown degree.

Third, the accuracy of the reported associations throughout the present thesis may also have been affected by the retrospective and imperfect assessment methods of 
(control) variables. For example, both studies dealing with the role of the family environment in the development of hostility (see Chapters 4 and 5) relied on retrospective measurement of parental attitudes and behaviors. Therefore, it could simply be argued that hostile subjects have more negative memories of their parents' behavior due to their negative, resentful view of their environment in general. However, a recent 4-year follow-up study of hostility in children has shown that characteristics of the environment (i.e. level of support and conflict) do indeed promote the development of hostility (Woodall \& Matthews, 1993). In addition, the strength of the association between VE and MI gives rise to some concern. Note that it was impossible to control for subclinical heart disease. Angina pectoris was measured by means of a non-clinical method and data on the left ventricular ejection fraction were not available. However, the study by Kop (1994) indicated that VE is not related to left ventricular ejection fraction and only marginally to the extent of coronary atherosclerosis. Hence, it seems unlikely that the observed odds ratios were seriously influenced by the impossibility of adjustment for subclinical heart disease. Nevertheless, definite conclusions cannot be drawn because knowledge of the exact pathoplnysiological processes underlying the association between feelings of exhaustion and the acute coronary syndromes is lacking. It is still possible that VE does not only reflect a breakdown in adaptation to stress, but is also a pre-clinical marker of coronary artery disease (CAD) itself, which is not expressed by angina pectoris, impaired left ventricular ejection fraction or the extent of atherosclerosis. In this connection, current advances in the understanding of the possible biological mechanisms underlying CAD suggest that psychoneuroimmunological factors may play a role in the development of atherosclerosis.

\subsection{CONCLUSIONS}

Bearing in mind the reservations expressed in the previous section, it still seems worthwile presenting a model in which the main conclusions of the present thesis and the findings of other investigations are integrated.

First, the childhood origins of hostility are represented diagrammatically in Figure 9.1. The results of the present thesis suggest that family environment factors (i.e. parental rejection and lack of parental emotional warmth) contribute to the development of hostility (Chapters 4 and 5). These findings are in line with those of other studies on the potential influence of the early family environment on the development of hostility (e.g. Woodall \& Matthews, 1993). This does by no means imply that other influences, such as genetic factors, are of minor importance in the etiology of psychological characteristics such as hostility (for an extended review see e.g. Plomin \& Bergeman, 1991). For example, a study of twins reared apart by Hur and Bouchard (1995), which was completed only recently, reveals that there is a significant biological basis for individual differences in perceptions of family environments. At present, there is a widespread conviction that genetically based psychological 
characteristics (in this case hostility) and environmental factors are reciprocally related (Scarr \& McCartmey, 1983).

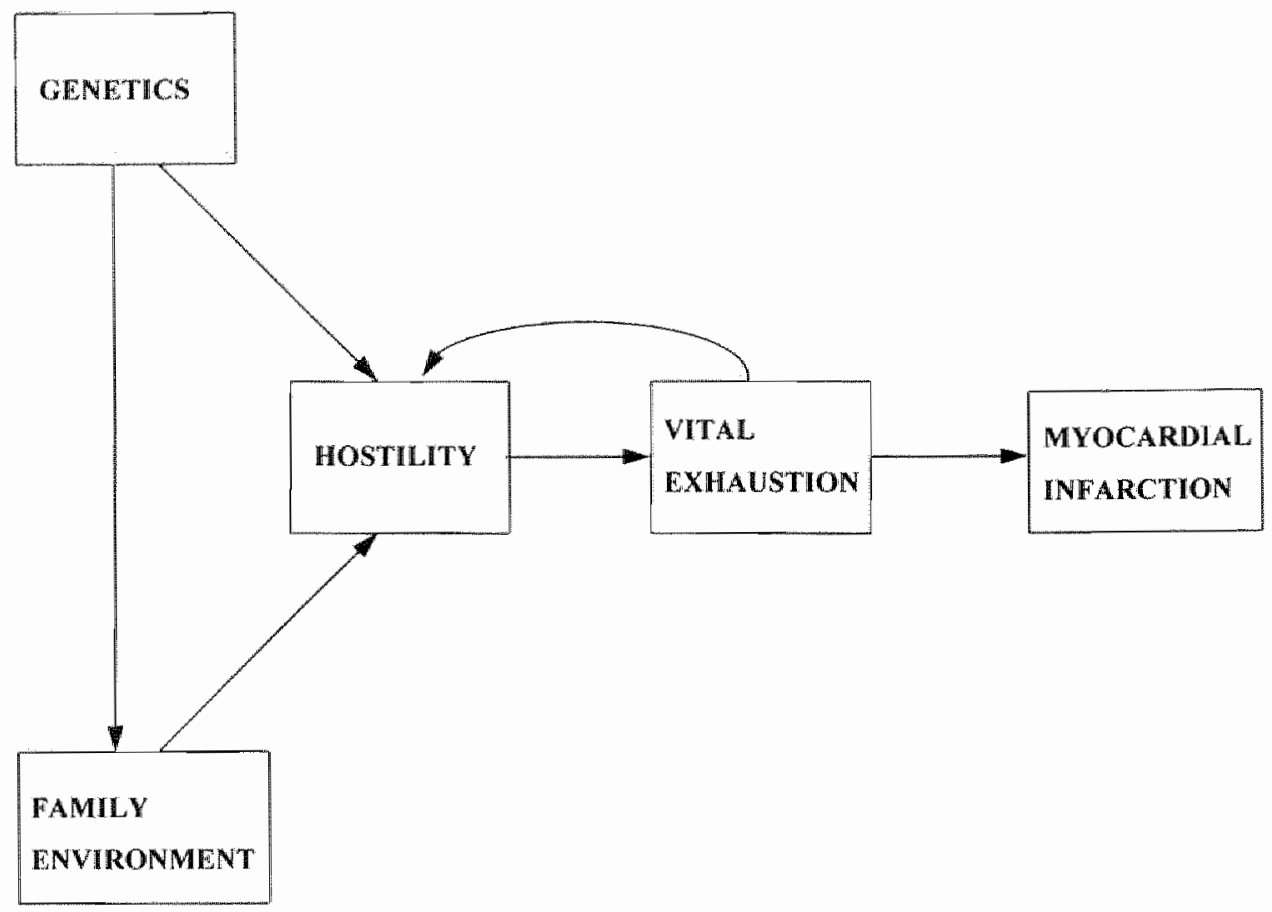

Figure 1. Hostility, vital exhaustion, and myocardial infarction in a life-span perspective

Second, it has been amply reported in the literature that hostility is associated with a greater risk of CHD and other serious diseases. The results of the present study show that the experiential dimension of hostility is a risk indicator of first myocardial infarction in adult males. That is, subjects characterized by high levels of resentment, suspicion, and distrust, who basically view others as selfish, mean and dishonest are at elevated risk for developing CHD, when compared to their non-hostile counterparts (Chapters 2 and 3 ).

Third, given the fact that both (experiential) hostility and vital exhaustion prove to be significantly associated with $\mathrm{MI}$ in the present study, the question arises what the nature of the relationship between the psychological trait of hostility and a state of vital exhaustion is. The present study enabled us to test the association between these psychosocial factors (Chapter 8). The results indicated that hostility lost its discrimi- 
nating power when VE was controlled for in a multivariate model, suggesting a sequential relationship between hostility and VE. It has previously been demonstrated that highly hostile individuals perceive low levels of social support, experience high levels of interpersonal conflict at home and in the working environment, and report more stressful life events (Barefoot, Dahlstrom, \& Williams, 1983; Hardy \& Smith, 1988; Houston \& Kelly, 1989; Scherwitz, Perkins, Chesney, \& Hughes, 1991; Smith \& Frohm, 1985; Smith, Pope, Sanders, Allred, \& O'Keeffe, 1988). Together, these adverse features form a psychosocial profile that may place subjects at increased risk of exhaustion, and as a result, of MI. As Figure 9.1 illustrates, it is possible that a highly hostile person who is no longer able to cope effectively with a problem which chronically elicits hostile responses, will become exhausted in the end. In turn, once exhausted, this person will react with even more irritability, anger, and cynicism. thereby creating a downward spiral.

\subsection{IMPLICATIONS FOR FUTURE RESEARCH AND SUGGESTIONS FOR PREVENTION AND INTERVENTION}

It was already indicated in the introduction (Chapter 1) that studies of the association between hostility and CHD using a behavioral index of hostility (i.e. SI) have produced a more consistent pattern of results than studies relying on self-report paperand-pencil hostility questionnaires. One of the reasons why the relationship between self-reported hostility and CHD is more inconsistent may be the use of different assessment devices across studies. It has repeatedly been suggested that different selfreport hostility measures, such as the Ho Scale and the BDHI, measure different aspects of the multidimensional hostility construct. Furthermore, there is a possibility that not all aspects of hostility are linked to CHD. The divergent findings concerning Cook-Medley and Buss-Durkee hostility presented in this thesis are in line with this explanation (see Chapters 2 and 3 ). In view of the difficulties inherent in self-report measures, the question arises whether it would be wise to use questionnaires in future research. The answer is plainly affirmative. It would be unwise to discard self-report as an instrument for the assessment of hostility, because this method will obviously be needed in future research for the following reasons: it is easy to administer and less time-consuming, and therefore relativelly less expensive, than an interview. It will, therefore, undeniably be necessary for future research to further refine assessment devices, giving special attention to the construct validity of the separate measures.

In the present thesis, a new instrument to assess the mental precursors of MI was introduced. Although the overall findings were promising in the sense that the interview was found to be a reliable instrument and to permit valid assessment of the state of vital exhaustion, future research attempting to accumulate further empirical evidence of the psychometric qualities of this interview would be welcome. 
On the basis of the findings presented in Chapter 8 , a model including hostility, vital exhaustion, and MI was postulated (see Figure 9.1). This model specifically supposes that highly hostile individuals are more at risk for MI due to their increased risk of becoming exhausted. Naturally, a real test of this model should only be performed in future research with an appropriate prospective design.

Finally, the possible implications of the present study for intervention and prevention programs wiil be outlined here. The development of such programs may be based on two distinct viewpoints. First, it would be possible to implement interventions aimed at the modification of hostility in patients who are at high risk of developing CHD. The benefits of such interventions have already been demonstrated by Friedman and his colleagues (1986), who showed that the three-year incidence of recurrent myocardial infarction was significantly lower in patients who had received Type A modification treatment.

The second, and probably the most difficult (but nevertheless very important) challenge for the future would be to aim efforts in the area of prevention at children and adolescents. In keeping with previous studies, the results of the present investigation suggest that factors in the early family environment (i.e. parental rearing behavior) contribute to the development of hostile attitudes and behaviors in children which place them at risk for future CHD. Additional longitudinal research will, of course, be necessary for a clear understanding of the role of parental child-rearing attitudes and behaviors in the development of individual differences in hostility. This understanding could provide the starting-point for future intervention programs aimed at preventing or changing the development of hostile attitudes and behaviors in children, which will, hopefully, bring about a reduction in the risk of developing CHD in later life. 


\section{REFERENCES}

Alexander, F. (1939). Emotional factors in essential hypertension. Psychosomatic Medicine, 1, 175-179.

Allred, K.D., \& Smith, T.W. (1991). Social cognition in cynical hostility. Cognitive Therapy and Research, 15, 399-412.

Almada, S.J., Zonderman, A.B., Shekelle, R.B., Dyer, A.R. , Daviglus, M.L.. Costa, P.T., \& Stamler, J. (1991). Neuroticism and cynicism and risk of death in middle-aged men: The Western Electric Study. Psychosomatic Medicine, 53, 165 175

Alonzo, A., Simon, A., \& Feinleib, M. (1975). Prodromala of myocardial infarction and sudden death. Circulation, 52, 1056-1062.

American Psychiatric Association (1994). Diagnostic and statistical manual of mental disorders, fourth edition. Washington: American Psychiatric Association.

Appels, A. (1980). Psychological prodromata of myocardial infarction and sudden death. Psychotherapy and Psychosomatics, 34, 187-195.

Appels, A., Falger, P.R.J., \& Schouten, E.G.W. (1993). Vital exhaustion as risk indicator for myocardial infarction in women. Journal of Psychosomatic Research, $37,881-890$.

Appels, A., Höppener, P., \& Mulder, P. (1987). A questionnaire to assess premonitory symptoms of myocardial infarction. International Journal of Cardiology, 17 , 15-24.

Appels, A., Jenkins, C.D., \& Rosenman, R.H. (1982). Coronary-prone behavior in the Netherlands: A cross-cultural validation study. Journal of Behavioral Medicine, 5, 83-90.

Appels, A., Kop, W., Meesters, C., Markusse, R., Golombeck, B., \& Falger, P. (1994). Vital exhaustion and the acute coronary syndromes. In S. Maes, H. LeventhaI, \& M. Johnston (Eds.), International Review of Health Psychology, Volume 3 (pp. 65-95). New York: Wiley.

Appels, A., \& Mendes de Leon, C.F. (1989). The association between vital exhaustion, unstable angina, and future myocardial infarction. In Th. von Arnim \& A. Maseri (Eds.), Predisposing conditions for acute ischemic syndromes. Darmstadt: Steinkopff Verlag.

Appels, A., \& Mulder, P. (1988). Excess fatigue as a precursor of myocardial infarction. European Heart Journal, 9, 758-764.

Appels, A., \& Mulder, P. (1989). Fatigue and heart disease. The association between "vital exhaustion" and past, present and future coronary heart disease. Joumal of Psychosomatic Research, 33, 727-738. 
Appels, A., Mulder, P., van 't Hof, M., Jenkins, C.D., van Houtem, J., \& Tan, F. (1987). A prospective study of the Jenkins Activity Survey as a risk indicator for coronary heart disease in the Netherlands. Journal of Chronic Diseases, 40, 959965.

Appels, A., \& Otten, F. (1992). Exhaustion as precursor of cardiac death. British Journal of Clinical Psychology, 31, 351-356.

Appels, A., \& Schouten, E. (1991). Waking up exhausted as a risk indicator of myocardial infarction. American Journal of Cardiology, 68, 395-398.

Arlow, J.A. (1945). Identification mechanisms in coronary occlusion. Psychosomatic Medicine, 7, 195-210.

Arrindell, W.A., Emmelkamp, P.M.G., Brilman, E., \& Monsma, A. (1983). Psychometric evaluation of an inventory for assessment of parental rearing practices. A Dutch form of the EMBU. Acta Psychiatrica Scandinavica, 67, 163-177.

Arrindell, W.A., \& Ende, J. van der (1984). Replicability and invariance of dimensions of parental rearing behaviour: Further Dutch experiences with the EMBU. Personality and Individual Differences, 5, 671-682.

Arrindell, W.A., Perris, C., Eisemann, M., Perris, H., van der Ende, J., Ross, M., Benjaminsen, S., Gaszner, P., \& del Vecchio, M. (1986). Cross-national generalizability of patterns of parental rearing behaviour: invariance of EMBU dimensional representations of healthy subjects from Australia, Denmark, Hungary, Italy and The Netherlands. Personality and Individual Differences, 7, 103-112.

Arrowood, M., Uhrich, K., Gomillion, C., Popio, K., \& Raft, D. (1982). New markers of coronary-prone behavior in a rural population. Psychosomatic Medicine, 44, 119. (Abstract)

Averill, J.R. (1982). Anger and aggression: An essay on emotion. New York: Sprirger-Verlag.

Bandura, A. (1973). Aggression: A social learning analysis. Englewood Cliffs, NJ: Prentice-Hall.

Bandura, A. (1977). Social learning theory. Englewood Cliffs, NJ: Prentice-Hall.

Barefoot, J.C. (1992). Developments in the measurement of hostility. In H.S. Friedman (Ed.), Hostility, coping, and health (pp. 13-31). Washington, DC: American Psychological Association.

Barefoot, J.C., Dahlstrom, W.G., \& Williams, R.B. (1983). Hostility, CHD incidence, and total mortality: A 25-year follow-up study of 255 physicians. Psychosomatic Medicine, 45, 59-63.

Barefoot, J.C., Dodge, K.A., Peterson, B.L., Dahlstrom, W.G., \& Williams, R.B. (1989). The Cook-Medley Hostility Scale: Item content and ability to predict survival. Psychosomatic Medicine, 51, 46-57.

Barefoot, J.C., Haney, T.L., Harper, R.R., Chesney, M.A., Siegler, I.C., \& Williams, R.B. (1992). Interview assessed hostility and coronary artery disease in angiography patients. Manuscript submitted for publication. 
Barefoot, J.C., \& Lipkus, I.M. (1994). The assessment of anger and hostility. In A.W. Siegman \& T.W. Smith (Eds.), Anger, hostility, and the heart (pp. 43-66). Hillsdale, NJ: Lawrence Erlbaum Associates.

Barefoot, J.C., Patterson, J.C., Haney, T.L., Cayton, T.G., Hickman, J.R., \& Williams, R.B. (1994). Hostility in asymptomatic men with angiographically confirmed coronary artery disease. American Journal of Cardiology, 74, 439-442. Barefoot, J.C., Peterson, B.L., Dahlstrom, W.G., Siegler, I.C., Anderson, N.B., \& Williams, R.B. (1991). Hostility patterns and health implications: Correlates of Cook-Medley Hostility Scale scores in a national survey. Health Psychology, I0, 18-24.

Barefoot, J.C., Siegler, I.C., Nowlin, J.B., Peterson, B.L., Haney, T.L., \& Williams, R.B. (1987). Suspiciousness, health, and mortality: A follow-up study of 500 older adults. Psychosomatic Medicine, 49, 450-457.

Barefoot, J.C., Williams, R.B., Dahlstrom, W.G., \& Dodge, K.A. (1987). Predicting mortality from scores on the Cook-Medley hostility scale: A follow-up study of 118 lawyers. Psychosomatic Medicine, 49, 210.

Bendig, A.W. (1962). Factor analytic scales of covert and overt hostility. Joumal of Consulting Psychology, 26, 200.

Bendig, A.W. (1956). The development of a short form of the manifest anxiety scale. Journal of Consulting Psychology, 20, 384.

Bengtsson, C., Hällström, T., \& Tibblin, G. (1973). Social factors, stress experience, and personality traits in women with ischemic heart disease, compared to a population sample of women. Acta Medica Scandinavica, 549(Suppl.), 82-92.

Biaggio, M.K., Supplee, K., \& Curtis, N. (1981). Reliability and validity of four anger scales. Joumal of Personality Assessment, 45, 639-648.

Blumenthal, J.A., Barefoot, J., Burg, M.M., \& Williams, R.B. (1987). Psychological correlates of hostility among patients undergoing coronary angiography. British Journal of Medical Psychology, 60, 349-355.

Blumenthal, J.A., Williams, R.B., Kong, Y., Schanberg, S.M., \& Thompson, L. W. (1978). Type A behavior pattern and coronary atherosclerosis. Circulation, 58 , 634-639.

Brandenburg, R.O., Fuster, V., Giuliani, E.R., \& MoGoon, D.C. (Eds.) (1987). Cardiology: fundamentals and practice. Chicago/London: Yearbook Medical Publishers.

Braunwald, E. (Ed.) (1988). Heart Disease. Philadelphia: W.B. Saunders.

Bushnan, B.J., Cooper, H.M., \& Lemke, K.M. (1991). Meta-analysis of factor analyses: An illustration using the Buss-Durkee Hostility Inventory. Personality and Social Psychology Bulletin, 17, 344-349.

Buss, A.H. (1961). The psychology of aggression. New York: Wiley.

Buss, A.H., \& Durkee, A. (1957). An inventory for assessing different kinds of hostility. Journal of Consulting Psychology, 21, 343-349. 
Buss, A.H., \& Perry, M. (1992). The aggression questionnaire. Journal of Personality and Social Psychology, 63, 452-459.

Caffrey, B. (1968). Reliability and validity of personality and behavioral measures in a study of coronary heart disease. Journal of Chronic Diseases, 21, 191-204.

Campbell, D.T., \& Stanley, J.C. (1963). Experimental and quasi-experimental designs for research. Chicago: Rand McNally.

Carmelli, D., Rosenman, R.H., \& Swan, G.E. (1988). The Cook and Medley HO scale: A heritability analysis in adult male twins. Psychomatic Medicine, 50, 165 174.

Carney, R., Rich, M., Freedland, K., Saini, J., te Velde, A., Simeone, C., \& Clark, K. (1988). Major depressive disorder predicts cardiac events in patients with coronary heart disease. Psychosomatic Medicine, 50, 627-633.

Carver, C.S. (1989). How should multifaceted personality constructs be tested? Issues illustrated by self-monitoring, attributional style, and hardiness. Journal of Personality and Social Psychology, 56, 577-585.

Case, R.B., Heller, S.S., Case, N.B., \& Moss, A.J. (1985). Type A behavior and survival after acute myocardial infarction. New England Journal of Medicine, 312 , $737-741$.

Chesney, M.A., Hecker, M.H.L., \& Black, G.W. (1988). Coronary-prone components of Type A behavior in the WCGS: A new methodology. In B.K. Houston \& C.R. Snyder (Eds.), Type A behavior pattem: Research, theory, and intervention (pp. 168-188). New York: Wiley.

Cohen, J. (1960). A coefficient of agreement for nominal scales. Educational Psychology Measures, 20, 37-46.

Cook, W.W., \& Medley, D.M. (1954). Proposed hostility and pharisaic-virtue scales for the MMPI. Journal of Applied Psychology, 38, 414-418.

Cooper, T., Detre, T., \& Weiss, S.M. (1981). Coronary-prone behavior and coronary heart disease: A critical review. Circulation, 63, 1199-1215.

Costa, P.T., \& McCrae, R.R. (1985a). The Neo Personality Inventory Manual. Odessa, FL: Psychological Assessment Resources.

Costa, P.T., \& McCrae, R.R. (1985b). Hypochondriasis, neuroticism, and aging: When are somatic complaints unfounded? American Psychologist, 40, 19-28.

Costa, P.T., \& McCrae, R.R. (1987). Neuroticism, somatic complaints, and disease: Is the bark worse than the bite? Journal of Personality, 55, 299-316.

Costa, P.T., McCrae, R.R., \& Dembroski, T.M. (1989). Agreeableness versus antagonism: Explication of a potential risk factor for CHD. In A.W. Siegman \& T.M. Dembroski (Eds.), In search of coronary-prone behavior: Beyond Type A (pp. 4163). Hillsdale, NJ: Lawrence Erlbaum Associates.

Costa, P.T., Zonderman, A.B., Engel, B.T., Baile, W.F., Brimlow, B.L., \& Brinker, J. (1985). The relation of chest pain symptoms to angiographic findings of coronary artery stenosis and neuroticism. Psychosomatic Medicine, 47, 285-293. 
Costa, P.T., Zonderman, A.B., McCrae, R.R., \& Willams, R.B. (1986). Cynicism and paranoid alienation in the Cook and Medley Ho scale. Psychosomatic Medicine, $48,283-285$.

Croog, S.H., Koslowsky, J., \& Levine, S. (1976). Personallity self-perception of male heart patients and their wives: Issues of congruence and "coronary personality". Perceptual and Motor Skills, 43, 927-937.

Croyle, R.T., \& Sande, G.N. (1988). Denial and confirmatory search: paradoxical consequences of medical diagnosis. Journal of Applied Social Psychology, 18, 473 490.

Darley, J.M. , \& Fazio, R.H. (1980). Expectancy confirmation processes arising in the social interaction sequence. American Psychologist, 35, 867-881.

Dembroski, T.M., \& Costa, P.T. (1987). Coronary-prone behavior: Components of the Type A pattern and hostility. Journal of Personality, 55, 211-235.

Dembroski, T.M., \& MacDougall, J.M. (1983). Behavioral and psychophysiological perspectives on coronary-prone behavior. In T.M. Dembroski, T.M. Schmidt, \& G. Blümchen (Eds.), Biobehavioral bases of coronary heart disease. Basel: Karger.

Dembroski, T.M., MacDougall, J.M., Costa, P.T., \& Grandits, G.A. (1989). Components of hostility as predictors of sudden death and myocardial infarction in the Multiple Risk Factor Intervention Trial. Psychosomatic Medicine, 51, 514-522.

Dembroski, T.M., MacDougall, J.M., Williams, R.B., Haney, T.L., \& Blumenthal, J.A. (1985). Components of Type A, hostility, and anger-in: Relationship to angiographic findings. Psychosomatic Medicine, 47, 219-233.

Diamond, E.L. (1982). The role of anger and hostility in essentiall hypertension and coronary heart disease. Psychological Bulletin, 92, 410-433.

Diest, R. van, \& Appels, A. (1991). Vital exhaustion and depression: a conceptual study. Journal of Psychosomatic Research, 35, 535-544.

Diest, R. van, \& Appels, A. (1994). Sleep physiological characteristics of exhausted men. Psychosomatic Medicine, 56, 28-35.

Dijl, H. van (1982). Myocardial infarction patients and heightened aggressiveness/hostility. Journal of Psychosomatic Research, 26, 203-208.

Dimsdale, J.F., Hackett, T.P., \& Hutter, A.M. (1979). Type A behavior and angiographic findings. Journal of Psychosomatic Research, 23, 273-276.

Dunbar, H.F. (1943). Psychosomatic diagnosis. New York: Paul Hoeber.

Edmunds, G., \& Kendrick, D.C. (1980). The measurement of human aggressiveness. West Sussex, England: Ellis Horwood.

Emmelkamp, P.M.G., \& Karsdorp, E.P. (1987). The effects of perceived parental rearing style on the development of Type A pattern. European Joumal of Personality, $1,223-230$.

Falger, P.R.J. (1989). Life-span development and myocardial infarction: an epidemiological study. PhD thesis. Maastricht: University of Limburg. The Netherlands. 
Falger, P.R.J., \& Schouten, E.G.W. (1992). Exhaustion, psychological stressors in the work environment, and acute myocardial infarction in adult men. Joumal of Psychosomatic Research, 36, 777-786.

Fontana, A.F., Kerns, R.D., Blatt, S.J., Rosenberg, R.L., Burg, M.M., \& Colonese, K.L. (1989). Cynical mistrust and the search for self-worth. Joumal of Psychosomatic Research, 33, 449-456.

Frank, K.A., Heller, S.S., Kornfeld, D.S., Sporn, A.A., \& Weiss, M.B. (1978). Type A behavior pattern and coronary angiographic findings. Joumal of the American Medical Association, 240, 761-763.

Fraser, G. (1978). Sudden death in Auckland. Australian and New Zealand Journal of Medicine, 8, 490-499.

Friedman, H.S., \& Booth-Kewley, S. (1987). Personality, Type A behavior, and coronary heart disease: The role of emotional expression. Journal of Personality and Social Psychology, 53, 783-792.

Friedman, M. \& Rosenman, R.H. (1974). Type A behavior and your heart. New York: Knopf.

Friedman, M. Rosenman, R.H., Straus, R., Wurm, M., \& Kositchek, R. (1968). The relationship of behavior pattern to the state of the coronary vasculature. A study of 51 autopsy subjects. American Journal of Medicine, 44, 525-538.

Friedman, M., Thoresen, C.E., Gill, J.J., Ulmer, D., Powell, L.H., Price, V.A., Brown, B., Thompson, L., Rabin, D.D., Breall, W.S., Bourg, E., Levy, R., \& Dixon, T. (1986). Alteration of Type A behavior and its effect on cardiac recurrences in post myocardial infarction patients: Summary results of the recurrent coronary prevention project. American Heart Journal, 112, 653-665.

Fuster, V., Badimon, L., Badimon, J.J., \& Chesebro, J.H. (1992). The pathogenesis of coronary artery disease and the acute coronary syndromes. New England Journal of Medicine, $326,242-250,310-318$.

Gillum, R., Feinleib, M., Margolis, J.R. Fabsitz, R., \& Brasch, R. (1976). The pre-hospital phase of acute myocardial infarction and sudden death. Preventive Medicine, 5, 408-413.

Golombiewski, R.T., Munzenrider, R.F., \& Stevenson, J.G. (1986). Stress in organizations: Toward a phase model of burnout. New York: Praeger.

Greenglass, E.R., \& Julkunen, J. (1989). Construct validity and sex differences in Cook-Medley hostility. Personality and Individual Differences, 10, 209-218.

Hăllström, T., Lapidus, L., Bengtsson, C., \& Edström, K. (1986). Psychosocial factors and risk of ischaemic heart disease and death in women: A twelve-year follow-up of participants in the population study of women in Gothenburg. Sweden. Jourmal of Psychosomatic Research, 30, 451-459.

Hardy, J.D., \& Smith, T.W. (1988). Cynical hostility and vulnerability to disease: Social support, life stress, and physiological response to conflict. Health Psychology, 7, 447-459. 
Hayano, J., Takeuchi, S., Yoshida, S., Jozuka, H., Mishima, N., \& Fujinami, T. (1989). Type A behavior pattern in Japanese employees: Cross-cultural comparison of major factors in Jenkins Activity Survey (JAS) responses. Journal of Behavional Medicine, 12, 219-231.

Haynes, S.G., Feinleib, M., \& Kannel, W.B. (1980). The relationship of psychosocial factors to coronary heart disease in the Framingham study: III. Eight-year incidence of coronary heart disease. American Joumal of Epidemiology, 111, 3758.

Hearn, M.D., Murray, D.M., \& Luepker, R.V. (1989). Hostility, coronary heart disease, and total mortality: A 33-year follow-up study of university students. Jounnal of Behavioral Medicine, 12, 105-121.

Hecker, M.H.L., Chesney, M.A., Black, G.W. \& Frautschi, N. (1988), Coronaryprone behaviors in the Western Collaborative Group Study. Psychosomatic Medicine, 50, 153-164.

Helmer, D.C., Ragland, D.R., \& Syme, S.L. (1991). Hostility and coronary artery disease. American Journal of Epidemiology, 133, 112-122.

Helmers, K.F., Krantz, D.S., Howell, R.H., Klein, J., Bairey, C.N., \& Rozanski, A. (1993). Hostility and myocardial ischemia in coronary artery disease patients: Evaluation by gender and ischemic index. Psychosomatic Medicine, 55, 29-36.

Helmers, K.F., Posluszny, D.M., \& Krantz, D.S. (1994). Associations of hostility and coronary artery disease: A review of studies. In A.W. Siegman \& T.W. Smith (Eds.), Anger, hostility, and the heart (pp. 67-96). Hillsdale, NJ: Lawrence Erlbaum Associates.

Holroyd, K.A., \& Coyne, J. (1987). Personality and health in the 1980s: Psychosomatic medicine revisited? Journal of Personality, 55, 359-375.

Houston, B.K. (1994). Anger, hostility, and psychophysiological reactivity. In A.W. Siegman \& T.W. Smith (Eds.), Anger, hostility, and the heart (pp. 97-115). Hillsdale, NJ: Lawrence Erlbaum Associates.

Houston, B.K., \& Kelly, K.E. (1989). Hostility in employed women: Relation to work and marital experiences, social support, stress, and anger expression. Personality and Social Psychology Bulletin, 15, 175-182.

Houston, B.K., \& Vavak, C.R. (1991). Cynical hostility: Developmental factors, psychosocial correlates, and health behaviors. Health Psychology, 10, 9-17.

Hur, Y.M., \& Bouchard, T.J. (1995). Genetic influences on perceptions of childhood family environment: A reared apart twin study. Child Development, 66, 330-345. Hurst, J.W., Schlant, R.C., Rackley, C.E., Sonnenblick, E.H., \& Wenger, N.K. (Eds.) (1990). The Heart. New York: McGraw-Hill.

Insull, W. (1973). Coronary risk handbook. New York: American Heart Association. lzard, C.E. (1979). Human emotions. New York: Plenum.

Jenkins, C.D., Rosenman, R.H., \& Friedman, M. (1968). Replicability of rating the coronary-prone behavior pattern. British Journal of Preventive and Social Medicine, 22, 16-22. 
Jenkins, C.D., Zyzanski, S.J., \& Rosenman, R.H. (1978). Coronary-prone behavior: One pattern or several? Psychosomatic Medicine, 40, 25-43.

Jenkins, C.D., Zyzanski, S.J., \& Rosenman, R.H. (1979). Jenkins Activity Survey Manual. New York: Psychological Corporation.

Joesoef, M.R., Wetterhall, S.F., DeStefano, F., Stroup, N.E., \& Fronek, A. (1989). The association of peripheral arterial disease with hostility in a young, healthy veteran population. Psychosomatic Medicine, 51, 285-289.

Johnston, D.W., Cook, D.G., \& Shaper, A.G. (1987). Type A behaviour and ischemic heart disease in middle aged British men. British Medical Journal, 295, 86-89.

Kannel, W.B., Neaton, D.J., \& Wentworth, D. (1986). Overall and coronary heart disease mortality rates in relation to major risk factors in 325,348 men screened for the MRFIT. American Heart Journal, 112, 825-836.

Kannel, W.B., \& Thom, T.J. (1994). Incidence, prevalence, and mortality of cardiovascular diseases. In R.C. Schlant, R.W. Alexander, R.A. O'Rourke, R. Roberts, \& E.H. Sonnenblick (Eds.), The heart: Arteries and veins (pp. 185-197). New York: McGraw-Hill.

Kaplan, J.R., Botchin, M.B., \& Manuck, S.B. (1994). Animal models of aggression and cardiovascular disease. In A.W. Siegman \& T.W. Smith (Eds.), Anger, hostility, and the heart (pp. 127-148). Hillsdale, NJ: Lawrence Erlbaum Associates.

Keith, R.A., Lown, B., \& Stare, F.J. (1965). Coronary heart disease and behavior patterns: An examination of method. Psychosomatic Medicine, 27, 424-434.

Kemple, C. (1945). Rorschach method and psychosomatic diagnosis: Personality traits of patients with rheumatic disease, hypertensive cardiovascular disease, coronary occlusion, and fracture. Psychosomatic Medicine, 7, 85-89.

Keys, A. (1970). Coronary heart disease in seven countries: XIII multiple variables. Circulation, 41, 138-144.

Kinlen, L. (1973). Incidence and presentation of myocardial infarction in an English community. British Heart Journal, 35, 616-622.

Klacboe, G., Otterstad, J.E., Winsnes, T., \& Espeland, N. (1987). Predictive value off prodromal symptoms in myocardial infarction. Acta Medica Scandinavica, 222, $27-30$.

Kneip, R.C., Delamater, A.M., Ismond, T., Milford, C., Salvia, L., \& Schwartz, D. (1993). Self- and spouse ratings of anger and hostility as predictors of coronary heart disease. Health Psychology, 12, 301-307.

Kop, W.J. (1994). The predictive value of vital exhaustion in the clinical course after coronary angioplasty. PhD thesis. Maastricht: University of Limburg. The Netherlands.

Kop, W.J., Appels, A.P.W.M., Mendes de Leon, C.F., de Swart, H.B., \& Bär, F.W. (1994). Vital exhaustion predicts new cardiac events after successful coronary angioplasty. Psychosomatic Medicine, 56, 281-287.

Kopec, J.A., \& Esdaile, J.M. (1990). Bias in case-control studies. A review. Joumal of Epidemiology and Community Health, 44, 179-186. 
Koskenvuo, M., Kaprio, J., Rose, R.J., Kesaniemi, A., Sarna, S., Heikkila, K., \& Langinvainio, H. (1988). Hostility as a risk factor for mortality and ischemic heart disease in men. Psychosomatic Medicine, 50, 330-340.

Krantz, D.S., Contrada, R.J., Hill, R.O., \& Friedler, E. (1988). Environmental stress and biobehavioral antecedents of coronary heart disease. Journal of Consuling and Clinical Psychology, 56, 333-341.

Krantz, D.S., \& Durel, L.A. (1983). Psychobiological substrates of the Type A behavior pattern. Health Psychology, 2, 393-411.

Krantz, D.S., \& Manuck, S.B. (1984). Acute psychophysiologic reactivity and risk of cardiovascular disease: A review and methodological critique. Psychological Bulletin, 96, 535-564.

Krantz, D.S., Sanmarco, M.I., Selvester, R.H., \& Matthews, K.A. (1979). Psychological correlates of progression of atheroscierosis in men. Psychosomatic Medicine, 41, 467-476.

Kuller, L.H. (1978). Prodromata of sudden death and myocardial infarction. Advances in Cardiology, 25, 61-72.

Kuller, L., Cooper, M., \& Perper, J. (1972). Epidemiology of sudden death. Archives of Internal Medicine, 129, 714-719.

Ladwig, K.H., Kieser, M., Konog, J., Breithardt, G., \& Borggrefe, M. (1991). Affective disorders and survival after acute myocardial infarction. Results from the postinfarction late-potential study. European Heart Joumal, 12, 959-964.

Lange, A., Pahlich, A., Sarucco, M., Smits, G., Dehghani, B., \& Hanewald, G. (1995). Psychometric characteristics and validity of the Dutch adaptation of the Buss-Durkee Hostility Inventory (The BDHI-D). Behaviour Research and Therapy, $33,223-227$.

Lee, D., Mendes de Leon, C.F., Jenkins, C.D., Croog, S.H., Levine, S., \& Sudilovsky, A. (1992). Relation of hostility to medication adherence, symptom complaints, and blood pressure reduction in a clinical field trial of antihypertensive medication. Journal of Psychosomatic Research, 36, 181-190.

Leibowitz, J.O. (1970). The history of coronary heart disease. Berkeley: University of California Press.

Leiker, M., \& Hailey, B.J. (1988). A link between hostility and disease: Poor health habits? Behavioral Medicine, 3, 129-133.

Leiter, M.P. (1993). Burnout as a developmental process: Consideration of models. In W.B. Schaufeli, C. Maslach, \& T. Marek (Eds.), Professional Burnout: Recent developments in theory and research. Washington: Taylor \& Francis.

Leon, G.R., Finn, S.E., Murray, D., \& Bailey, J.M. (1988). Inability to predict cardiovascular disease from hostility scores or MMPI items related to Type A behavior. Journal of Consulting and Clinical Psychology, 56, 597-600.

Lipkus, I.M., Barefoot, J.C., Beckham, J.C., \& Haney, T.L. (1993, March). The structure of the Cook-Medley hostility scale as assessed by multidimensional scaling. Paper presented at the meetings of the Society of Behavioral Medicine, San Francisco. 
MacDougall, J.M., Dembroski, T.M., Dimsdale, J.E., \& Hackett, T. (1985). Components of Type $\mathrm{A}$, hostility, and anger-in: Further relationships to angiographic findings. Health Psychology, 4, 137-152.

Manuck, S.B., Kaplan, J.R., Muldoon, M.F., Adams, M.R., \& Clarkson, T.B. (1991). The behavioral exacerbation of atherosclerosis and its inhibition by propra nolol. In P.M. McCabe, N. Schneiderman, T.M. Field, \& J.S. Skyler (Eds.), Stress, coping, and disease (pp. 51-72). Hillsdale, NJ: Lawrence Erlbaum Associates.

Maslach, C., \& Jackson, S.E. (1986). Maslach Burnout Inventory. Palo Alto: Consulting Psychologists Press.

Matthews, K.A. (1985). Assessment of Type A behavior, anger, and hostility in epidemiological studies of cardiovascular disease. In A.M. Ostfeld \& E.D. Eaker (Eds.), Measuring psychosocial variables in epidemiologic studies of cardiovascular disease (NIH Publication No. 85-2270, 153-184). Washington, DC: U.S. Department of Health and Human Services.

Matthews, K.A. (1988). Coronary heart disease and Type A behaviors: Update on and alternative to the Booth-Kewley and Friedman (1987) quantitative review. Psychological Bulletin, 104, 373-380.

Matthews, K.A., Glass, D.C., Rosenman, R.H., \& Bortner, R.W. (1977). Competitive drive, pattern A, and coronary heart disease: A further analysis of some data from the Western Collaborative Group Study. Journal of Chronic Diseases, 30 , 489-498.

Matthews, K.A., Jamison, J.W., \& Cottington, E.M. (1985). Assessment of Type A, anger, and hostility: A review of scales through 1982. In A.M. Ostfeld \& E.D. Eaker, (Eds.), Measuring psychosocial variables in epidemiologic studies of cardiovascular disease (NIH Publication No. 85-2270, pp. 207-312). Washington, DC: U.S. Department of Health and Human Services.

Matthews, K.A., Rosenman, R.H., Dembroski, T.M., Harris, E.L., \& MacDougall, J.M. (1984). Familial resemblance in components of the Type A behavior pattern: A reanalysis of the California Type A Twin Study. Psychosomatic Medicine, 46, $512-522$

Matthews, K.A., \& Woodall, K.L. (1988). Childhood origins of overt Type A behaviors and cardiovascular reactivity to behavioral stressors. Annals of Behavioral Medicine, 10, 71-77.

Matthews, K.A., Woodall, K.L., Engebretson, T.O., McCann, B.S., Stoney, C.M., Manuck, S.B., \& Saab, P.G. (1992). Influence of age, sex, and family on Type A and hostile attitudes and behaviors. Healt Psychology, 11, 317-323.

McCranie, E.W., Watkins, L.O., Brandsma, J.M., \& Sisson, B.D. (1986). Hostility, coronary heart disease (CHD), and total mortality: Lack of association in a 25 year follow-up study of 478 physicians. Journal of Behavioral Medicine, 9, 119 125. 
McGonigle, M.M., Smith, T.W., Berjamin, L.S., \& Tumer, C.W. (1993). Hostility and nonshared family environment: A study of monozygotic twins. Journal of Research in Personality, 27, 23-34.

Meesters, C., \& Appels, A. (1995a). An interview to measure vital exhaustion. I. Development and comparison with the Mastricht Questionnaire. Psychology and Health, in press.

Meesters, C., \& Appels, A. (1995b). An interwiew to measure vital exhaustion. II. Reliability and validity of the interview and correlations of vital exhaustion with personality characteristics. Psychology and Health, in press.

Meesters, C., \& Muris, P. (1995). The relationship between hostility and perceived parental rearing behaviour: A study of male myocardial infarction patients and healthy controls (submitted).

Meesters, C.M.G., Muris, P., \& Backus, I.P.G. (1995). Dimensions of hostitity and myocardial infarction in adult males. Joumat of Psychosomatic Research, in press.

Meesters, C., Muris, P., \& Esselink, T. (1995). Hostility and perceived parental rearing behaviour. Personality and Individual Differences, 18, 567-570.

Meesters, C.M.G., \& Smulders, I. (1994). Hostility and myocardial infarction in men. Joumal of Psychosomatic Research, 38, 727-734.

Megargee, E.I. (1985). The dynamics of aggression and their application to cardiovascular disorders. In M.A. Chesney \& R.H. Rosenman (Eds.), Anger and hostility in cardiovascular and behavioral disorders (pp. 31-57). Washington, DC: Hemisphere.

Mendes de Leon, C.F. (1988). Behavioral and emotional precursors of acute heart disease. PhD thesis. Galveston: Unversity of Texas Medical Branch, USA.

Mendes de Leon, C.F. (1992). Anger and impatience/irritability in patients of low socioeconomic status with acute coronary heart disease. Joumal of Behavioral Medicine, 15, 273-284.

Menninger, K.A., \& Menninger, W.C. (1936). Psychoanalytic observations in cardiac disorders. American Heart Joumal, 11, 10-21.

Miettinen, O. (1985). The "case-control" study: Valid selection of subjects. Journal of Chronic Diseases, 38, 543-548.

Miles, H., Waldfoger, S., Barrabee, E. \& Cobb, S. (1954). Psychosomatic study of 46 young men with coronary artery disease. Psychosomatic Medicine, 16, 455477.

Miller, C.K. (1965). Psychological correlates of coronary artery disease. Psychosomatic Medicine, 27, 257-265.

Miller, T.Q., Turner, C.W., Tindale, R.S., Posavac, E.J., \& Dugoni, B.L. (1991). Reasons for the trend toward null findings in research on Type A behavior. Psychological Bulletin, 110, 469-485.

Musante, L., MacDougall, J.M., Dembroski, T.M., \& Costa, P.T. (1989). Potential for hostility and dimensions of anger. Health Psychology, 8, 343-354. 
Neissen, 1. (1993). Met hart en ziel: Een studie naar de validiteit en betrouwbaarheid van de Cook-Medley Hostility Scale en de Buss-Durkee Hostility Inventory. Unpublished master's thesis. Maastricht: University of Limburg.

Nelissen-de Vos, Y.C.M. (1994). Sleep problems preceding myocardial infarction. PhD thesis. Maastricht: University of Limburg., The Netherlands.

Norusis, M.J. (1990). SPSS/PC+ 4.0. Chicago: SPSS Inc.

Ostield, A.M., Lebovits, B.Z., Shekelle, R.B., \& Paul, O. (1964). A prospective study of the relationship between personality and coronary heart disease. Journal of Chronic Diseases, 17, 265-276.

Pedersen, N.L., Lichtenstein, P., Plomin, R., DeFaire, U., McClearn, G.E., \& Matthews, K.A. (1989). Genetic and environmental influences for Type A-like measures and related traits: A study of twins reared apart and twins reared together. Psychosomatic Medicine, 51, 428-440.

Perris, C., Jacobsson, L. Lindström, H., Knorring, L. von, \& Perris, H. (1980). Development of a new inventory for assessing memories of parental belhaviour. Acta Psychiatrica Scandinavica, 61, 265-274.

Pickering, T.G. (1985). Should studies of patients undergoing coronary angiography be used to evaluate the role of behavioral risk factors for coronary heart disease? Journal of Behavioral Medicine, 8, 203-213.

Pines, A., \& Aronson, E. (1988). Career burnout: causes and cures. New York: The Free Press.

Plomin, R., \& Bergeman, C.S. (1991). The nature of nurture: Genetic influence on "environmental" measures. Behawioral and Brain Sciences, 14, 373-427.

Plomin, R., McClearn, G.E., Pedersen, N.L., Nesselroade, J.R., \& Bergeman, C.S. (1988). Genetic influence on childhood family environment perceived retrospectively from the last half of the life span. Developmental Psychology, 24, 738745.

Pope, M.K., Smith, T.W., \& Rhodewalt, F. (1990). Cognitive, behavioral, and affective correlates of the Cook and Medley Hostility Scale. Journal of Personality Assessment, 54, 501-51.4.

Powell, L.H., \& Thoresen, C.E. (1985). Behavioral and physiologic determinants of long-term prognosis after myocardial infarction. Journal of Chronic Diseases, 38 , $253-263$.

Ragland, D.R., \& Brand, R.J. (1988a). Coronary heart disease mortality in the Western Collaborative Group Study: Follow-up experience of 22 years. American Journal of Epidemiology, 127, 462-475.

Ragland, D.R.; \& Brand, R.J. (1988b). Type A behavior and mortality from coronary heart disease. New England Journal of Medicine, 318, 65-69.

Ranchor, A.V. (1994). Social class, psychosocial factors and disease. From description to explanation. PhD thesis. Groningen: University of Groningen, The Netherlands. 
Rissanen, V., Romo, M., \& Siltanen, P. (1978). Premonitory symptoms and stress factors preceding sudden death from ischaemic heart disease. Acta Medica Scandinavica, 204, 389-396.

Robins, L.N., Schoenberg, S.P., Holmes, S.J., Ratcliff, K.S., Benham, A., \& Works, J. (1985). Early home environment and retrospective recall: A test for concordance between siblings with and without psychiatric disorders. American Journal of Orthopsychiatry, 55, 27-41.

Rose, R.J. (1988). Genetic and environmental variance in content dimensions of the MMPI. Journal of Personality and Social Psychology, 55, 302-311.

Rose, G.A., Blackburn, H., Gillum, R.F., \& Prineas, R.J. (1982). Cardiovascular Survey Methods (Second edition). Geneva: World Health Organization.

Rosenman, R.H. (1978). The interview method of assessment of the coronary-prone behavior pattern. In T.M. Dembroski, S.M. Weiss, J.L. Shields, S.G. Haynes, \& M. Feinleib (Eds.), Coronary-prone behavior (pp. 55-69). New York: SpringerVerlag.

Rosenman, R.H. (1983). Current status of risk factors and Type A behavior pattern in the pathogenesis of ischemic heart disease. In T.M. Dembroski, T.H. Schmidt, \& G. Blümchen (Eds.), Biobehavioral bases of coronary heart disease. New York: Karger.

Rosenman, R.H., Brand, R.J., Jenkins, C.D., Friedman, M., Straus, R., \& Wurm, M. (1975). Coronary heart disease in the Western Collaborative Group Study. Final follow-up experience of $81 / 2$ years. Journal of the American Medical Association, 233, 872-877.

Rosenman, R.H., \& Chesney, M.A. (1982). Stress, Type A behavior, and coronary disease. In L. Goldberger \& S. Breznitz (Eds.), Handbook of stress: Theoretical and clinical aspects. New York: Free Press.

Rosenman, R.H., \& Friedman, M. (1974). Neurogenic factors in pathogenesis of coronary heart disease. Medical Clinics of North America, 58, 269-279.

Rosenman, R.H., Swan, G.E., \& Carmelli, D. (1988). Definition, assessment, and evolution of the Type A behavior pattern. In B.K. Houston \& C.R. Snyder (Eds.), Type A behavior pattern: Research, theory, and intervention (pp. 8-31). New York: Wiley.

Ross, R. (1993). The pathogenesis of atherosclerosis: a perspective for the 1990"s. Nature, 362, 801-809.

Rothman, K.J. (1986). Modern epidemiology. Boston/Toronto: Little, Brown and Company.

Ryu, J.E., Thompson, C.J., \& Crouse, J.R. (1989). Selection of neighborhood controls for a study of coronary artery disease. American Journal of Epidemiology, $129,407-414$.

Sackett, D.L. (1979). Bias in analytic research. Journal of Chronic Diseases, 32, 5163. 
Sarason, I. (1961). Intercorrelations among measures of hostility. Journal of Clinical Psychology; 17, 192-195.

Scarr, S., \& McCartney, K. (1983). How people make their own environments: A theory of genotype $->$ environment effects. Child Development, 54, 424-435.

Scherwitz, L., Berton, K., \& Leventhal, H. (1977). Type A assessment and interaction in the behavior pattern interview. Psychosonatic Medicine, 39, 229-240.

Scherwitz, L., Perkins, L., Chesney, M., \& Hughes, G. (1991). Cook-Medley Hostility Scale and subsets: Relationship to demographic and psychosocial characteristics in young adults in the CARDIA study. Psychosomatic Medicine, 53 , 36-49.

Scherwitz, L.W., Perkins, L.L., Chesney, M.A., Hughes, G.H., Sidney, S., \& Manolio, T.A. (1992). Hostility and health behaviors in young adults: the CARDIA study. American Journal of Epidemiology, 136, 136-145.

Schlesselman, J.J. (1982). Case-control studies: Design, conduct, analysis. New York: Oxford University Press.

Schroeder, D.H., \& Costa, P.T. (1984). Influence of life event stress on physical illness: Substantive effects or methodological flaws? Journal of Personality and Social Psychology, 46, 853-863.

Selye, H. (1967). Stress in Health and Disease. Boston: Butterworth.

Selye, H. (1977). The stress of life. New York: McGraw-Hill.

Shekelle, R.B., Gale, M., \& Norusis, M. (1985). Type A score (Jenkins Activity Survey) and risk of recurrent coronary heart disease in the Aspirin Myocardial Infarction Study. American Joumal of Cardiology, 56, 221-225.

Shekelle, R.B., Gale, M. , Ostfeld, A.M., \& Paul, O. (1983). Hostility, risk of coronary heart disease, and mortality. Psychosomatic Medicine, 45, 109-114.

Shekelle, R.B., Hulley, S., Neaton, J., Billings, J., Borhani, N ., Gerace, T., Jacobs, D., Lasser, N. Mittlemark, M., Stamler, A., and the MRFIT Research Group. (1985). The MRFIT behavior pattern study. II. Type A behavior pattern and incidence of coronary heart disease. American Joumal of Epidemiology, 122, 559570.

Siegel, J.M. (1986). The Multidimensional Anger Inwentory. Journal of Personality and Social Psychology, 51, 191-200.

Siegler, I.C., Peterson, B.L. „Barefoot, J.C., \& Williams, R.B. (1992). Hostility during late adolescence predicts coronary risk factors at mid-life. American Journal of Epidemiology, 136, 146-154.

Siegler, I.C., Zonderman, A.B., Barefoot, J.C., Williams, R.B., Costa, P.T., \& McCrae, R.R. (1990). Predicting personality in adulthood from college MMPI scores: Implications for follow-up studies in psychosomatic medicine. Psychosomatic Medicine, 52, 644-652.

Siegman, A.W. (1994a). From Type A to hostility and anger: Reflections on the history of coronary-prone behavior. In A.W. Siegman \& T.W. Smith (Eds.), Anger, hostility, and the heart (pp. 1-21). Hillsdale, NJ: Lawrence Erlbaum Associates. 
Siegman, A.W. (1994b). Cardiovascular consequences of expressing and repressing anger. In A.W. Siegman \& T.W. Smith (Eds.), Anger, hostility, and the heart (pp. 173-197). Hillsdale, NJ: Lawrence Erlbaum Associates.

Siegman, A.W., Anderson, R.W., \& Berger, T. (1990). The angry voice: Its effects on the experience of anger and cardiovascular reactivity. Psychosomatic Medicine, $52,631-643$.

Siegman, A.W., Anderson, R., Herbst, J., Boyle, S., \& Wilkinson, J. (1992). Dimensions of anger-hostility and cardiovascular reactivity in provoked and angered men. Journal of Behavioral Medicine, 15, 257-272.

Siegman, A.W., \& Dembroski, T.M. (Eds.) (1989). In search of coronary-prone behavior: Beyond Type A. Hillsdale, NJ: Lawrence Erlbaum Associates.

Siegman, A.W., Dembroski, T.M., \& Ringel, N. (1987). Components of hostility and the severity of coronary artery disease. Psychosomatic Medicine, 49, 127-135.

Siegrist, J., Peter, R., Junge, A., Cremer, P., \& Seidel, D. (1990). Low status control, high effort at work and ischaemic heart disease: Prospective evidence from blue collar men. Social Science and Medicine, 31, 1127-1134.

Silverstone, P. (1987). Depression and outcome in acute myocardial infarction. British Medical Journal, 294, 219-220.

Simon, A., Feinleib, M., \& Thompson, H. (1972). Components of delay in the prehospital phase of acute myocardial infarction. American Journal of Cardiology, 30, 476-481.

Smith, T.W. (1992). Hostility and health: Current status of a psychosomatic hypothesis. Health Psychology, 11, 139-150.

Smith, T.W. (1994). Concepts and methods in the study of anger, hostility, and health. In A.W. Siegman \& T.W. Smith (Eds.), Anger, hostility, and the heart (pp. 23-42). Hillsdale, NJ: Lawrence Erlbaum Associates.

Smith, T.W., \& Christensen, A.J. (1992). Cardiovascular reactivity and interpersonal relations: Psychosomatic processes in social context. Journal of Social and Clinical Psychology, 11, 279-301.

Smith, T.W., \& Frohm, K.D. (1985). What's so unhealthy about hostility? Construct validity and psychosocial correlates of the Cook and Medley Ho scale. Health Psychology, 4, 503-520.

Smith, T.W., McGonigle, M., Turner, C.W., Ford, M.H., \& Slattery, M.L. (1991). Cynical hostility in adult male twins. Psychosomatic Medicine, 53, 684-692.

Smith, T.W., \& Pope, M.K. (1990). Cynical hostility as a health risk: Current status and future directions. Journal of Social Behavior and Personality, 5, 77-88.

Smith, T.W., Pope, M.K., Sanders, J.D., Allred, K.D., \& O'Keeffe, J.L. (1988). Cynical hostility at home and work: Psychosocial vulnerability across domains. Journal of Research in Personality, 22, 525-548.

Smith, T.W., Sanders, J.D., \& Alexander, J.F. (1990). What does the Cook and Medley Hostility Scale measure? Affect, behavior, and attributions in the marital context. Journal of Personality and Social Psychology, 58, 699-708. 
Smith, T.W., \& Williams, P.G. (1992). Personality and health: Advantages and limitations of the five-factor model. Joumal of Personality, $60,395-423$.

Snyder, M., \& Swann, W.B. (1978). Behavioral confirmation in social interaction: From social perception to social reality. Joumal of Experimental Social Psycholo$g y, 14,148-162$.

Spielberger, C.D., Jacobs, G.A., Russell, S.F., \& Crane, R.J. (1983). Assessment of anger: The state-trait anger scale. In J.N. Butcher \& C.D. Spielberger (Eds.), Advances in personality assessment, (Vol. 2). Hillsdale, NJ: Lawrence Erlbaum Associates.

Spielberger, C.D., Johnson, E.H., Russell, S.F., Crane, R.J., Jacobs, G.A., \& Worden, T.J. (1985). The experience and expression of anger: Construction and validation of an anger expression scale. In M.A. Chesney \& R.H. Rosenman (Eds.), Anger and hostility in cardiovascular and behavioral disorders (pp. 5-30). Washington, DC: Hemisphere.

Stevens, J.H., Turner, C.W., Rhodewalt, F., \& Talbot, S. (1984). The Type A behavior pattern and carotid artery atherosclerosis. Psychosomatic Medicine, 46, $105-113$.

Stoney, C.M., \& Engebretson, T.O. (1994). Anger and hostility: Potential mediators of the gender difference in coronary heart disease. In A.W. Siegman \& T.W. Smith (Eds.), Anger, hostility, and the heart (pp. 215-237). Hillsdale, NJ: Lawrence Erlbaum Associates.

Stowers, M., \& Short, D. (1970). Warning symptoms before myocardial infarction. British Heart Journal, 32, 833-838.

Suarez, E.C., \& Williams, R.B. (1990). The relationships between dimensions of hostility and cardiovascullar reactivity as a function of task characteristics. Psychosomatic Medicine, 52, 558-570.

Suls, J., \& Rittenhouse, J.D. (1990). Models to study linkages between personality and disease. In H.S. Friedman (Ed.), Personality and disease (pp. 38-64). New York: Wiley.

Suls, J., \& Sanders, G.S. (1989). Why do some behavioral styles place people at coronary risk? In A.W. Siegman \& T.M. Dembroski (Eds.), In search of coronary-prone behavior: Beyond Type A (pp. 1-20). Hillsdale, NJ: Lawrence Erlbaum Associates.

Suls, J., \& Wan, C.K. (1993). The relationship between trait hostility and cardiovascular reactivity: A quantitative review and analysis. $P_{S y c h o p h y s i o l o g y}, 30,615-$ 626.

Swan, G.E., Carmelli, D., \& Rosenman, R.H. (1990). Cook and Medley hostility and the Type A behavior pattern: Psychological correlates of two coronary-prone behaviors. Journal of Social Behavior and Personality, 5, 89-106.

Sykes, D.H., Haertel, U., Gostautas, A., \& Evans, A.E. (1992). The Framingham Type A behaviour pattern and coronary heart disease in three countries: A crosscultural comparison. International Joumal of Epidemiology, 21, 1081-1089. 
Syme, S.L. (1987). Coronary artery disease: A sociocultural perspective. Circulation, 76, 1112-1116.

Theorell, T. (1973). Psychosocial factors and myocardial infarction - why and how? Advances in Cardiology, 8, 117-131.

Theorell, T., De Faire, U., Schalling, D., Adamson, U., \& Askevold, F. (1979). Personality traits and psychophysiological reactions to a stressful interview in twins with varying degrees of coronary heart disease. Joumal of Psychosomatic Research, 23, 89-99.

Thiele, R., Simon, H., \& Thiele, G. (1985). Stressfaktoren bei Herzinfarktpatienten vor Eintritt des Herzinfarktes. Zeitschrift Gesamte Innere Medizin, 40, 483-488.

Verhagen, F., Nass, C., Appels, A., van Bastelaer, A., \& Winnubst, I. (1980). Cross-validation of the A/B typology in the Netherlands. Psychotherapy and Psychosomatics, 34, 178-186.

Vernick, L.J., Vernick, S.L., \& Kuller, L.H. (1984). Selection of neighborhood controls: logistics and fieldwork. Journal of Chronic Diseases, 37, 177-182.

Wacholder, S., Silverman, D.T., McLaughlin, J.K., \& Mandel, J.S. (1992). Selection of controls in case-control studies. II. Types of controls. American Joumal of Epidemiology, 135, 1029-1041.

Wardwell, W., Bahnson, C., \& Caron, H. (1963). Social and psychological factors in coronary heart disease. Jowrnal of Health and Social Behavior, 4, 154-165.

Watson, D., \& Pennebaker, J.W. (1989). Heath complaints, stress, and distress: Exploring the central role of negative affectivity. Psychological Review, 96, 234254.

Williams, R.B. (1987). Refining the type A hypothesis: emergence of the hostility complex. American Journal of Cardiology, 60, 27-32j.

Williams, R.B. (1994). Basic biological mechanisms. In A.W. Siegman \& T.W. Smith (Eds.), Anger, hostility, and the heart (pp. 117-125). Hillsdale, NJ: Lawrence Erlbaum Associates.

Williams, R.B., \& Barefoot, I.C. (1988). Coronary-prone behavior: The emerging role of the hostility complex. In B.K. Houston \& C.R. Snyder (Eds.), Type A behavior: Research, theory, and intervention (pp. 189-211). New York: Wiley.

Williams, R.B., Barefoot, J.C., Haney, T.L., Harrell, F.E., Blumenthal, J.A., Pryor, D.B., \& Peterson, B. (1988). Type A behavior and angiographically documented coronary atherosclerosis in a sample of 2,289 patients. Psychosomatic Medicine, $50,139-152$.

Williams, R.B., Barefoot, J.C., \& Shekelle, R.B. (1985). The health consequences of hostility. In M.A. Chesney \& R.H. Rosenman (Eds.), Anger and hostility in cardiovascular and behavioral disorders (pp. 173-185). Washington, DC: Hemisphere.

Williams, R.B., Haney, T.I., Lee, K.L., Kong, Y., Blumenthal, J., \& Whalen, R. (1980). Type A behavior, hostility, and coronary atherosclerosis. Psychosomatic Medicine, 42, 539-549. 
Wolf, S. (1969). Psychosocial forces in myocardial infarction and sudden death. Circulation, 40, 74-83.

Woodall, K.L., \& Matthews, K.A. (1989). Familial environment associated with Type A behaviors and psychophysiological responses to stress in children. Health Psychology, 8, 403-426.

Woodall, K.L., \& Matthews, K.A. (1993). Changes in and stability of hostile characteristics: Results from a 4-year longitudinal study of children. Journal of Personality and Social Psychology, 64, 491-499.

Wrzesniewski, K., Forgays, D.G., \& Bonaiuto, P. (1990). Measurement of the Type A behavior pattern in adolescents and young adults: Cross-cultural development of AATAB. Journal of Behavioral Medicine, 13, 111-135.

Zillmann, D. (1979). Hostility and aggression. New York: Wiley. 


\section{SUMMARY}

The present thesis investigates the role of two potential risk indicators of myocardial infarction (MI), viz. hostility and vital exhaustion (VE). Hostility is a multidimensional construct which can be divided into affective, behavioral, and cognitive components. In general, a hostile person is someone who has no trust in other people and views his fellow man as generally mean and selfish. Vital exhaustion is a state which is characterized by three central aspects: (1) unusual fatigue, (2) increased irritability, and (3) feelings of demoralization. To test the association between hostility, VE, and MI a case-control study was designed. The subjects in this case-control study were 249 men between 35 and 65 years of age from South Limburg, the southernmost region of the Netherlands. The cases were 81 consecutive patients who were hospitalized for a first documented MI. They were admitted to two regional hospitals in January 1990 and December 1990. The control group comprised 168 neighborhood controls living on the same street as cases or on an adjacent street at the time of the latter"s MI. Both cases and controls completed a set of questionnaires and were subsequently interviewed at home.

Chapter 1 presents an introduction to the concepts of hostillity and VE. This chapter begins with an overview of the association between hostility and health. The current interest in hostility is placed in a historical context and conceptual and assessment issues are discussed. Furthermore, an extensive review of research on hostility is included. Finally, the potential mechanisms underlying the relationship between hostility and health as well as the developmental aspects of hostility are described briefly. The next section deals with the mental precursors of MI. The origins of the concept of vital exhaustion (VE) are outlined and a summary is given of both prospective and cross-sectional evidence regarding the association with coronary heart disease (CHD). Chapter 1 concludes with an outline of the present thesis.

In Chapters 2 to 8 , the findings of the present study are presented. The most important results will be summarized below.

Hostility, as measured with the Cook-Medley Hostility (Ho) Scale, was found to be associated with an increased risk of first MI. However, the results indicated that this association was limited to people under 50. No relationship was observed in older subjects (Chapter 2).

Chapter 3 explores which dimensions of hostility are associated with increased risk of MI. Using the Buss-Durkee Hostility Inventory (BDHI), it was shown that experiential hostility (comprised of the BDHI subscales Resentment and Suspicion) was significantly associated with MI. 
Two studies examined the relationship between perceived parental rearing behavior and individual differences in hostility (Chapters 4 and 5). In a sample of 291 young adults (187 females and 104 males), highly hostile subjects were found to perceive more rejection and overprotection and less emotional warmth from both their father and their mother than low hostility subjects. In addition, perceived rejection (especially by the father) proved to be the strongest predictor of level of hostility in both sexes (Chapter 4). Inspection of the database of the case-control sample revealed a similar pattern of findings. In both cases and controls high hostility was related to perceived parental rejection and perceived lack of emotional warmth. Again, perceived rejection by the parents seemed to be the most strongly predictive of an individual's level of hostility (Chapter 5).

Chapter 6 reports on the development of an interview designed to measure the state of vital exhaustion (VE). This state can be assessed by means of the Maastricht Questionnaire (MQ). However, the MQ was observed to have some major shortcomings, especially for the selection of subjects for laboratory or clinical research. Therefore, an interview to measure VE was designed and compared with the MQ. It was found that the interview had more discriminating power than the MQ.

In Chapter 7, data on the psychometric qualities of the interview are presented. The interview was found to have good reliability and to discriminate significantly between cases and controls, thereby lending support to its validity, that is, the interview provided a valid assessment of the mental precursors of MI.

The results of a test of the association between hostility and VE are discussed in Chapter 8. VE appeared to be positively related to several dimensions of hostility. Furthermore, when hostility and VE were included simultaneously in a multivariate analysis, hostility lost its discriminating power, whereas VE remained significantly associated with MI. These results suggest a sequential relationship between hostility and VE, i.e. the risk for CHD conferred by hostility might be due to the fact that highly hostile people are at elevated risk of becoming exhausted.

Chapter 9 provides an overview of the main findings and a discussion of the implications for future research and possible intervention and prevention strategies. 


\section{SAMENVATTING}

Dit proefschrift beschrijft een onderzoek naar de rol van twee mogelijke risico indicatoren voor een myocard infarct (MD): vijandigheid en vitale uitputting (VU). Vijandigheid is een multidimensioneel concept dat kan worden onderverdeeld in 3 elementen: emotie, gedrag en cognitie. In het algemeen kan een vijandig individu worden getypeerd als iemand die geen vertrouwen heeft in de medemens en anderen beschouwt als gemeen en zelfzuchtig. Vitale uitputting is een toestand die wordt gekenmerkt door drie aspecten: ongewone vermoeidheid, toegenomen prikkelbaarheid en gevoelens van demoralisatie. Om de relatie tussen vijandigheid, VU en MI te onderzoeken werd een case-controle onderzoek opgezet. Deelnemers aan dit onderzoek waren 249 mannen uit Zuid-Limburg in de leeftijd van 35 tot 65 jatar. Cases waren 81 patienten die in het ziekenhuis waren opgenomen in verband met een eerste MI. Zij verbleven in twee regionale ziekenhuizen tussen januari 1990 en december 1990. De controle groep bestond uit 168 personen die in dezelfde of een aangrenzende straat woonachtig waren als de MI patiënten. Zowel MI patiënten als controles vulcen een aantal vragenlijsten in en werden vervolgens thuis geïnterviewd.

Hoofdstuk 1 biedt een inleiding tot de concepten van vijandigheid en VU. Dit hoofdstuk begint met een overzicht van de literatuur over de samenhang tussen vijandigheid en gezondheid. Verder worden mechanismen besproken die het verband tussen vijandigheid en gezondheid mogelijk verklaren en wordt er kort ingegaan op factoren die het ontstaan van vijandigheid tijdens de kinder - en jeugdjaren in de hand kunnen werken. De volgende paragraaf is gewijd aan de psychologische prodromale verschijnselen van het MI. De oorsprong van het concept vitale uitputting (VU) wordt beschreven en de bevindingen van prospectief en cross-sectioneel onderzoek naar de relatie tussen VU en hart- en vaatziekte (HVZ) worden samengevat. Hoofdstuk 1 wordt afgesloten met een overzicht van het onderhavige proefschrift.

In Hoofdstuk 2 tot en met 8 worden de resultaten wan het huidige onderzoek gepresenteerd. De belangrijkste bevindingen zullen in het hierna volgende worden samengevat.

Vijandigheid, zoals gemeten door de Cook-Medley Hostility schaal, bleek samen te hangen met een verhoogd risico voor een eerste MI. De resultaten lieten evenwe] zien dat deze associatie alleen waargenomen werd bij de groep mensen jonger dan 50 jaar. Bij oudere personen werd geen verband vastgesteld (Hoofdstuk 2).

Hoofdstuk 3 onderzoekt welke dimensies van vijandigheid verbonden zijn met een verhoogde kans op een MI. De dimensie "ervaren vijandigheid" (gemeten met be- 
hulp van de Buss-Durkee Hostility vragenlijst) bleek significant gerelateerd aan het MI.

Twee studies richten zich op de samenhang tussen waargenomen ouderlijke opvoedingsstijlen en individuele verschillen in vijandigheid (Hoofdstuk 4 en 5). Uit een steekproef bestaande uit jong volwassenen (187 vrouwen en 104 mannen) kwam naar voren dat vijandige personen meer verwerping en overbescherming en minder emotionele warmte van de kant van hun ouders rapporteerden dan niet vijandige personen. Verder bleek voor zowel mannen als vrouwen verwerping (in het bijzonder van cle vader) de meest krachtige voorspeller van de mate van vijandigtheid te zijn (Hoofdstuk 4). Onderzoek van de dataset van de case-controle steekproef bracht gelijksoortige resultaten aan het licht. Zowel voor cases als controles hing een hoge mate van vijandigheid samen met gerapporteerde verwerping en gebrek aan emotionele warmte. Verwerping bleek ook hier de mate van vijandigheid het beste te voorspellen (Hoofdstuk 5).

Hoofdstuk 6 doet verslag van de ontwikkeling van een interview om vitale uitputting (VU) te meten. Deze toestand kan worden vastgesteld met behulp van de Maastrichtse Vragenlijst (MV). Gebleken is echter dat de MV tekort schiet bij de selectie van proefpersonen voor laboratorium- of klinisch onderzoek. Derhalve werd een interview ontworpen om VU te meten. Dit interview werd vervolgens met de MV vergeleken. Het discriminerend vermogen wan het interview bleek groter dan dat van de MV.

In hoofdstuk 7 worden gegevens over de psychometrische eigenschappen van het interview gepresenteerd. Het interview bleek een betrouwbaar meetinstrument en discrimineerde tussen cases en controles. Deze laatste bevinding verleent steun aan de validiteit van het interview, dat wil zeggen: het interview maakt valide meting van de psychologische prodromale verschijnselen van het MI mogelijk.

Een onderzoek naar de relatie tussen vijandigheid en VU wordt beschreven in hoofdstuk 8 . VU bleek te correleren met verschillende dimensies van vijandigheid. Gelijktijdige opname van vijandigheid en VU in één multivariate analyse toonde aan dat vijandigheid niet langer discrimineerde tussen cases en controles. VU daarentegen behield haar discriminatief vermogen. Deze bevindingen suggereren een sequentiële relatie tussen vijandigheid en VU: de kans op $\mathrm{HVZ}$ verbonden aan vijandigheid kan mogelijk worden verklaard door het feit dat vijandige mensen een verhoogde kans hebben om uitgeput te raken.

Hoofdstuk 9, tenslotte, biedt een overzicht van de belangrijkste bevindingen alsmede een discussie met betrekking tot implicaties voor toekomstig onderzoek en mogelijke interventie- en preventiestrategieën. 


\section{APPENDIX}

\section{THE MAASTRICHT INTERVIEW FOR VITAL EXHAUSTION}

(in dutch)

Respondentnummer.

Naam.

Datum

Interviewer.

Instructie: Ik wil u enige vragen stellen hoe u zich de laatste tijd gevoeld hebt. Met de laatste tijd bedoel ik de laatste weken of maanden of misschien het laatste jaar. Tijdens thet gesprek kom ik daar nog op terug.

1. Voelt u zich de laatste tijd vaak moe?

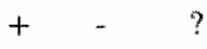

Hoelang voelt u zich al moe?

Is het erger geworden?

2. Voelt u zich de laatste tijd lusteloos?

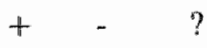

Hoelang hebt u dit gevoel al?

Is het erger geworden?

3. Voelt u zich de laatste tijd slap

$+\quad-\quad ?$

of zonder energie?

Hoelang hebt u dat gevoel al?

4. Bent u de laatste tijd prikkelbaarder dan vroeger?

$+\quad-\quad ?$

Wanneer is dat begonnen?

5. Bent u de laatste tijd moedeloos?

$+\quad-\quad$ ?

Warneer is dat begonnen?

6. Hebc $u$ het gevoel dat uw lichaam een batterij is

$+\quad-\quad ?$ waarvan de kracht of het vermogen aan het opraken is? 
7. Hebt $u$ het gevoel dat u de latatste tijd weinig + - ? presteert of minder in staat bent iets te presteren?

8. Wordt u de laatste tijd wel. eens wakker met een gevoel van uitputting en vermoeidheid?

9. Wordt U's nachts waak wakker? Zo ja, is dat voor u een probleem?

10. Hebt u de laatste tijd weleens het gevoel dat het allemaal wat teveel wordt?

1. H. Hebt $u$ het verlangen voelen opkomen om het bijltje erbij neer te leggen?

12. Is het zo, dat allerlei kleine dingen $u$ meer ergeren dan vroeger?

13. Bent $u$ opvliegender geworden?

14. Is het zo dat u de laatste tijd nergens zin meer in hebt, nergens meer toe komt?

15. Is de laatste tijd uw sexuele interesse, zin in vrijen, afgenomen?

16. Doet u er nu langer over om een moeilijk probleem te begrijpen?

17. Is het moeilijker geworden om u lang op een ding te kunnen concentreren?

18. Kunt u de laatste tijd tegen uw gewone werk als tegen een berg opzien?

19. Hebt u de laatste tijd het gevoel dat u in een impasse, in het slop zit?

20. Hebt u de laatste tijd weleens huilbuien of de neiging tot huilen?

21. Voelt u zich gedesillusioneerd of verslagen?

22. Hebt u de laatste tijd weleens een gevoel van hopeloosheid gehad? 
23. Denkt u de laatste tiju weleens:

$+\quad-\quad ?$

ik zou dood willen zijn?

24. Indien van toepassing.

U beschreef zich zelf in dit gesprek als iemand die moe/lusteloos/energieverlies/prikkelbaar/moedeloos was. Wanneer ik $U$ goed begrepen heb is dit ongeveer... maanden geleden begonnen.

Is dat juist?

Interviewer: controleer of alle vragen zijn gesteld en de antwoorden zijn omcirkeld.

25. Interview score vitale uitputting 



\section{LIST OF PUBLICATIONS}

Appels, A., Kop, W., Meesters, C., Markusse, R., Golombeck, B., \& Falger, P. (1994). Vital exhaustion and the acute coronary syndromes. In S. Maes, H. Leventhal, \& M. Johnston (Eds.), International Review of Healh Psychology, Volume 3 (pp. 65-95). New York: Wiley.

Appels, A., Meesters, C., \& Collijn, D. (1995). Sociaal-economische status en infarctrisico. De verklarende betekenis van hostiliteit en vitalle uitputting. Gedrag en Gezondheid, in press).

Appels, A., Meesters, C., Collijn, D., \& Schouten, E. (1995). Zijn psychosociale factoren vooral in de lagere sociaal-economische strata verbonden met gezondheid? (submitted).

Meesters, C.M.G. (1992). Boekbespreking van "Early detection of psychiatric disorders in children". (van Engeland, H.; Ed.). De Psycholoog, 27, 29.

Meesters, C.M.G. (1992). Hostiliteit en boosheid als risicofactoren voor hart- en vaatziekten. Psychoskoop, 3, 4-6.

Meesters, C., \& Appels, A. (1995). An interview to measure vital exhaustion, I. Development and comparison with the Maastricht Questionnaire. Psychology and Healih, in press.

Meesters, C., \& Appels, A. (1995). An interview to measure vital exhaustion. II. Reliability and validity of the interview and correlations of vital exhaustion with personality characteristics. Psychology and Health, in press.

Meesters, C., \& Appels, A. (1995). Hostility, vital exhaustion and myocardial infarction: Discriminating power and interrelationships. (submitted).

Meesters, C., \& Muris, P. (1995). The relationship between hostility and perceived parental rearing behaviour: A study of male myocardial infarction patienis and. healthy controls. (submitted).

Meesters, C.M.G., Muris, P., \& Backus, I. (1995). Dimensions of hostility and myocardial infarction in adult males. Journal of Psychosomatic Research, in press.

Meesters, C., Muris, P., Beuving, S., \& Schouten, E. (1995). Psychometric evaluation of the Aggression Questionnaire: A comment on Harris. (submitted).

Meesters, C., Muris, P., \& Esselink, T. (1995). Hostility and perceived parental rearing behaviour. Personality and Individual Differences, 18, 567-570.

Meesters, C.M.G., \& Smulders, I. (1992). Hostiliteit als risicofactor voor het hartinfarct: een case-control studie. Psychoskoop, 3, 154-158.

Meesters, C.M.G., \& Smulders, J. (1994). Hostility and myocardial infarction in men. Journal of Psychosomatic Research, 38, 727-734. 
Meesters, C., Sage, J. le, Muris, P., \& Mierlo, G. van. (1995). Slachtofferervaringen bij een RIAGG populatie. Gedrag en Gezondheid, in press.

Meijel-Moonen, M. van, \& Meesters, C. (1995). "Back to the future" - "Zurück in die Zukunft": Eine bessere Zukunft durch das Wiedererleben früherer Lebenserfahrungen. International Journal for Prenatal and Perinatal Psychology and Medicine, 7, 1-15.

Mendes de Leon, C.F., \& Meesters, C.M.G. (1991). Hostility and anger as risk factors of coronary heart disease. In A. Appels (Ed.), Behavioral observations in cardiovascular research (pp. 107-127). Amsterdam/Lisse: Swets \& Zeitlinger.

Muris, P., Bögels, S., van der Kamp, N., \& Meesters, C. (1995). Perception of parental rearing behaviour in clinically referred children: Correspondence between parent and child reports, and relationship to fearfulness and problem behaviour. (submitted).

Muris, P., Meesters, C., Merckelbach, H., \& Lomme, M. (1995). Knowledge of basic emotions in adolescent and adult individuals with autism. Psychological Reports, 76, 52-54.

Muris, P., Meesters, C., Ploumen, M., Bögels, S., van de Berg, M., Loman, F., \& Schouten, E. (1995). Samenhang tussen de Child Behavior Checklist (CBCL) en de DSM-classificatie. Kind en Adolescent, 16, 170-175.

Muris, P., Steerneman, P., Merckelbach, H., \& Meesters, C. (1995). The role of parental fearfulness and modeling in children's fear. Behaviour Research and Therapy, in press.

Steegmans, P.H.A., Hoes, A.W., Bak, A.A.A., Meesters, C.M.G., van der Does, E., \& Grobbee, D.E. (1995). Depression, hostility and impulsivity in middleaged men with low serum cholesterol levels. (submitted).

Wijers, M.G.P., Meesters, C.M.G., \& Gerards, F.M. (1992). Kindermishandeling, op school gesignaleerd. Maandblad Geestelijke volksgezondheid, 5, 522-532.

\section{Abstracts/Proceedings:}

Meesters, C.M.G., \& Simulders, J. (1992). Hostility as a risk factor of coronary heart disease. Proceedings of the Second International Congress of Behavioral Medicine, 1992 (Hamburg, Germany: 101).

Meesters, C., \& Appels, A. (1994). Hostility and vital exhaustion: Their association with coronary heart disease. Proceedings of the Third International Congress of Behavioral Medicine, 1994 (Amsterdam, The Netherlands: 84).

Meesters, C.M.G., \& Appels, A.P.W.M. (1995). An interview to measure vital exhaustion. Development, comparison with the Maastricht Questionnaire, and correlations with other psychosocial characteristics. Annals of Behavioral Medicine, 17, 127. 


\section{DANKWOORD}

Tot slot wil ik iedereen die op enigerlei wijze heeft bijgedragen aan de totstandkoming van dit proefschrift dank zeggen.

In de eerste plaats gaat mijn dank uit naar alle deelnemers aan het onderzoek, zowel hartinfarctpatienten als controles, die mij zonder uitzondering gastvrij hebben ontvangen. Zonder hun belangeloze medewerking zou dit proefschrift nooit zijn geschreven. Ook ben ik de medewerkers van de afdelingen cardiologie van het De Wever Ziekenhuis in Heerlen en het Academisch Ziekenhuis Maastricht erkentelijk voor het openstellen van hun afdeling voor de werving van proefpersonen. Met name noemen wil ik Theo Bergmans, die als hoofdverpleegkundige van afdeling Cardiologie 3 West van het De Wever Ziekenhuis altijd tijd wist vrij te maken en op zeer enthousiaste en betrokken wijze zelf actief patiënten stimuleerde om deel te nemen aan het onderzoek.

Mijn promotor, Prof. Dr. Ad Appels, wil ik bedanken voor het in mij gestelde vertrouwen gedurende de afgelopen jaren. Dat onze samenwerking vruchtbaar is geweest, moge blijken uit het feit dat onze namen boven een aantal gemeenschappelijke publikaties prijken.

Uiteraard op deze plaats ook een woord van dank aan de leden van de beoordelingscommissie: Prof. Dr. M.A. van den Hout (voorzitter), Dr. F.W.H.M. Bär, Dr. J. Diederiks, Prof. Dr. P.G. Heymans (Universiteit Utrecht) en Prof. Dr. J.W. van Ree.

Niet onvermeld mogen blijven de onmisbare inzet en steun op het vlak van proefpersoonwerving en het afnemen van interviews. In de eerste plaats is daar Isel van Noppen, die niet alleen een mooie prestatie heeft geleverd wat betreft de layout van dit boekje, maar bovenal in een eerdere fase van het onderzoek veel administratief werk heeft verricht. De blijmoedigheid waarmee zij bijna dagelijks (en dat bijna anderhalf jaar lang) het postcodeboek en vervolgens het telefoonboek van de regio Zuid-Limburg doorwroette, op zoek naar mogelijke kandidlaten voor het onderzoek, staat mij nog helder voor de geest. Voor de afname van een aantal interviews wil ik Winy Bax en Dorien Wolfs bedanken.

Een niet te onderschatten bijdrage aan het onderzoek is verder geleverd door een aantal studenten, die in het kader van hun onderzoeksstage een doctoralscriptie hebben geschreven: Jesca Smulders, Truus Esselink, Ingrid Backus, Irene Neissen, Marieke Hofman, bedankt voor jullie enthousiasme en plezierige samenwerking.

Ook wil ik Stella van Rijsoort en Natasja Hehanussa bedanken, die als studentassistenten ongeveer 250 op audioband opgenomen Type A interviews diverse malen hebben afgeluisterd en gecodeerd. Geen sinecure, gelet op de dikwijls aanwezige achtergrondruis in de vorm van fluitende kanaries, krijsende parkieten en 
hardop meedenkende familielleden van de geïnterviewde. Ook Aid Appels, die alle interviews ter bepaling van vital uitputting heeft beluisterd kan hierover meepraten.

Verder wil ik alle leden van divisie VIII "Psychosociale factoren van hart- en valziekten" en de collega's van de vakgroepen Medische Psychologie en Differentiêle en Experimentele Psychologie bedanken voor de prettige sfeer en de goede verstandhouding waarin ik altijd heb kunnen werken. Daarbij verdienen enkele collega's speciale vermelding. Allereerst Erik Schouten, van wie ik veel heb opgestoken op het terrein van de statistiek en de methodologie. Maar belangrijker nog was zijn functie als stabiel baken waarop men kan terugvallen als men er zelf niet meer uit dreigt te komen. Erik, bedankt voor alles. Ook van Hans Bosma en Jan de Jonge heb ik het een en ander opgestoken inzake statistische vraagstukken.

Peter Muris wil ik graag bedanken woor zijn stimulerende invloed tijdens de laatste fase van het onderzoek. Zijn aanstekelijke geestdrift heeft mij laten zien hoe leuk schrijven kan zijn en zijn gevleugelde woorden "quick and dirty studies" zijn inmiddels een begrip geworden.

Marijke van der Horst wil ik dank zeggen voor de zorgvuldige wijze waarop zij de engelse tekst heeft gecorrigeerd.

Onderwijs, specifieker gezegd het contact met studenten is voor mij altijd een bron van inspiratie geweest en heeft mij steeds veel voldoening geschonken en de energie gegeven om door te zetten op momenten dat ik het even niet zag zitten.

Tot slot wil ik Gemma, Vera en Astrid bedanken voor het geduld dat ze hebben weten op te brengen. Met name het laatste jaar ben ik voor hen minder beschikbaar geweest dan wenselijk zou zijn. Ik hoop dat zeer verbaasde uitroepen als "Papa, ga je ook mee wandelen? Moet je niet werken?" in de toekomst tot het verleden zullen gaan behoren. 


\section{CURRICULUM VITAE}

Cor Meesters werd op 26 november 1957 geboren te Sittard. Na het behalen van het diploma Gymnasium- $\alpha$ in 1976, studeerde hij psychologie aan de Katholieke Universiteit te Nijmegen. In 1983 studeerde hij af in de ontwikkelingspsychologie (Prof. Dr. F.J. Mönks), met als nevenrichtingen klinische psychologie en orthopedagogiek. Van 1983 tot 1984 was hij als docent ontwikkelingspsychologie verbonden aan de Katholieke Leergangen Tilburg. Vervolgens werkte hij van 1984 tot 1986 op free-lance basis voor de Hugo de Grootstichting (Prof. Dr. F.J. Mönks), waarbij hij belast was met psychodiagnostisch onderzoek en begeleiding van hoogbegaafde kinderen en hun ouders. In 1985 had hij een kortdurende aanstelling als toegevoegd docent bij de vakgroep ontwikkelingspsychologie van de Katholieke Universiteit te Nijmegen (Prof. Dr. F.J. Mönks / Prof. Dr. C.F.M. van Lieshout). Van januari 1986 tot maart 1987 was hij aangesteld als klinisch (ontwikkelings)psycholoog bij de afdeling Medische Psychologie (Prof. Dr. P.B. Bierkens) en voor de patiëntenzorg gedetacheerd werkzaam bij het Interdisciplinair Kinder Neurologisch Centrum (IKNC; Prof. Dr. F. Gabreëls) van het Academisch Ziekenhuis Nijmegen. Sedert maart 1987 is hij in dienst van de faculteit der Gezondheidswetenschappen van de Rijksuniversiteit Limburg en als universitair docent ontwikkelingspsychologie werkzaam bij de vakgroep Medische Psychologie (voorzitter: Prof. Dr. A.P.W.M. Appels). Aandachtsgebieden binnen het onderwijs zijn ontwikkelingspsychologie, kinder- en jeugdpsychopathologie en psychodiagnostiek. Voorts maakt hij deel uit van CARIM (Cardiovascular Research Institute Maastricht) en verricht hij wetenschappelijk onderzoek binnen divisie VIII "Psychosociale factoren van hart- en vaatziekten" (divisieleider: Prof. Dr. A.W.P.M. Appels). 